\title{
Lattice-Valued Topological Systems as a Framework for Lattice-Valued Formal Concept Analysis
}

\author{
Sergey A. Solovyov ${ }^{1,2}$ \\ ${ }^{1}$ Department of Mathematics and Statistics, Faculty of Science, Masaryk University, Kotlarska 2, 61137 Brno, Czech Republic \\ ${ }^{2}$ Institute of Mathematics and Computer Science, University of Latvia, Raina bulvaris 29, Riga 1459, Latvia \\ Correspondence should be addressed to Sergey A. Solovyov; solovjovs@math.muni.cz
}

Received 26 August 2012; Accepted 9 November 2012

Academic Editor: Alfred Peris

Copyright (C) 2013 Sergey A. Solovyov. This is an open access article distributed under the Creative Commons Attribution License, which permits unrestricted use, distribution, and reproduction in any medium, provided the original work is properly cited.

\begin{abstract}
Recently, Denniston, Melton, and Rodabaugh presented a new categorical outlook on a certain lattice-valued extension of Formal Concept Analysis (FCA) of Ganter and Wille; their outlook was based on the notion of lattice-valued interchange system and a category of Galois connections. This paper extends the approach of Denniston et al. clarifying the relationships between Chu spaces of Pratt, many-valued formal contexts of FCA, lattice-valued interchange systems, and Galois connections.
\end{abstract}

\section{Introduction}

This paper considers a particular application of the theory of variety-based topological systems, introduced in [1] as a generalization of topological systems of Vickers [2], which in turn provide a common framework for both topological spaces and their underlying algebraic structures frames (also called locales), thereby allowing researchers to switch freely between the spatial and localic viewpoints. Vickers' concept, which was motivated by the theory of crisp topology, has recently drawn the attention of fuzzy topologists, who have incorporated the notion in their topic of study. The first attempt in this respect was done by Denniston and Rodabaugh [3], who considered various functorial relationships between topological systems of Vickers and latticevalued topological spaces of Rodabaugh [4]. Soon afterwards, the study of Guido [5, 6] followed; he used topological systems in order to construct a functor from the category of $L$-topological spaces to the category of crisp topological spaces, thereby providing a common framework for various approaches to the hypergraph functor of the fuzzy community (see, e.g., [7]).

It soon appeared though that the translation of the most important tools of the theory of topological systems (e.g., system spatialization and localification procedures) into the language of lattice-valued topology required a suitable fuzzification of the former concept, which was accomplished successfully by Denniston et al. in [8]. The authors brought their theory into maturity in [9], where they considered its applications to both lattice-valued variable-basis topology of Rodabaugh [4] and ( $L, M)$-fuzzy topology of Kubiak and Šostak [10]. Later on, they introduced a particular instance of their concept called interchange system [11], which was motivated by certain aspects of program semantics (the socalled predicate transformers) initiated by Dijkstra [12]. It should be noted, however, that an interchange system is a particular instance of the well-known concept of Chu space in the sense of Pratt [13] (the original notion goes back to Barr [14]). Moreover, the interchange system morphisms of [11] are precisely the Chu space morphisms (called Chu transforms) of [13]. (In [15], Denniston et al. use the terms transformer system and transformer morphism.) The underlying motivations of interchange systems and Chu spaces are quite different, which is reflected well enough in both their names and their respective theories.

Parallel to the previously mentioned developments, we introduced the concept of variety-based topological system [1], which not only extended the setting of Denniston et al., but also included the case of state property systems of Aerts [16-18] (introduced as the basic mathematical structure in the Geneva-Brussels approach to foundations of physics), considered in [19] in full detail, and we brought the functor of 
Guido to a new level [20] (e.g., made it variable-basis in the sense of Rodabaugh [4], as well as extended the machinery of Höhle [7], to construct its right adjoint functor). Moreover, in [21], we provided a thorough categorical extension of the system spatialization procedure to the variety-based setting, the respective localification procedure being elaborated in [22]. Additionally, certain aspects of the new theory bearing links to bitopological spaces of Kelly [23] as well as to noncommutative topology of Mulvey and Pelletier [24, 25] were treated extensively in $[26,27]$, respectively.

Seeing the fruitfulness of their newly introduced notions, both Denniston et al. and the author of this paper independently turned their attention to possible applications of the arising system framework to the areas beyond topology. In particular, in [28], we started the theory of lattice-valued soft universal algebra, whereas Denniston et al. presented in $[29,30]$ a challenging categorical outlook on a certain lattice-valued extension of Formal Concept Analysis (FCA) of Ganter and Wille [31]. Similar to the case of lattice-valued topological systems, their research motivated us to take a deeper look into the proposed topic of lattice-valued FCA, thereby streamlining the motivating approach of Denniston et al. and also extending some of their results.

Modifying slightly the definition of the category of Galois connections of McDill et al. [32], Denniston et al. [29] consider its relationships to the already mentioned category of interchange systems, the objects of both categories being essentially formal contexts of FCA. The main result is a categorical embedding of each category into its counterpart, the ultimate conclusion though amounting to the categories in question being rather different; that is, the two viewpoints on FCA are not categorically isomorphic. The rest of the paper provides a lattice-valued, fixed-basis extension of the crisp achievements, based on the lattice-valued FCA of Bêlohlávek [33]. Moreover, towards the end of the paper, with the help of the constructed embeddings, the authors compare the categories of (lattice-valued) formal contexts and (lattice-valued) topological systems inside the category of (lattice-valued) interchange systems, making a significant (and rather striking) metamathematical conclusion on their being disjoint.

The just mentioned approach of Denniston et al. can be extended, taking into consideration the following facts. Firstly, it never gives much attention to the links between interchange systems and Chu spaces, thereby making no use of the well-developed machinery of the latter. Secondly, while pursuing their approach to many-valued FCA, the authors never mention the already existing many-valued formal contexts of Ganter and Wille [31], which (being similar to Chu spaces) essentially extend their own. Thirdly, being categorically oriented, the paper introduces two types of formal context morphisms, in terms of Chu transforms and Galois connections, respectively, but misses the wellestablished context morphisms of FCA, which are different from those of the paper. Lastly (and most important), turning to fuzzification of their notions, the authors concentrate on the fixed-basis lattice-valued approach, notwithstanding that the more promising variable-basis setting (initiated by one of them) has already gained its popularity in the fuzzy community, currently being a kind of de facto standard for fuzzy structures.

The main purposes of this paper are to extend the results of Denniston et al. and to clarify the relationships between Chu spaces, many-valued formal contexts of FCA, latticevalued interchange systems, and Galois connections. In particular, we introduce three categories (each of them having a twofold modification, arising from its respective variablebasis), whose objects are lattice-valued versions of formal contexts of FCA, but whose morphisms are quite different, each instance reflecting the approach of Pratt, Ganter and Wille, and Denniston et al., respectively. Following the results of [29], we embed (nonfully) each of the categories into its two counterparts, showing, however, that the constructed embeddings fail to provide a categorical isomorphism (even an adjoint situation) between any two of the presented three approaches. As a consequence, we obtain three different categorical outlooks on FCA, which are done in the variable-basis lattice-valued framework of [4]. Moreover, the respective underlying lattices of the fuzzified formal contexts are quantales $[34,35]$ instead of complete residuated lattices of Denniston et al.; that is, the heavy assumption on commutativity of the quantale multiplication is dropped. In particular, we show lattice-valued generalizations of formal concept, preconcept, and protoconcept of [36], which are based on an arbitrary (namely, non-commutative) quantale (actually, an algebra, having a quantale as its reduct).

Similar to Denniston et al., we develop the required machinery of quantale-valued Galois connections, which extends that of Erné et al. [37] and which is motivated by the commutative approach of fuzzy Galois connections of Bêlohlávek [38]. For example, we provide yet another generalization to the quantale setting of the well-known fact that every order-reversing Galois connection between powersets arises as a pair of Birkhoff operators with respect to a binary relation [39]. The generalization though appears to be somewhat truncated, in the sense that while latticevalued binary relations still give rise to order-reversing Galois connections, the converse way is not always available $([38,40]$ do restore the full result even in the lattice-valued case, but rely on the more demanding notion of fuzzy Galois connection). Moreover, we define a category of order-preserving Galois connections as a certain subcategory of the category of Chu spaces, in which (unlike McDill et al. and Denniston et al.) Galois connections play the role of morphisms rather than objects, thereby providing particular instances of Chu transforms. We fail to provide a similar machinery for the order-reversing case.

At the end of the paper, we will notice that the approach to FCA through order-reversing Galois connections taken up by Denniston et al. does not suit exactly the underlying ideas of FCA of Ganter and Wille, which are formulated in terms of the incidence relations of formal contexts. The main sticking point here is the previously mentioned fact that the important crisp case one-to-one correspondence between order-reversing Galois connections on powersets and pairs of Birkhoff operators breaks down in the latticevalued case (quantales). As a consequence, the incidence relation viewpoint provides one with a Galois connection, 
whereas the latter alone lacks the strength of bringing a respective incidence relation in play (cf. Problems 1 and 2).

The paper is based on both category theory and universal algebra, relying more on the former. The necessary categorical background can be found in [41-43]. For algebraic notions, the reader is referred to $[34,35,44,45]$. Although we tried to make the paper as much self-contained as possible, it is expected from the reader to be acquainted with basic concepts of category theory, that is, those of category and functor.

\section{Algebraic Preliminaries}

For convenience of the reader, we begin with those algebraic preliminaries, which are crucial for the understanding of the results of the paper. Experienced researchers may skip this section, consulting it for the notations of the author only.

2.1. Varieties of Algebras. An important foundational aspect of the paper is the abstract algebraic structure (called algebra, for short) which is a set, equipped with a family of operations, satisfying certain identities. The theory of universal algebra calls a class of finitary algebras (induced by a set of finitary operations), which is closed under the formation of homomorphic images, subalgebras, and direct products, a variety. In this paper, we extend the notion of varieties to include infinitary algebraic theories. Our motivation includes ideas of [45-47].

Definition 1. Let $\boldsymbol{\Omega}=\left(n_{\lambda}\right)_{\lambda \in \Lambda}$ be a (possibly, proper or empty) class of cardinal numbers. An $\Omega$-algebra is a pair $\left(A,\left(\omega_{\lambda}^{A}\right)_{\lambda \in \Lambda}\right)$, which comprises a set $A$ and a family of maps $A^{n_{\lambda}} \stackrel{\omega_{\lambda}^{A}}{\longrightarrow}$ $A$ ( $n_{\lambda}$-ary primitive operations on $A$ ). An $\Omega$-homomorphism $\left(A_{1},\left(\omega_{\lambda}^{A_{1}}\right)_{\lambda \in \Lambda}\right) \stackrel{\varphi}{\rightarrow}\left(A_{2},\left(\omega_{\lambda}^{A_{2}}\right)_{\lambda \in \Lambda}\right)$ is a map $A_{1} \stackrel{\varphi}{\rightarrow} A_{2}$ such that the diagram

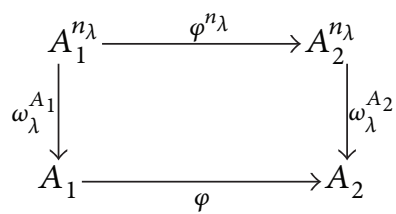

commutes for every $\lambda \in \Lambda$. $\operatorname{Alg}(\boldsymbol{\Omega})$ is the construct of $\boldsymbol{\Omega}$ algebras and $\boldsymbol{\Omega}$-homomorphisms.

Every concrete category of this paper has an underlying functor $|-|$ to the respective ground category (e.g., the category Set of sets and maps), the latter mentioned explicitly in each case.

Definition 2. Let $\mathscr{M}$ (resp., $\mathscr{E}$ ) be the class of $\boldsymbol{\Omega}$ homomorphisms with injective (resp., surjective) underlying maps. A variety of $\boldsymbol{\Omega}$-algebras are a full subcategory of $\operatorname{Alg}(\Omega)$, closed under the formation of products, $\mathscr{M}$ subobjects (subalgebras), and $\mathscr{E}$-quotients (homomorphic images). The objects (resp., morphisms) of a variety are called algebras (resp., homomorphisms).
Definition 3. Given a variety $\mathbf{A}$, a reduct of $\mathbf{A}$ is a pair $(\|-\|, \mathbf{B})$, where $\mathbf{B}$ is a variety such that $\boldsymbol{\Omega}_{\mathbf{B}} \subseteq \boldsymbol{\Omega}_{\mathbf{A}}$, and $\mathbf{A} \stackrel{\|-\|}{\longrightarrow} \mathbf{B}$ is a concrete functor. The pair $(\mathbf{A},\|-\|)$ is called then an extension of $\mathbf{B}$.

The following definitions recall several well-known varieties $[34,35,48,49]$, thereby providing the background for the subsequent developments of this paper.

Definition 4. CSLat $(\Xi)$ is the variety of $\Xi$-semilattices, that is, partially ordered sets (posets), which have arbitrary $\Xi \in$ $\{\bigwedge, \bigvee\}$ (meets and joins, resp.).

Given a $\Xi$-semilattice $A$, we have both $\bigwedge$ and $\bigvee$ (however, only one of them is respected by $\Xi$-semilattice homomorphisms) and denote its largest (resp., smallest) element $\mathrm{T}_{A}$ (resp., $\left.\perp_{A}\right)$. Given a $\bigvee$-semilattice $A$, we denote $2 A$ ? its respective $\bigwedge$-semilattice and vice versa. The standard result of [50, Sections $0-3]$ says that every $\bigvee$-semilattice homomorphism $A_{1} \stackrel{\varphi}{\rightarrow} A_{2}$ has the upper adjoint map $\left|A_{2}\right| \stackrel{\varphi^{\vdash}}{\rightarrow}\left|A_{1}\right|$, which is characterized uniquely by the condition $\varphi\left(a_{1}\right) \leqslant a_{2}$ iff $a_{1} \leqslant \varphi^{\vdash}\left(a_{2}\right)$ for every $a_{1} \in$ $A_{1}$ and every $a_{2} \in A_{2}$, which, for order-preserving maps only, is equivalent to the properties $\varphi \circ \varphi^{\vdash} \leqslant 1_{A_{2}}$ and $1_{A_{1}} \leqslant \varphi^{\vdash} \circ \varphi$ (given an algebra $A, 1_{A}$ denotes the identity map on $A)$. The explicit formula for the map is given by $\varphi^{\vdash}\left(a_{2}\right)=\bigvee\left\{a_{1} \in A_{1} \mid \varphi\left(a_{1}\right) \leqslant a_{2}\right\}$, whereas the map itself is easily shown to be $\bigwedge$-preserving. The dual description of the lower adjoint map (based on a $\bigwedge$-semilattice homomorphism $A_{1} \stackrel{\varphi}{\rightarrow} \quad A_{2}$ and denoted by $\left|A_{2}\right| \stackrel{\varphi^{-1}}{\longrightarrow}$ $\left|A_{1}\right|$ ) is left to the reader (see also Section 2.3 on Galois connections).

Definition 5. Quant is the variety of quantales, that is, triples $(Q, V, \otimes)$ where

(1) $(Q, \bigvee)$ is a $\bigvee$-semilattice;

(2) $(Q, \otimes)$ is a semigroup; that is, $q_{1} \otimes\left(q_{2} \otimes q_{3}\right)=\left(q_{1} \otimes\right.$ $\left.q_{2}\right) \otimes q_{3}$ for every $q_{1}, q_{2}, q_{3} \in Q$;

(3) $\otimes$ distributes across $\bigvee$ from both sides; that is, $q \otimes$ $(\bigvee S)=\bigvee_{s \in S}(q \otimes s)$ and $(\bigvee S) \otimes q=\bigvee_{s \in S}(s \otimes q)$ for every $q \in Q$ and every $S \subseteq Q$.

Every quantale $Q$ has two residuations, which are induced by its binary operation $\otimes$ (called multiplication) and which are defined by $q_{1} \rightarrow{ }_{l} q_{2}=\bigvee\left\{q \in Q \mid q \otimes q_{1} \leqslant q_{2}\right\}$ and $q_{1} \rightarrow{ }_{r} q_{2}=\bigvee\left\{q \in Q \mid q_{1} \otimes q \leqslant q_{2}\right\}$ (notice that "l" (resp., " $r$ ") stands for "multiply on the left" (resp., "right")), respectively, providing a single residuation $\rightarrow$. in case of a commutative multiplication (resulting in complete residuated lattices of Denniston et al. [29]). These operations have the standard properties of poset adjunctions [50, Sections 0-3] or (orderpreserving) Galois connections [37] (see Section 2.3 of this paper), for example, $q_{1} \leqslant q_{2} \rightarrow{ }_{l} q_{3}$ iff $q_{1} \otimes q_{2} \leqslant q_{3}$ iff $q_{2} \leqslant q_{1} \rightarrow{ }_{r} q_{3}$. For convenience of the reader, the following theorem recalls some of their other features, which will be heavily used throughout the paper. 
Theorem 6. The residuations $\cdot \rightarrow_{l}$ and $\cdot \rightarrow_{r}$. of a quantale $Q$ have the following properties.

(1) $q \rightarrow_{l}(\bigwedge S)=\bigwedge_{s \in S}\left(q \rightarrow_{l} s\right)$ and $q \rightarrow_{r}(\bigwedge S)=$ $\bigwedge_{s \in S}\left(q \rightarrow{ }_{r} s\right)$ for every $q \in Q$ and every $S \subseteq Q$. In particular, $q \rightarrow{ }_{l} \mathrm{~T}_{\mathrm{Q}}=\mathrm{T}_{\mathrm{Q}}$ and $q \rightarrow{ }_{r} \mathrm{~T}_{\mathrm{Q}}=\mathrm{T}_{\mathrm{Q}}$ for every $q \in Q$.

(2) $(\bigvee S) \rightarrow_{l} q=\bigwedge_{s \in S}\left(s \rightarrow_{l} q\right)$ and $(\bigvee S) \rightarrow_{r} q=$ $\bigwedge_{s \in S}\left(s \rightarrow{ }_{r} q\right)$ for every $q \in Q$ and every $S \subseteq Q$. In particular, $\perp_{\mathrm{Q}} \rightarrow_{l} q=\mathrm{T}_{\mathrm{Q}}$ and $\perp_{\mathrm{Q}} \rightarrow_{r} q=\mathrm{T}_{\mathrm{Q}}$ for every $q \in Q$.

(3) $q_{1} \leqslant\left(q_{1} \rightarrow{ }_{l} q_{2}\right) \rightarrow_{r} q_{2}$ and $q_{1} \leqslant\left(q_{1} \rightarrow{ }_{r} q_{2}\right) \rightarrow_{l} q_{2}$ for every $q_{1}, q_{2} \in Q$.

(4) $\left(q_{1} \otimes q_{2}\right) \rightarrow_{l} q_{3}=q_{1} \rightarrow_{l}\left(q_{2} \rightarrow{ }_{l} q_{3}\right)$ and $\left(q_{1} \otimes\right.$ $\left.q_{2}\right) \rightarrow_{r} q_{3}=q_{2} \rightarrow_{r}\left(q_{1} \rightarrow_{r} q_{3}\right)$ for every $q_{1}, q_{2}, q_{3} \in$ $Q$.

Proof. To give the flavor of the employed machinery, we show the proofs of some of the claims of the items.

Ad (3). $q_{1} \rightarrow_{l} q_{2} \leqslant q_{1} \rightarrow_{l} q_{2}$ implies $\left(q_{1} \rightarrow_{l} q_{2}\right) \otimes$ $q_{1} \leqslant q_{2}$ implies $q_{1} \leqslant\left(q_{1} \rightarrow{ }_{l} q_{2}\right) \rightarrow_{r} q_{2}$.

Ad (4). Given $q \in Q, q \leqslant\left(q_{1} \otimes q_{2}\right) \rightarrow_{l} q_{3}$ iff $q \otimes\left(q_{1} \otimes q_{2}\right) \leqslant q_{3}$ iff $q \otimes q_{1} \leqslant q_{2} \rightarrow_{l} q_{3}$ iff $q \leqslant$ $q_{1} \rightarrow_{l}\left(q_{2} \rightarrow_{l} q_{3}\right)$.

Moreover, $q \leqslant\left(q_{1} \otimes q_{2}\right) \rightarrow_{r} q_{3}$ iff $\left(q_{1} \otimes q_{2}\right) \otimes q \leqslant q_{3}$ iff $q_{2} \otimes q \leqslant q_{1} \rightarrow_{r} q_{3}$ iff $q \leqslant q_{2} \rightarrow_{r}\left(q_{1} \rightarrow_{r} q_{3}\right)$.

In the subsequent developments, we will consider some special types of quantales mentioned in the following.

Definition 7. CQuant is the variety of commutative quantales, which are quantales $Q$ with commutative multiplication; that is, $q_{1} \otimes q_{2}=q_{2} \otimes q_{1}$ for every $q_{1}, q_{2} \in Q$.

Definition 8. UQuant is the variety of unital quantales, which are quantales $Q$ having an element $1_{Q}$ such that the triple $\left(\mathrm{Q}, \otimes, 1_{\mathrm{Q}}\right)$ is a monoid; that is, $q \otimes 1_{\mathrm{Q}}=q=1_{\mathrm{Q}} \otimes q$ for every $q \in Q$.

Definition 9. SRSQuant (resp., SLSQuant) is the variety of strictly right-sided (resp., left-sided) quantales, which are quantales $Q$ such that $q \otimes T_{Q}=q\left(\right.$ resp., $\left.T_{Q} \otimes q=q\right)$ for every $q \in Q$.STSQuant is the variety of strictly two-sided quantales, that is, unital quantales whose unit is the top element.

Definition 10. Frm is the variety of frames, that is, strictly two-sided quantales, in which multiplication is the meet operation.

Definition 11. CBAlg is the variety of complete Boolean algebras, that is, frames $A$, in which $\Lambda$ are considered as primitive operations (cf. Definition 1) and which additionally are equipped with the complement operation $(-)^{\prime}$, assigning to every $a \in A$ an element $a^{\prime} \in A$ such that $a \wedge a^{\prime}=\perp_{A}$ and $a \vee a^{\prime}=\mathrm{T}_{A}$.

The varieties CBAlg, Frm, STSQuant, UQuant, and $\operatorname{CSLat}(\bigvee)$ provide a sequence of variety reducts in the sense of Definition 3. Additionally, Frm is an extension of CQuant.
The notations related to varieties in this paper follow the category-theoretic pattern (which is different from the respective one of the fuzzy community). From now on, varieties are denoted by $\mathbf{A}$ or $\mathbf{B}$, whereas those which extend the variety $\operatorname{CSLat}(\Xi)$ of $\Xi$-semilattices with $\Xi \in\{\bigvee, \Lambda\}$ will be distinguished with the notation $\mathbf{L}$. $\mathbf{S}$ will stand for either subcategories of varieties or the subcategories of their dual categories. The categorical dual of a variety $\mathbf{A}$ is denoted by $\mathbf{A}^{o p}$. However, the dual of Frm uses the already accepted notation Loc [48]. Given a homomorphism $\varphi$, the corresponding morphism of the dual category is denoted by $\varphi^{o p}$ and vice versa. Every algebra $A$ of a variety $\mathbf{A}$ gives rise to the subcategory $\mathbf{S}_{A}$ of either $\mathbf{A}$ or $\mathbf{A}^{o p}$, whose only morphism is the identity map $A \stackrel{1_{A}}{\longrightarrow} A$.

2.2. Powerset Operators. One of the main tools in the subsequent developments of the paper will be generalized versions of the so-called forward and backward powerset operators. More precisely, given a map $X_{1} \stackrel{f}{\rightarrow} X_{2}$, there exists the forward (resp., backward) powerset operator $\mathscr{P}\left(X_{1}\right) \stackrel{f^{\rightarrow}}{\longrightarrow}$ $\mathscr{P}\left(X_{2}\right)$ (resp., $\mathscr{P}\left(X_{2}\right) \stackrel{f^{\leftarrow}}{\longrightarrow} \mathscr{P}\left(X_{1}\right)$ ) defined by $f^{\rightarrow}(S)=$ $\{f(s) \mid s \in S\}$ (resp., $f^{\leftarrow}(T)=\left\{x \in X_{1} \mid f(x) \in T\right\}$ ). The two operators admit functorial extensions to varieties of algebras. (For a thorough treatment of the powerset operator theory, the reader is referred to the articles of Rodabaugh [49, 51-53], dealing with the lattice-valued case, or to the papers of the author himself $[21,54]$, dealing with a more general varietybased approach.)

To begin with, notice that given an algebra $A$ of a variety A and a set $X$, there exists the A-powerset of $X$, namely, the set $A^{X}$ of all maps $X \stackrel{\alpha}{\rightarrow} A$. Equipped with the pointwise algebraic structure involving that of $A, A^{X}$ provides an algebra of $\mathbf{A}$. In the paper, we will often use specific elements of this powerset algebra. More precisely, every $a \in A$ gives rise to the constant map $X \stackrel{a}{\rightarrow} A$ with the value $a$. Moreover, if $\mathbf{A}$ extends the variety $\operatorname{CSLat}(\bigvee)$ of $\bigvee$-semilattices, then every $S \subseteq X$ and every $a \in A$ give rise to the map $X \stackrel{\chi_{s}^{a}}{\longrightarrow} A(a$ characteristic map of $S$ ), which is defined by

$$
\chi_{S}^{a}(x)= \begin{cases}a, & x \in S \\ \perp_{A}, & \text { otherwise. }\end{cases}
$$

Of particular importance will be the characteristic maps of singletons, that is, $\chi_{\{x\}}^{\top_{A}}$ or $\chi_{\{x\}}^{1_{A}}$, the latter case assuming additionally that $\mathbf{A}$ extends the variety $\mathbf{U Q u a n t}$ of unital quantales.

Coming back to powerset operators, the following extension of the forward one has already appeared in a more general way in [28], being different from the respective lattice-valued approaches of Rodabaugh. 
Theorem 12. Given a variety $\mathrm{L}$, which extends $\operatorname{CSLat}(\bigvee)$, every subcategory $\mathbf{S}$ of $\mathbf{L}$ provides the functor $\mathbf{S e t} \times$ $\mathbf{S} \stackrel{(-) \rightarrow}{\longrightarrow} \operatorname{CSLat}(\bigvee)$, which is defined by $\left(\left(X_{1}, L_{1}\right) \stackrel{(f, \varphi)}{\longrightarrow}\right.$ $\left.\left(X_{2}, L_{2}\right)\right) \rightarrow=\left\|L_{1}^{X_{1}}\right\| \stackrel{(f, \varphi) \rightarrow}{\longrightarrow}\left\|L_{2}^{X_{2}}\right\|$, where $((f, \varphi) \rightarrow(\alpha))\left(x_{2}\right)=$ $\varphi\left(\bigvee_{f\left(x_{1}\right)=x_{2}} \alpha\left(x_{1}\right)\right)$.

Proof. To show that $\left\|L_{1}^{X_{1}}\right\| \stackrel{(f, \varphi)^{\rightarrow}}{\longrightarrow}\left\|L_{2}^{X_{2}}\right\|$ is $V$ preserving, notice that given $S \subseteq L_{1}^{X_{1}}$, for every $x_{2} \in X_{2},((f, \varphi) \rightarrow(\bigvee S))\left(x_{2}\right)=\varphi\left(\bigvee_{f\left(x_{1}\right)=x_{2}}(\bigvee S)\left(x_{1}\right)\right)=$ $\varphi\left(\bigvee_{f\left(x_{1}\right)=x_{2}} \bigvee_{s \in S} s\left(x_{1}\right)\right)=\varphi\left(\bigvee_{s \in S} \bigvee_{f\left(x_{1}\right)=x_{2}} s\left(x_{1}\right)\right)=$ $\bigvee_{s \in S} \varphi\left(\bigvee_{f\left(x_{1}\right)=x_{2}} s\left(x_{1}\right)\right)=\bigvee_{s \in S}((f, \varphi) \rightarrow(s))\left(x_{2}\right)=$ $\left(\bigvee_{s \in S}(f, \varphi) \rightarrow(s)\right)\left(x_{2}\right)$.

To show that the functor preserves composition, notice that given Set $\times \mathbf{S}$-morphisms $\left(X_{1}, L_{1}\right) \stackrel{(f, \varphi)}{\longrightarrow}$ $\left(X_{2}, L_{2}\right)$ and $\left(X_{2}, L_{2}\right) \stackrel{\left(f^{\prime}, \varphi^{\prime}\right)}{\longrightarrow}\left(X_{3}, L_{3}\right)$, for every $\alpha \in L_{1}^{X_{1}}$ and every $x_{3} \in X_{3},\left(\left(f^{\prime}, \varphi^{\prime}\right) \rightarrow\right.$ 。 $(f, \varphi) \rightarrow(\alpha))\left(x_{3}\right)=\varphi^{\prime}\left(\bigvee_{f^{\prime}\left(x_{2}\right)=x_{3}}((f, \varphi) \rightarrow(\alpha))\left(x_{2}\right)\right)=$ $\varphi^{\prime}\left(\bigvee_{f^{\prime}\left(x_{2}\right)=x_{3}} \varphi\left(\bigvee_{f\left(x_{1}\right)=x_{2}} \alpha\left(x_{1}\right)\right)\right)=\varphi^{\prime} \circ \varphi\left(\bigvee_{f^{\prime} \circ f\left(x_{1}\right)=x_{3}} \alpha\left(x_{1}\right)\right)=$ $\left(\left(f^{\prime} \circ f, \varphi^{\prime} \circ \varphi\right) \rightarrow(\alpha)\right)\left(x_{3}\right)=\left(\left(\left(f^{\prime}, \varphi^{\prime}\right) \circ(f, \varphi)\right) \rightarrow(\alpha)\right)\left(x_{3}\right)$.

The case $\mathbf{S}=\mathbf{S}_{L}$ is denoted by $(-)_{L}$ and is called a (variety-based) fixed-basis approach, whereas all other cases are subsumed under (variety-based) variable-basis approach. Similar notations and naming conventions are applicable to all other variety-based extensions of powerset operators introduced in this subsection and, for the sake of brevity, will not be mentioned explicitly.

There is another extension of the forward powerset operator, which uses the idea of Rodabaugh [52] (the reader is advised to recall the notation for lower and upper adjoint maps from the previous subsection). Before the actual result, however, some words are due to our employed powerset operator notations. In all cases, which are direct analogues of the classical powerset operators of the fuzzy community (e.g., those of Rodabaugh [51] and his followers), we use the solid arrow notation, that is, “( $(-)^{\leftarrow ”}$ ( (resp., “( $(-) \rightarrow$ ”) for backward (resp., forward) powerset operator. In the cases, which are not well known to the researchers, we use dashed arrow notation, that is, "(-) $\left.)^{(-\cdots) " ~(r e s p ., ~ "(-) ~}\right)^{-\cdots ”}$ ”) for backward (resp., forward) powerset operator, possibly, adding some new symbols (e.g., " - " or " $\vdash$ ") to underline the involvement of upper or lower adjoint maps in the definition of the operator in question (e.g., $(-)^{-\cdots)}$ or $(-)^{\vdash \cdots)}$ ) as in the following theorem.

\section{Theorem 13.}

(1) Given a variety $\mathbf{L}$, which extends $\mathbf{C S L a t}(\bigwedge)$, every subcategory $\mathbf{S}$ of $\mathbf{L}^{\text {op }}$ gives rise to the functor $\mathbf{S e t} \times \mathbf{S} \stackrel{(-)^{+-}}{\longrightarrow}$ $\operatorname{CSLat}(\bigvee)$ defined by $\left(\left(X_{1}, L_{1}\right) \stackrel{(f, \varphi)}{\longrightarrow}\left(X_{2}, L_{2}\right)\right)^{-\cdots \rightarrow}=$ $2 L_{1}^{X_{1}} \prec \stackrel{(f, \varphi)^{\dagger \rightarrow}}{\longrightarrow}\left\langle L_{2}^{X_{2}} \imath\right.$, where $\left((f, \varphi)^{\dagger \cdots}(\alpha)\right)\left(x_{2}\right)=$ $\varphi^{o p-1}\left(\bigvee_{f\left(x_{1}\right)=x_{2}} \alpha\left(x_{1}\right)\right)$.

(2) Let $\mathbf{L}$ be a variety, which extends $\operatorname{CSLat}(\bigvee)$, and let $\mathbf{S}$ be a subcategory of $\mathbf{L}^{o p}$ such that for every
S-morphism $L_{1} \stackrel{\varphi}{\rightarrow} L_{2}$, the map $\left|L_{1}\right| \stackrel{\varphi^{o p p}}{\longrightarrow}\left|L_{2}\right|$ is $\bigvee$ preserving. Then there exists the functor $\operatorname{Set} \times \mathbf{S} \stackrel{(-)^{\mapsto-}}{\longrightarrow}$ $\operatorname{CSLat}(\bigvee)$ defined by $\left(\left(X_{1}, L_{1}\right) \stackrel{(f, \varphi)}{\longrightarrow}\left(X_{2}, L_{2}\right)\right)^{\vdash-\rightarrow}=$ $\left\|L_{1}^{X_{1}}\right\| \stackrel{(f, \varphi)^{\vdash}}{\longrightarrow}\left\|L_{2}^{X_{2}}\right\|$, where $\left((f, \varphi)^{\vdash \cdots}(\alpha)\right)\left(x_{2}\right)=$ $\varphi^{o p \vdash}\left(\bigvee_{f\left(x_{1}\right)=x_{2}} \alpha\left(x_{1}\right)\right)$.

Proof. We rely on the fact that every $\Lambda$ - (resp., $\bigvee$-) semilattice is actually a complete lattice and, therefore, is a $\bigvee$ - (resp., $\Lambda$-) semilattice. The proof then follows the steps of those of Theorem 12, employing the fact that given CSLat $(\bigwedge)$ - (resp., $\operatorname{CSLat}(\bigvee)-)$ homomorphisms $L_{1} \stackrel{\varphi}{\rightarrow} L_{2}$ and $L_{2} \stackrel{\varphi^{\prime}}{\rightarrow} L_{3}$, it follows that $\left(\varphi^{\prime} \circ \varphi\right)^{-1}=\varphi^{-1} \circ \varphi^{\prime^{-1}}$ (resp., $\left.\left(\varphi^{\prime} \circ \varphi\right)^{\vdash}=\varphi^{\vdash} \circ \varphi^{\prime^{\prime}}\right)$.

The respective generalization of the backward powerset operator is even stronger (see, e.g., [21]), in the sense that the employed varieties do not need to be related to any kind of lattice-theoretic structure.

Theorem 14. Given a variety $\mathbf{A}$, every subcategory $\mathbf{S}$ of $\mathbf{A}^{o p}$ induces the functor Set $\times \mathbf{S} \stackrel{(-)^{\leftarrow}}{\longrightarrow} \mathbf{A}^{o p}$, which is defined by $\left(\left(X_{1}, A_{1}\right) \stackrel{(f, \varphi)}{\longrightarrow}\left(X_{2}, A_{2}\right)\right)^{\leftarrow}=A_{1}^{X_{1}} \stackrel{\left((f, \varphi)^{\leftarrow}\right)^{o p}}{\longrightarrow} A_{2}^{X_{2}}$, where $(f, \varphi)^{\leftarrow}(\alpha)=\varphi^{o p} \circ \alpha \circ f$.

Proof. To show that $A_{2}^{X_{2}} \stackrel{(f, \varphi)^{\leftarrow}}{\longrightarrow} A_{1}^{X_{1}}$ is an $\mathbf{A}$-homomorphism, notice that given $\lambda \in \Lambda_{\mathrm{A}}$ and $\left\langle\alpha_{i}\right\rangle_{n_{\lambda}} \in\left(A_{2}^{X_{2}}\right)^{n_{\lambda}}$, for every $x_{1} \in X_{1}$, it follows that

$$
\begin{aligned}
((f, \varphi) & \left.\leftarrow\left(\omega_{\lambda}^{A_{2}^{X_{2}}}\left(\left\langle\alpha_{i}\right\rangle_{n_{\lambda}}\right)\right)\right)\left(x_{1}\right) \\
& =\varphi^{o p} \circ\left(\omega_{\lambda}^{A_{2}^{X_{2}}}\left(\left\langle\alpha_{i}\right\rangle_{n_{\lambda}}\right)\right) \circ f\left(x_{1}\right) \\
& =\varphi^{o p} \circ\left(\omega_{\lambda}^{A_{2}}\left(\left\langle\alpha_{i} \circ f\left(x_{1}\right)\right\rangle_{n_{\lambda}}\right)\right) \\
& =\omega_{\lambda}^{A_{1}}\left(\left\langle\varphi^{o p} \circ \alpha_{i} \circ f\left(x_{1}\right)\right\rangle_{n_{\lambda}}\right) \\
& =\omega_{\lambda}^{A_{1}}\left(\left\langle\left((f, \varphi)^{\leftarrow}\left(\alpha_{i}\right)\right)\left(x_{1}\right)\right\rangle_{n_{\lambda}}\right) \\
& =\left(\omega_{\lambda}^{A_{1}^{X_{1}}}\left(\left\langle(f, \varphi)^{\leftarrow}\left(\alpha_{i}\right)\right\rangle_{n_{\lambda}}\right)\right)\left(x_{1}\right) .
\end{aligned}
$$

To show that the functor preserves composition, notice that given Set $\times \mathbf{S}$-morphisms $\left(X_{1}, A_{1}\right) \stackrel{(f, \varphi)}{\longrightarrow}\left(X_{2}, A_{2}\right)$ and $\left(X_{2}, A_{2}\right) \stackrel{\left(f^{\prime}, \varphi^{\prime}\right)}{\longrightarrow}\left(X_{3}, A_{3}\right)$, for every $\alpha \in A_{3}^{X_{3}},(f, \varphi)^{\leftarrow 。}$ $\left(f^{\prime}, \varphi^{\prime}\right)^{\leftarrow}(\alpha)=(f, \varphi)^{\leftarrow}\left(\varphi^{\prime o p} \circ \alpha \circ f^{\prime}\right)=\varphi^{o p} \circ \varphi^{\prime o p} \circ \alpha \circ f^{\prime} \circ f=$ $\left(\varphi^{\prime} \circ \varphi\right)^{\circ p} \circ \alpha \circ f^{\prime} \circ f=\left(f^{\prime} \circ f, \varphi^{\prime} \circ \varphi\right)^{\leftarrow}(\alpha)=\left(\left(f^{\prime}, \varphi^{\prime}\right) \circ\right.$ $(f, \varphi))^{\leftarrow}(\alpha)$.

Similar to the case of variety-based forward powerset operators, we have the following modifications of the functor introduced in Theorem 14.

Theorem 15. Suppose that either $\left(\Xi_{1}, \Xi_{2}, \dagger\right)=(\bigvee, \Lambda, \vdash)$ or $\left(\Xi_{1}, \Xi_{2}, \dagger\right)=(\bigwedge, \bigvee, \dashv)$. Given a variety $\mathbf{L}$, which extends 
$\operatorname{CSLat}\left(\Xi_{1}\right)$, every subcategory $\mathbf{S}$ of $\mathbf{L}$ provides the functor $\operatorname{Set} \times$ $\mathbf{S} \stackrel{(-)^{\dagger+}}{\longrightarrow}\left(\operatorname{CSLat}\left(\Xi_{2}\right)\right)^{o p}$, which is defined by $\left(\left(X_{1}, L_{1}\right) \stackrel{(f, \varphi)}{\longrightarrow}\right.$ $\left.\left(X_{2}, L_{2}\right)\right)^{\dagger+\cdots}=2 L_{1}^{X_{1}} 2 \stackrel{\left((f, \varphi)^{\dagger \cdots}\right)^{o p}}{\longrightarrow} 2 L_{2}^{X_{2}}$, where $(f, \varphi)^{\dagger \cdots \cdots}(\alpha)=$ $\varphi^{\dagger} \circ \alpha \circ f$.

Proof. The proof is a straightforward modification of that of Theorem 14.

In the paper, we will use the following dualized versions of the functors of Theorems 12 and 13.

\section{Theorem 16.}

(1) Given a variety $\mathbf{L}$, which extends $\operatorname{CSLat}(\bigvee)$, every subcategory $\mathbf{S}$ of $\mathbf{L}^{\text {op }}$ gives rise to the functor Set $^{o p} \times$ $\mathbf{S} \stackrel{(-)^{\rightarrow o}}{\longrightarrow}(\operatorname{CSLat}(\bigvee))^{o p}$ defined by $\left(\left(X_{1}, L_{1}\right) \stackrel{(f, \varphi)}{\longrightarrow}\right.$ $\left.\left(X_{2}, L_{2}\right)\right)^{\rightarrow o}=\left\|L_{1}^{X_{1}}\right\| \stackrel{\left((f, \varphi)^{\rightarrow o}\right)^{o p}}{\longrightarrow}\left\|L_{2}^{X_{2}}\right\|$, where $\left((f, \varphi)^{\rightarrow o}(\alpha)\right)\left(x_{1}\right)=\varphi^{o p}\left(\bigvee_{f^{o p}\left(x_{2}\right)=x_{1}} \alpha\left(x_{2}\right)\right)$.

(2) Given a variety $\mathbf{L}$, which extends $\operatorname{CSLat}(\Lambda)$, every subcategory $\mathbf{S}$ of $\mathbf{L}$ gives rise to the functor $\mathbf{S e t}^{o p} \times$ $\mathbf{S} \stackrel{(-)^{\dagger \rightarrow o}}{\longrightarrow}(\operatorname{CSLat}(\bigvee))^{o p}$ defined by $\left(\left(X_{1}, L_{1}\right) \stackrel{(f, \varphi)}{\longrightarrow}\right.$ $\left.\left(X_{2}, L_{2}\right)\right)^{\dashv \rightarrow \infty}=\imath L_{1}^{X_{1}} 2 \stackrel{\left((f, \varphi)^{\dagger \rightarrow 0}\right)^{o p}}{\longrightarrow} \imath L_{2}^{X_{2}}$, where $\left((f, \varphi)^{\dagger \rightarrow \infty}(\alpha)\right)\left(x_{1}\right)=\varphi^{-1}\left(\bigvee_{f^{o p}\left(x_{2}\right)=x_{1}} \alpha\left(x_{2}\right)\right)$.

(3) Let $\mathbf{L}$ be a variety, which extends $\operatorname{CSLat}(\bigvee)$, and let $\mathbf{S}$ be a subcategory of $\mathbf{L}$ such that for every $\mathbf{S}$ morphism $L_{1} \stackrel{\varphi}{\rightarrow} L_{2}$, the map $\left|L_{2}\right| \stackrel{\varphi^{\vdash}}{\rightarrow}\left|L_{1}\right|$ is $\bigvee$-preserving. Then there exists the functor Set $^{o p} \times$ $\mathbf{S} \stackrel{(-)^{\vdash \cdots o}}{\longrightarrow}(\operatorname{CSLat}(\bigvee))^{o p}$ defined by $\left(\left(X_{1}, L_{1}\right) \stackrel{(f, \varphi)}{\longrightarrow}\right.$ $\left.\left(X_{2}, L_{2}\right)\right)^{\vdash \rightarrow o}=\left\|L_{1}^{X_{1}}\right\| \stackrel{\left((f, \varphi)^{\vdash \rightarrow o}\right)^{o p}}{\longrightarrow}\left\|L_{2}^{X_{2}}\right\|$, where $\left((f, \varphi)^{\vdash \cdots o}(\alpha)\right)\left(x_{1}\right)=\varphi^{\vdash}\left(\bigvee_{f^{o p}\left(x_{2}\right)=x_{1}} \alpha\left(x_{2}\right)\right)$.

Additionally, the functors of Theorems 14 and 15 can be dualized as follows.

\section{Theorem 17.}

(1) Given a variety $\mathbf{A}$, every subcategory $\mathbf{S}$ of $\mathbf{A}$ induces the functor $\mathbf{S e t}^{o p} \times \mathbf{S} \stackrel{(-)^{\leftarrow o}}{\longrightarrow} \mathbf{A}$, which is defined by $\left(\left(X_{1}, A_{1}\right) \stackrel{(f, \varphi)}{\longrightarrow}\left(X_{2}, A_{2}\right)\right)^{\leftarrow o}=A_{1}^{X_{1}} \stackrel{(f, \varphi)^{\leftarrow o}}{\longrightarrow} A_{2}^{X_{2}}$, where $(f, \varphi)^{\leftarrow o}(\alpha)=\varphi \circ \alpha \circ f^{o p}$.

(2) Suppose that either $\left(\Xi_{1}, \Xi_{2}, \dagger\right)=(\bigvee, \bigwedge, \vdash)$ or $\left(\Xi_{1}, \Xi_{2}, \dagger\right)=(\bigwedge, \bigvee,-1)$. Given a variety $\mathbf{L}$, which extends $\operatorname{CSLat}\left(\Xi_{1}\right)$, every subcategory $\mathbf{S}$ of $\mathbf{L}^{\text {op }}$ provides the functor $\operatorname{Set}^{o p} \times \mathbf{S} \stackrel{(-)^{\dagger+\infty}}{\longrightarrow} \operatorname{CSLat}\left(\Xi_{2}\right)$, which is defined by $\left(\left(X_{1}, L_{1}\right) \stackrel{(f, \varphi)}{\longrightarrow}\left(X_{2}, L_{2}\right)\right)^{\dagger \cdots o}=$

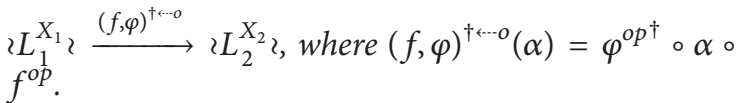

The reader should notice that the functors of Theorem 17 are not standard in the fuzzy community.
2.3. Galois Connections. The paper relies heavily on the welldeveloped machinery of Galois connections. For convenience of the reader, we recall from $[32,37,50]$ their basic definitions.

Definition 18. An order-preserving (resp., -reversing) Galois connection is a tuple $\left(\left(X_{1}, \leqslant\right), f, g,\left(X_{2}, \leqslant\right)\right)$, where $\left(X_{1}, \leqslant\right)$, $\left(X_{2}, \leqslant\right)$ are posets and $X_{1} \underset{g}{\stackrel{f}{\rightleftarrows}} X_{2}$ are maps such that for every $x_{1} \in X_{1}$ and every $x_{2} \in X_{2}, x_{1} \leqslant g\left(x_{2}\right)$ iff $f\left(x_{1}\right) \leqslant x_{2}$ (resp., $x_{1} \leqslant g\left(x_{2}\right)$ iff $\left.x_{2} \leqslant f\left(x_{1}\right)\right)$.

As a consequence, one easily gets the following wellknown features of Galois connections.

Theorem 19. Every order-preserving (resp., order-reversing) Galois connection $\left(\left(X_{1}, \leqslant\right), f, g,\left(X_{2}, \leqslant\right)\right)$ has the following properties.

(1) $1_{X_{1}} \leqslant g \circ f$ and $f \circ g \leqslant 1_{X_{2}}$ (resp., $1_{X_{1}} \leqslant g \circ f$ and $\left.1_{X_{2}} \leqslant f \circ g\right)$.

(2) Both $f$ and $g$ are order-preserving (resp., orderreversing).

(3) $f(\bigvee S)=\bigvee_{s \in S} f(s)$ and $g(\bigwedge T)=\bigwedge_{t \in T} g(t)$ (resp., $f(\bigvee S)=\bigwedge_{s \in S} f(s)$ and $\left.g(\bigvee T)=\bigwedge_{t \in T} g(t)\right)$ provided that the joins and meets in question exist in the domains.

(4) $g \circ f \circ g=g$ and $f \circ g \circ f=f$.

Notice that the second item of Theorem 19 is responsible for the term "order-preserving" (resp., "order-reversing") in Definition 18. Moreover, in view of the results of Theorem 19, there exists the following equivalent definition of Galois connections.

Definition 20. An order-preserving (resp., orderreversing) Galois connection is provided by a tuple $\left(\left(X_{1}, \leqslant\right), f, g,\left(X_{2}, \leqslant\right)\right)$, where $\left(X_{1}, \leqslant\right),\left(X_{2}, \leqslant\right)$ are posets and $\left(X_{1}, \leqslant\right) \underset{g}{\stackrel{f}{\rightleftarrows}}\left(X_{2}, \leqslant\right)$ are order-preserving (resp., orderreversing) maps such that $1_{X_{1}} \leqslant g \circ f$ and $f \circ g \leqslant 1_{X_{2}}$ (resp., $1_{X_{1}} \leqslant g \circ f$ and $1_{X_{2}} \leqslant f \circ g$ ).

To give the flavor of these notions, we show some examples of order-preserving Galois connections already encountered in the paper. An important example of orderreversing Galois connections will be encountered later on in the paper.

\section{Example 21.}

(1) Every $\bigvee$-semilattice (resp., $\bigwedge$-semilattice) homomorphism $A_{1} \stackrel{\varphi}{\rightarrow} A_{2}$ provides the order-preserving Galois connection $\left(A_{1}, \varphi, \varphi^{\vdash}, A_{2}\right)$ (resp., $\left(A_{2}, \varphi^{\vdash}\right.$, $\left.\left.\varphi, A_{1}\right)\right)$.

(2) Every element $q$ of a quantale $Q$ provides the orderpreserving Galois connections $\left(Q, \cdot \otimes q, q \rightarrow l_{l}, Q\right)$ and $\left(Q, q \otimes \cdot, q \rightarrow_{r} \cdot, Q\right)$. 
(3) Every map $X_{1} \stackrel{f}{\rightarrow} X_{2}$ provides the order-preserving Galois connection $\left(\mathscr{P}\left(X_{1}\right), f^{\rightarrow}, f^{\leftarrow}, \mathscr{P}\left(X_{2}\right)\right)$.

(4) Given a variety L extending $\operatorname{CSLat}(\bigvee)$, every Set $\times$ L-morphism $\left(X_{1}, L_{1}\right) \stackrel{(f, \varphi)}{\longrightarrow}\left(X_{2}, L_{2}\right)$ provides the order-preserving Galois connection $\left(L_{1}^{X_{1}},(f, \varphi) \rightarrow,(f, \varphi)^{\vdash \cdots \cdots}, L_{2}^{X_{2}}\right)$.

To conclude, we would like to notice that this paper studies the properties of the classical Galois connections on lattice-valued powersets. In contrast [38, 40], employ the notion of fuzzy Galois connection, which is particularly designed to fit the lattice-valued powerset framework. Such a modification is needed to preserve the main results valid on crisp powersets. The preservation in question comes, however, at the expense of a more complicated definition of fuzzy Galois connections themselves, which will be off our topic of study.

\section{Variety-Based Topological Systems and Their Modifications}

In [1], we introduced the concept of variety-based topological systems as a generalization of Vickers' topological systems [2] mentioned in the Introduction section. Moreover, in [22], we presented a modified category of variety-based topological systems, to accommodate Vickers' system localification procedure. For convenience of the reader, we recall both definitions, restating them, however, in a slightly more general way, to suit the framework of the current paper.

Definition 22. Given a variety $\mathbf{A}$, a subcategory $\mathbf{S}$ of $\mathbf{A}^{o p}$, and a reduct $\mathbf{B}$ of $\mathbf{A}, \mathbf{S}_{\mathbf{B}}$-TopSys is the category, concrete over the product category Set $\times \mathbf{B}^{o p} \times \mathbf{S}$, which comprises the following data.

Objects. Tuples $D=($ pt $D, \Omega D, \Sigma D, \vDash)\left(\mathbf{S}_{\mathbf{B}}\right.$-topological systems or $\mathbf{S}_{\mathbf{B}}$-systems), where ( $\left.p t D, \Omega D, \Sigma D\right)$ is a Set $\times \mathbf{B}^{o p} \times \mathbf{S}$ object, whereas pt $D \times \Omega D \stackrel{\vDash}{\rightarrow} \Sigma D$ is a map ( $\Sigma D$-satisfaction relation on $(\operatorname{pt} D, \Omega D))$ such that $\Omega D \stackrel{\vDash(x,-)}{\longrightarrow}\|\Sigma D\|$ is a $\mathbf{B}$ homomorphism for every $x \in$ pt $D$.

Morphisms. $D_{1} \stackrel{f=(\text { pt } f, \Omega f, \Sigma f)}{\longrightarrow} D_{2}$ are all those Set $\times \mathbf{B}^{o p} \times \mathbf{S}$ morphisms (pt $\left.D_{1}, \Omega D_{1}, \Sigma D_{1}\right) \stackrel{f}{\rightarrow}\left(\right.$ pt $D_{2}, \Omega D_{2}, \Sigma D_{2}$ ), which satisfy the property $\vDash_{1}\left(x,(\Omega f)^{o p}(b)\right)=\left(\sum f\right)^{o p}{ }_{\circ}^{\circ} \vDash_{2}($ pt $f(x), b)$ for every $x \in \operatorname{pt} D_{1}$ and every $b \in \Omega D_{2}$ ( $\mathbf{S}_{\mathrm{B}}$-continuity).

The following notational remark applies to all the categories of systems introduced in this paper.

Remark 23. The case $\mathbf{S}=\mathbf{S}_{A}$ is called (variety-based) fixedbasis approach, whereas all other cases are subsumed under (variety-based) variable-basis approach. Moreover, if $\mathbf{A}=$ $\mathbf{B}$, then the notation $\mathbf{S}_{\mathbf{B}}$-TopSys is truncated to $\mathbf{S}$-TopSys, whereas if $\mathbf{S}=\mathbf{S}_{A}$, then the notation is shortened to $A_{\mathrm{B}}$-TopSys. In the latter case, the objects (resp., morphisms) are truncated to $D=(\operatorname{pt} D, \Omega D, \vDash)($ resp., $f=(\operatorname{pt} f, \Omega f))$.
The next example gives the reader the intuition for the new category, showing that the latter incorporates many of the already existing concepts in the literature.

Example 24.

(1) If $\mathbf{A}=\mathbf{C B A l g}$, then the category $2 \mathrm{Frm}$-TopSys, where $2=\{\perp, \mathrm{T}\}$ is the two-element complete Boolean algebra, is isomorphic to the category TopSys of topological systems of Vickers [2].

(2) If $\mathbf{A}=\mathbf{B}=$ Frm, then the category Loc-TopSys is the category of lattice-valued topological systems of Denniston et al. $[8,9]$.

(3) If $\mathbf{A}=\mathbf{C Q u a n t}$, then the category $A_{\text {Set }}$-TopSys is the category $A$-IntSys of lattice-valued interchange systems of Denniston et al. [11, 29, 30], which is also the category A-TransSys of lattice-valued transformer systems of Denniston et al. [15] (notice that both categories are fixed-basis).

(4) If $\mathbf{A}=\mathbf{B}=$ Set, then the category $A$-TopSys is the category $\mathbf{C h u}_{A}$ of $C h u$ spaces over the set $A$ of Pratt [13]. In particular, the objects of $\mathbf{C h u}_{2}$ are called formal contexts in Formal Concept Analysis of Ganter and Wille [31], whereas the objects of the category $\mathbf{C h u}_{A}$ itself are called many-valued formal contexts [31, Section 1.3]. Additionally, the respective context morphisms proposed by Ganter and Wille [31, Chapter 7] are different from the morphisms of Chu spaces, which will be dealt with in the subsequent sections of the paper.

Moreover, the following definition (extended and adapted for the current setting from [15]) illustrates the concept of a topological system morphism.

Definition 25. Letting A = CBAlg, GalCon (notice that "GalCon" stands for "Galois connections") is the full subcategory of the category $2_{\text {Set }}$-TopSys, whose objects are those 2 set-topological systems $D$, which satisfy the following two conditions:

(1) pt $D=\Omega D=X$;

$(2) \vDash$ is a partial order on $X$.

The reader can easily see (or verify) that the objects of the category GalCon are posets, and its morphisms are precisely the order-preserving Galois connections, which is reflected in its name. This category is essentially different from the category GAL of Galois connections of [32], in the sense that while the latter category uses Galois connections as its objects, the category GalCon uses them as morphisms. It will be the topic of our future research, to consider the properties of the category GalCon (at least) up to the extent of the respective ones of the category GAL. Notice, however, that the topic of the category GAL will find its proper place in the subsequent developments of the paper.

The reader should also be aware of the important fact that the case of order-reversing Galois connections 
$\left(X_{1}, \leqslant\right) \underset{g}{\stackrel{f}{\rightleftarrows}}\left(X_{2}, \leqslant\right)$ (up to the knowledge of the author) does not allow the previously mentioned system interpretation. Indeed, the already mentioned adjunction condition now reads as $x_{1} \leqslant g\left(x_{2}\right)$ iff $x_{2} \leqslant f\left(x_{1}\right)$ for every $x_{1} \in X_{1}$ and every $x_{2} \in X_{2}$. With the help of the dual partial order $\leqslant^{d}$ on $X_{2}$, we do get an order-preserving Galois connection $\left(X_{1}, \leqslant\right) \underset{g}{\stackrel{f}{\rightleftarrows}}\left(X_{2}, \leqslant^{d}\right)$. It is unclear though how to incorporate in the system setting another order-reversing Galois connection $\left(X_{2}, \leqslant\right) \underset{g^{\prime}}{\stackrel{f^{\prime}}{\rightleftarrows}}\left(X_{3}, \leqslant\right)$ (or $\left.\left(X_{2}, \leqslant\right) \underset{g^{\prime}}{\stackrel{f^{\prime}}{\rightleftarrows}}\left(X_{3}, \leqslant^{d}\right)\right)$, that is, how to define the morphism composition law of the possible category (notice that $\left(X_{2}, \leqslant\right)$ and $\left(X_{2}, \leqslant^{d}\right)$ are different as posets; i.e., the codomain of the first connection is not the domain of the second one).

In the next step, we introduce the modified category of topological systems inspired by [22].

Definition 26. Given a variety $\mathbf{A}$, a subcategory $\mathbf{S}$ of $\mathbf{A}$,

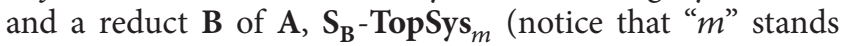
for "modified") is the category, concrete over the product category Set $\times \mathbf{B}^{o p} \times \mathbf{S}$, which comprises the following data.

Objects. Tuples $D=(\operatorname{pt} D, \Omega D, \Sigma D, \vDash)$, where (pt $D, \Omega D, \Sigma D)$ is a Set $\times \mathbf{B}^{o p} \times \mathbf{S}$-object and pt $D \times \Omega D \stackrel{\vDash}{\rightarrow} \Sigma D$ is a map such that $\Omega D \stackrel{\vDash(x,-)}{\longrightarrow}\|\Sigma D\|$ is a $\mathbf{B}$-homomorphism for every $x \in \operatorname{pt} D$.

Morphisms. $D_{1} \stackrel{f=(\operatorname{pt} f, \Omega f, \Sigma f)}{\longrightarrow} D_{2}$ are Set $\times \mathbf{B}^{o p} \times \mathbf{S}$-morphisms $\left(\right.$ pt $\left.D_{1}, \Omega D_{1}, \Sigma D_{1}\right) \stackrel{f}{\rightarrow}\left(\operatorname{pt} D_{2}, \Omega D_{2}, \Sigma D_{2}\right)$ such that $\Sigma f \circ$ $F_{1}\left(x,(\Omega f)^{o p}(b)\right)=\vDash_{2}($ pt $f(x), b)$ for every $x \in$ pt $D_{1}$ and every $b \in \Omega D_{2}$.

Since both a category and its dual have the same objects, the only difference between the categories of systems introduced in Definitions 22 and 26 is in the direction of the $\sum$ component of their respective morphisms. In particular, with the notational conventions of Remark 23, we get that all the items of Example 24, except the second one, can be restated in terms of the category $\mathbf{S}_{\mathbf{B}}$-TopSys $\mathbf{s}_{m}$. Moreover, it is easy to provide the conditions, under which the system settings of Definitions 22 and 26 coincide.

Definition 27. Given a subcategory $\mathbf{S}$ of $\mathbf{A}^{o p}, \mathbf{S}_{\mathbf{B}}$-TopSys ${ }^{i}$ (resp., $\mathbf{S}_{\mathbf{B}}^{o p}$-TopSys ${ }_{m}^{i}$ ) is the nonfull subcategory of the category $\mathbf{S}_{\mathbf{B}}$-TopSys (resp., $\mathbf{S}_{\mathbf{B}}^{o p}$-TopSys $\mathbf{s}_{m}$ ), which has the same objects and whose morphisms $D_{1} \stackrel{f}{\rightarrow} D_{2}$ are such that $\Sigma D_{1} \stackrel{\Sigma f}{\longrightarrow} \Sigma D_{2}$ (resp., $\Sigma D_{1} \stackrel{(\Sigma f)^{o p}}{\longrightarrow} \Sigma D_{2}$ ) is an $\mathbf{S}$ - (resp., $\mathbf{S}^{o p}$ ) isomorphism.

Theorem 28. There exists the isomorphism $\mathbf{S}_{\mathbf{B}}$-TopSys $\stackrel{i}{\rightarrow}$ $\mathbf{S}_{\mathbf{B}}^{\text {op }}$-TopSys $s_{m}^{i}$, which is defined by the formula $F\left(D_{1} \stackrel{f}{\rightarrow} D_{2}\right)=$ $D_{1} \stackrel{\left(p t f, \Omega f,\left((\Sigma f)^{o p}\right)^{-1}\right)}{\longrightarrow} D_{2}$.
Proof. Straightforward computations.

As a consequence, we obtain that there is no difference between the (fixed-basis) categories $A_{\mathbf{B}}$-TopSys and $A_{\mathbf{B}}$-TopSys $s_{m}$. There exists, however, another obvious modification of the category of topological systems, which has never appeared in the literature before.

Definition 29. Given a variety $\mathbf{A}$, a subcategory $\mathbf{S}$ of $\mathbf{A}^{o p}$, and a reduct $\mathbf{B}$ of $\mathbf{A}, \mathbf{S}_{\mathbf{B}}$-TopSys T $_{a}$ (notice that " $a$ " stands for "alternative") is the category, concrete over the product category Set $\times \mathbf{B} \times \mathbf{S}$, which comprises the following data.

Objects. Tuples $D=(\operatorname{pt} D, \Omega D, \Sigma D, \vDash)$, where (pt $D, \Omega D, \Sigma D)$ is a Set $\times \mathbf{B} \times \mathbf{S}$-object, whereas pt $D \times \Omega D \stackrel{\vDash}{\rightarrow} \Sigma D$ is a map such that $\Omega D \stackrel{\vDash(x,-)}{\longrightarrow}\|\Sigma D\|$ is a $\mathbf{B}$-homomorphism for every $x \in$ pt $D$.

Morphisms. $D_{1} \stackrel{f=(\mathrm{pt} f, \Omega f, \Sigma f)}{\longrightarrow} D_{2}$ are Set $\times \mathbf{B} \times$ S-morphisms $\left(\operatorname{pt} D_{1}, \Omega D_{1}, \Sigma D_{1}\right) \stackrel{f}{\rightarrow} \quad\left(\operatorname{pt~} D_{2}, \Omega D_{2}, \Sigma D_{2}\right)$ such that $\vDash_{1}(x, b)=(\Sigma f)^{o p} \circ \vDash_{2}($ pt $f(x), \Omega f(b))$ for every $x \in \operatorname{pt} D_{1}$ and every $b \in \Omega D_{1}$.

It is important to emphasize that leaving (essentially) the same objects, we change the definition of morphisms dramatically. In particular, we cannot obtain a single item of Example 24 in the new framework.

Similar to the previously mentioned classical system setting, we can introduce a modified category of systems.

Definition 30. Given a variety $\mathbf{A}$, a subcategory $\mathbf{S}$ of $\mathbf{A}$, and a reduct $\mathbf{B}$ of $\mathbf{A}, \mathbf{S}_{\mathbf{B}}$-TopSys $\mathbf{s}_{a m}$ is the category, concrete over the product category Set $\times \mathbf{B} \times \mathbf{S}$, which comprises the following data.

Objects. Tuples $D=(\operatorname{pt} D, \Omega D, \Sigma D, \vDash)$, where (pt $D, \Omega D, \Sigma D)$ is a Set $\times \mathbf{B} \times \mathbf{S}$-object and pt $D \times \Omega D \stackrel{\vDash}{\rightarrow} \Sigma D$ is a map such that $\Omega D \stackrel{\vDash(x,-)}{\longrightarrow}\|\Sigma D\|$ is a B-homomorphism for every $x \in \operatorname{pt} D$.

Morphisms. $D_{1} \stackrel{f=(\operatorname{pt~} f, \Omega f, \Sigma f)}{\longrightarrow} D_{2}$ are Set $\times \mathbf{B} \times \mathbf{S}$-morphisms $\left(\operatorname{pt} D_{1}, \Omega D_{1}, \Sigma D_{1}\right) \stackrel{f}{\rightarrow}\left(\operatorname{pt} D_{2}, \Omega D_{2}, \Sigma D_{2}\right)$ such that $\Sigma f$ 。 $\vDash_{1}(x, b)=\vDash_{2}$ (pt $\left.f(x), \Omega f(b)\right)$ for every $x \in$ pt $D_{1}$ and every $b \in \Omega D_{1}$.

The explicit restatement of Theorem 28 for the setting of the categories $\mathbf{S}_{\mathbf{B}}$-TopSys $\mathbf{s}_{a}, \mathbf{S}_{\mathbf{B}}$-TopSys $\mathbf{s}_{a m}$ is straightforward and is, therefore, left to the reader.

\section{Lattice-Valued Formal Concept Analysis}

For the required preliminaries in hand, in this section, we introduce different approaches to fuzzification of basic tools of Formal Concept Analysis (FCA) of Ganter and Wille [31]. In particular, we present three possible category-theoretic machineries, the first two of which stem from the approach of Denniston et al. [29], whereas the last one comes from Ganter 
and Wille themselves. For the sake of brevity, we omitted every motivational reason for the need of fuzziness in FCA, which is clearly stated in, for example, [33], and also could be found at the beginning of Section 5 of [29]. Our main interest is not the idea of FCA itself, but rather a better categorytheoretic framework for its successful development.

4.1. Lattice-Valued Formal Contexts as Chu Spaces. In the last item of Example 24, we represented Chu spaces over sets of Pratt [13] as particular instances of variety-based topological systems. Moreover, we also mentioned that one of the main building blocks of FCA, that is, (many-valued) formal contexts, is, in fact, nothing else than Chu spaces (already noticed in the literature $[55,56]$ ). With these ideas in mind, we introduce the following definition.

Definition 31. Let $\mathbf{L}$ be an extension of the variety Quant of quantales and let $\mathbf{B}=$ Set.

(1) S-FC $C$ (notice that " $C$ " stands for "Chu") is a new notation for the category $\mathbf{S}_{\text {Set }}$-TopSys, whose objects ((lattice-valued) formal contexts) are now denoted $\mathscr{K}=(G, M, L, I)$ (in the notation of Definition 22, $\mathscr{K}=D$, which yields $G=$ pt $D, M=\Omega D$, $L=\Sigma D$ and $I=\vDash$ ), where $G$ (from German "Gegenstände") is the set of context objects, $M$ (from German "Merkmale") is the set of context attributes, and $I$ (from German "Inzidenzrelation") is the context incidence relation. The respective morphisms ((latticevalued) formal context morphisms) $\mathscr{K}_{1} \stackrel{f}{\rightarrow} \mathscr{K}_{2}$ are now triples $\left(G_{1}, M_{1}, L_{1}\right) \stackrel{f=(\alpha, \beta, \varphi)}{\longrightarrow}\left(G_{2}, M_{2}, L_{2}\right)$ (in the notation of Definition 22, $\alpha=$ pt $f, \beta=\Omega f$ and $\varphi=\Sigma f$, and then, for every $g \in G_{1}$ and every $\left.m \in M_{2}, I_{1}\left(g, \beta^{o p}(m)\right)=\varphi^{o p} \circ I_{2}(\alpha(g), m)\right)$. The case of $\mathbf{L}=\mathbf{C B A l g}$ and $\mathbf{S}=\mathbf{S}_{2}$ is called the crisp case.

(2) $\mathbf{S}-\mathbf{F C}_{m}^{C}$ is a new notation for the category $\mathbf{S}_{\mathbf{B}}$-TopSys $\mathbf{S}_{m}$.

The notational and naming remarks for objects and morphisms of Definition 31 will apply to all the categories of (lattice-valued) formal contexts introduced in this paper. Moreover, the reader can easily apply Theorem 28, to single out the isomorphic subcategories of the categories $\mathbf{S}-\mathbf{F C}^{C}$ and $\mathbf{S}-\mathbf{F C}_{m}^{C}$.

At the moment, there is no agreement in the fuzzy community, whether to build formal contexts over $\mathbf{L}$ or $\mathbf{L}^{o p}$, and, therefore, we just add the lower index " $(-)_{m}$ " in the former case. More precisely, this paper is (up to the knowledge of the author) the first attempt to develop a variable-basis approach (a la [4]) to lattice-valued FCA, the motivational article of Denniston et al. [29] being fixed-basis.

4.2. Lattice-Valued Formal Contexts in the Sense of Ganter and Wille. In the previous subsection, we presented a latticevalued approach to FCA based in Chu spaces. An experienced reader, however, may be well aware of many-valued contexts of Ganter and Wille themselves [31, Section 13], the theory of which is already established in the literature. In the following, we provide its category-theoretic analogue, bringing together both many-valued contexts and their morphisms.

Definition 32. Let $\mathbf{L}$ be an extension of Quant and let $\mathbf{B}=$ Set.

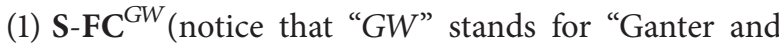
Wille") is a new notation for the category $\mathbf{S}_{\mathbf{B}}-$ TopSys $_{a}$.

(2) $\mathbf{S}-\mathbf{F C}_{m}^{G W}$ is a new notation for the category $\mathrm{S}_{\mathrm{B}}$-TopSys $\mathbf{S}_{a m}$.

It should be emphasized immediately that neither Ganter nor Wille (up to the knowledge of the author) has considered a categorical approach to (many-valued) formal contexts, that is, has united both formal contexts and their morphisms in one entity. Additionally, both (many-valued) formal contexts and their morphisms are treated in an algebraic way in [31] (from where our notation for context morphisms, which is different from the system setting, is partly borrowed). There does exist some research on certain categories of contexts (e.g., [57-61]), which, however, does not study the categorical foundation for FCA, but rather concentrates on a fixed categorical framework and its related results. It is the main purpose of this paper, to investigate such possible categorytheoretic foundations and their relationships to each other (leaving their related results to the further study on the topic). In particular, we consider a category of contexts, whose morphisms are strikingly different from the standard framework of topological systems.

\subsection{Lattice-Valued Formal Contexts in the Sense of Denniston} et al. In [29], Denniston et al. have introduced another approach to lattice-valued FCA, which was based on the category GAL of order-preserving Galois connections of [32]. In the following, we extend their approach in two respects. Firstly, we employ the variety Quant of quantales instead of CQuant of commutative ones of [29] (called there complete commutative residuated lattices). Secondly, we provide a variable-basis approach (à la [4]) to the topic, instead of the fixed-based one of [29].

To begin with, we introduce some new notions and notations (induced by the respective ones of [29]), which will be used in the subsequent developments of the paper.

Definition 33. Every lattice-valued formal context $\mathscr{K}$ has the following (lattice-valued) Birkhoff operators:

$$
\begin{aligned}
& \text { (1) } L^{G} \stackrel{H}{\longrightarrow} L^{M} \text {, which is given by }(H(s))(m)= \\
& \bigwedge_{g \in G}\left(s(g) \rightarrow{ }_{l} I(g, m)\right) \text {, } \\
& \text { (2) } L^{M} \stackrel{K}{\longrightarrow} L^{G} \text {, which is given by }(K(t))(g)= \\
& \bigwedge_{m \in M}\left(t(m) \rightarrow{ }_{r} I(g, m)\right) \text {. }
\end{aligned}
$$

Given a lattice-valued context $\mathscr{K}$, the notation $s$ for the elements of $L^{G}$ (resp., $t$ for the elements of $L^{M}$ ), that is, latticevalued sets of context objects (resp., attributes), will be used throughout the paper.

To give the reader more intuition, we provide an example of the just introduced notions. 
Example 34. Every crisp context $\mathscr{K}$ (recall our crispness remark from Definition 31(1)) provides the maps

(1) $\mathscr{P}(G) \stackrel{H}{\rightarrow} \mathscr{P}(M)$, which is given by $H(S)=\{m \in M \mid$ $s I m$ for every $s \in S\}$,

(2) $\mathscr{P}(M) \stackrel{K}{\longrightarrow} \mathscr{P}(G)$, which is given by $K(T)=\{g \in G \mid$ $g I t$ for every $t \in T\}$,

which are the classical Birkhoff operators generated by a binary relation.

An important property of the maps of Definition 33 is contained in the following result.

Theorem 35. For every lattice-valued context $\mathscr{K}$, $\left(L^{G}, H, K, L^{M}\right)$ is an order-reversing Galois connection.

Proof. For convenience of the reader, we provide the proof, which is different from that of [29].

To show that the map $H$ is order-reversing, notice that given $s_{1}, s_{2} \in L^{G}$ such that $s_{1} \leqslant s_{2}$, and some $m \in M$, for every $g \in G$, it follows that $s_{2}(g) \rightarrow{ }_{l} I(g, m) \leqslant s_{1}(g) \rightarrow{ }_{l} I(g, m)$ (Theorem 6(2)) and, therefore, $\left(H\left(s_{2}\right)\right)(m)$

$\bigwedge_{g \in G}\left(s_{2}(g) \rightarrow{ }_{l} I(g, m)\right) \leqslant \bigwedge_{g \in G}\left(s_{1}(g) \rightarrow{ }_{l} I(g, m)\right)=$ $\left(H\left(s_{1}\right)\right)(m)$. The case of $K$ is similar.

To show that $1_{L^{G}} \leqslant K \circ H$, notice that given $s \in L^{G}$ and $g \in G$, it follows that $(K \circ H(s))(g)=$ $\bigwedge_{m \in M}\left((H(s))(m) \rightarrow{ }_{r} I(g, m)\right)=\bigwedge_{m \in M}\left(\left(\bigwedge_{g^{\prime} \in G}\left(s\left(g^{\prime}\right) \rightarrow_{l}\right.\right.\right.$ $\left.\left.\left.I\left(g^{\prime}, m\right)\right)\right) \rightarrow{ }_{r} I(g, m)\right) \geqslant \bigwedge_{m \in M}\left(\left(s(g) \rightarrow{ }_{l} I(g, m)\right) \rightarrow{ }_{r}\right.$ $I(g, m)) \stackrel{(\dagger)}{\geqslant} \bigwedge_{m \in M} s(g) \geqslant s(g)$, where $(\dagger)$ relies on the first claim of Theorem 6(3). The proof of $1_{L^{M}} \leqslant H \circ K$ uses the dual machinery and the second claim of Theorem 6(3).

We emphasize immediately that Theorem 35 is an analogue of the already obtained results of [38, Lemma 3] (for $L$ a complete residuated lattice) and of [40, Proposition 3.9] (for $L$ a unital quantale) in the framework of fuzzy Galois connections, which is different (more demanding) from the setting of Galois connections of the current paper (see additionally the remark after Theorem 42). There also exists an extension of Theorem 35 to a more general context of (commutative, right-distributive, complete) extended-order algebras $L$ (whose multiplication in some cases need not be associative) [62, Propositions 3.9, 5.5] and, additionally, to even more general case, which involves relations instead of maps (the so-called L-Galois triangles) [63].

Everything is now in hand, to define another latticevalued extension of formal contexts of FCA.

Definition 36. Given a variety $\mathbf{L}$, which extends Quant, and a subcategory $\mathbf{S}$ of $\mathbf{L}, \mathbf{S}-\mathbf{F} C^{D M R}$ (notice that "DMR" stands for "Denniston, Melton and Rodabaugh") is the category, concrete over the product category Set $\times$ Set $^{o p}$, which comprises the following data.

Objects. Lattice-valued formal contexts $\mathscr{K}$ (in the sense of Definition 31) such that $L$ is an object of $\mathbf{S}$.
Morphisms. $\mathscr{K}_{1} \stackrel{f=(\alpha, \beta)}{\longrightarrow} \mathscr{K}_{2}$ are Set $\times$ Set $^{o p}$-morphisms $\left(L_{1}^{G_{1}}, L_{1}^{M_{1}}\right) \stackrel{(\alpha, \beta)}{\longrightarrow}\left(L_{2}^{G_{2}}, L_{2}^{M_{2}}\right)$, which make the diagrams

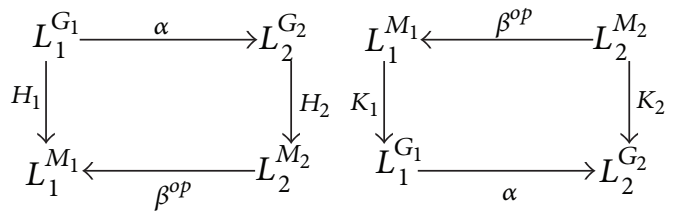

commute.

It should be emphasized that Definition 36 is inspired by the respective one of [29], which, in its turn, comes from the definition of the category GAL of order-preserving Galois connections of [32]. Moreover, two important features of the category S-FC ${ }^{D M R}$ are worth mentioning. Firstly, it never depends on the morphisms of the category $\mathbf{S}$, mentioned at the beginning of its definition (S-objects, however, are employed), which is reflected well enough in its truncated ground category. Secondly, its underlying functor to the ground category Set $\times$ Set $^{o p}$ is defined on objects as $|\mathscr{K}|=$ $\left(L^{G}, L^{M}\right)$, which is a huge difference from the already introduced categories of formal contexts (based on the machinery of topological systems), the underlying functor of which is just $|\mathscr{K}|=(G, M, L)$.

By analogy with the already presented categories of lattice-valued formal contexts, we introduce another version of the category of Definition 36. Unlike the case of the categories $\mathbf{S}-\mathbf{F C}{ }^{C}$ and $\mathbf{S}-\mathbf{F C}^{G W}$, where the change concerns the direction of the algebraic part of their morphisms, this time we change the set-theoretic morphism part and, therefore, use the notation " $(-)_{a}$ " ("alternative") instead of "(-) $)_{m}$ " ("modified").

Definition 37. Given a variety $\mathbf{L}$, which extends Quant, and a subcategory $\mathbf{S}$ of $\mathbf{L}, \mathbf{S}-\mathbf{F} \mathbf{C}_{a}^{D M R}$ is the category, concrete over the product category Set $\times$ Set, which comprises the following data.

Objects. Lattice-valued formal contexts $\mathscr{K}$ (in the sense of Definition 31) such that $L$ is an object of $\mathbf{S}$.

Morphisms. $\mathscr{K}_{1} \stackrel{f=(\alpha, \beta)}{\longrightarrow} \mathscr{K}_{2}$ are Set $\times$ Set-morphisms $\left(L_{1}^{G_{1}}, L_{1}^{M_{1}}\right) \stackrel{(\alpha, \beta)}{\longrightarrow}\left(L_{2}^{G_{2}}, L_{2}^{M_{2}}\right)$, which make the diagrams

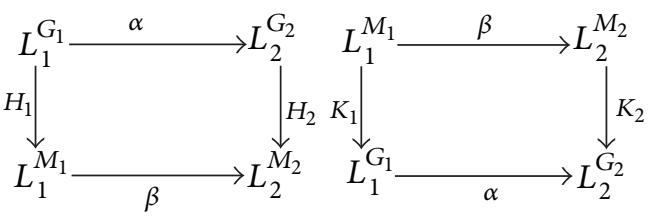

commute.

In the following, we adapt the technique of Theorem 28, to single out the possible isomorphic subcategories of the categories S-FC $^{D M R}$ and $\mathbf{S}-\mathbf{F C}_{a}^{D M R}$. 
Definition 38. $\mathbf{S}^{-} \mathbf{F C}_{i}^{D M R}$ (resp., S-FC $\mathbf{C}_{a i}^{D M R}$ ) is the nonfull subcategory of the category $\mathbf{S}$-FC ${ }^{D M R}$ (resp., S-FC $\mathbf{C}_{a}^{D M R}$ ), which has the same objects and whose morphisms $\mathscr{K}_{1} \stackrel{f}{\rightarrow} \mathscr{K}_{2}$ are such that $L_{2}^{M_{2}} \stackrel{\beta^{o p}}{\longrightarrow} L_{1}^{M_{1}}$ (resp., $\left.L_{1}^{M_{1}} \stackrel{\beta}{\rightarrow} L_{2}^{M_{2}}\right)$ is an isomorphism in Set, that is, bijective.

Theorem 39. There exists the isomorphism $\mathbf{S}-\mathbf{F C}_{i}^{D M R} \stackrel{F}{\rightarrow}$ S-FC $C_{a i}^{D M R}$, which is defined by the formula $F\left(\mathscr{K}_{1} \stackrel{f}{\rightarrow} \mathscr{K}_{2}\right)=$ $\mathscr{K}_{1} \stackrel{\left(\alpha,\left(\beta^{o p}\right)^{-1}\right)}{\longrightarrow} \mathscr{K}_{2}$.

The reader should be aware that the fixed-basis approach (i.e., the case of $\mathbf{S}=\mathbf{S}_{L}$ ) will (in general) provide nonisomorphic categories $L$-FC ${ }^{D M R}$ and $L-\mathbf{F C}_{a}^{D M R}$, since both of them are not dependant on $\mathbf{S}$-morphisms. More precisely, it is possible to obtain the following result.

Theorem 40. Suppose that $L$ is not a singleton, and, additionally, its quantale multiplication is not a constant map to $\perp_{L}$. Then the categories $L-\mathrm{FC}^{D M R}$ and $L-\mathrm{FC}_{a}^{D M R}$ are nonisomorphic.

Proof. It is easy to see that the context $\mathscr{K}=(X, X, I)$, where $X$ is the empty set and $X \times X \stackrel{I}{\rightarrow} L$ is the unique possible map, provides a terminal object in the category $L-F_{a}^{D M R}$. The category $L-F^{D M R}$, however, does not have a terminal object, which can be shown as follows.

Suppose the context $\mathscr{K}_{T}=\left(G_{T}, M_{T}, I_{T}\right)$ is terminal in $L$-FC ${ }^{D M R}$. Define a context $\mathscr{K}^{\prime}=\left(X^{\prime}, X^{\prime}, I^{\prime}\right)$, where $X^{\prime}$ is a nonempty set and $I^{\prime}=\underline{\mathrm{T}_{L}}$ (the constant map with value $\mathrm{T}_{L}$ ). One gets two equal constant maps $L^{X^{\prime}} \stackrel{H^{\prime}=K^{\prime}=T_{L}}{\longrightarrow} L^{X^{\prime}}$. Since there exists an $L$-FC ${ }^{D M R}$-morphism $\mathscr{K}^{\prime} \stackrel{f=(\alpha, \beta)}{\longrightarrow} \mathscr{K}_{T}$, the diagrams

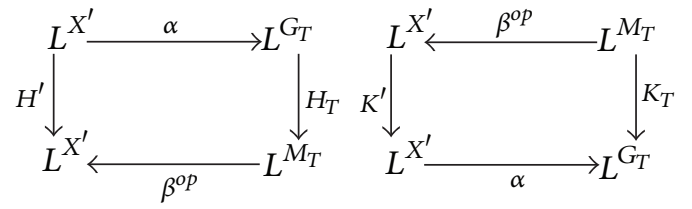

commute. It follows that the map $K_{T}$ in the right-hand diagram is constant (since $K^{\prime}$ has that property). Thus, if $L^{M_{T}} \stackrel{\beta^{\prime o p}}{\longrightarrow} L^{X^{\prime}}$ maps everything to $T_{L}$, then every map $L^{X^{\prime}} \stackrel{\alpha^{\prime}}{\longrightarrow}$ $L^{G_{T}}$, taking $T_{L}$ to the constant value of the map $K_{T}$, gives another $L$-FC $\bar{C}^{D M R}$-morphism $\mathscr{K}^{\prime} \stackrel{f^{\prime}}{\rightarrow} \mathscr{K}_{T}$. The uniqueness of such morphism necessitates $L^{G_{T}}$ to be a singleton, and then $G_{T}$ is the empty set (since $L$ is not a singleton). As a consequence, we get that the map $H_{T}$ is constant as well (with the value $T_{L}$ ). Define now another context $\mathscr{K}^{\prime \prime}=$ $\left(X^{\prime \prime}, X^{\prime \prime}, I^{\prime \prime}\right)$, where $X^{\prime \prime}$ is a nonempty set and $I^{\prime \prime}=\underline{\perp_{L}}$. Since the quantale multiplication of $L$ is not the constant map to $\perp_{L}$, we get a nonconstant map $H^{\prime \prime}\left(H^{\prime \prime}\left(\perp_{L}\right)=\underline{T}_{L} \neq H^{\prime \prime}\left(\underline{T_{L}}\right)\right)$, and, therefore, a contradiction, since the left-hand side of the previous two diagrams factorizes then (with the help of some $L$-FC ${ }^{D M R}$-morphism $\mathscr{K}^{\prime \prime} \stackrel{f^{\prime \prime}}{\longrightarrow} \mathscr{K}_{T}$ ) the nonconstant map $H^{\prime \prime}$ through the constant map $H_{T}$.

The case when $L$ is a singleton clearly provides the isomorphic categories $L-F_{C} C^{D M R}$ and $L-F_{C} D M R$. The case when $L$ is equipped with the constant $\perp_{L}$-valued quantale multiplication results in all context maps $H, K$ being constant (with the value $T_{L}$, which should additionally be preserved by the corresponding maps $\alpha$ and $\beta^{o p}$ (resp., $\beta$ )), which does not fit the machinery of Theorem 40 , and will be treated in our subsequent articles on lattice-valued FCA. We should emphasize, however, immediately that the just mentioned two cases are not that interesting (i.e., are usually skipped by the researchers), and, therefore, we can rightly conclude that the approaches of Definitions 36 and 37 are fundamentally different, which provides a (partial) justification for our alternative setting of the category $L-\mathrm{FC}_{a}^{D M R}$.

The difference between the categories S-FC ${ }^{D M R}$ and $\mathbf{S}-\mathbf{F C} \mathbf{C}_{a}^{D M R}$ is similar to that between the categories $\mathbf{S}-\mathbf{F C}^{C}$ and S-FC ${ }^{G W}$ (which served as our main motivation for introducing the approach of $\mathbf{S}-\mathbf{F C}_{a}^{D M R}$, which was mentioned, but never studied, in [29]).

4.4. Some Properties of Lattice-Valued Birkhoff Operators. It is a well-known (and easy to establish) fact that all orderreversing Galois connections between crisp powersets arise from a pair of crisp Birkhoff operators of Example 34. In the following, we show that the lattice-valued case destroys partly this essential property (the reader is advised to recall the notational remarks concerning particular maps of the powerset algebras, mentioned at the beginning of Section 2.2).

Theorem 41. If $\mathbf{L}$ extends $\mathbf{U Q u a n t}$, then every lattice-valued context $\mathscr{K}$ satisfies the following.

(1) $\left(H\left(\chi_{\{g\}}^{1_{L}}\right)\right)(m)=\left(K\left(\chi_{\{m\}}^{1_{L}}\right)\right)(g)$ for every $g \in G$ and every $m \in M$.

(2) $\left(H\left(\underline{a} \otimes \chi_{\{g\}}^{1_{L}}\right)\right)(m)=a \rightarrow{ }_{l}\left(H\left(\chi_{\{g\}}^{1_{L}}\right)\right)(m)$ for every $g \in G$, $m \in M$ and $a \in L$.

(3) $\left(K\left(\chi_{\{m\}}^{1_{L}} \otimes \underline{a}\right)\right)(g)=a \rightarrow{ }_{r}\left(K\left(\chi_{\{m\}}^{1_{L}}\right)\right)(g)$ for every $g \in G$, $m \in M$ and $a \in L$.

The previously mentioned items are equivalent to the following ones.

(a) $\left(H\left(\underline{a} \otimes \chi_{\{g\}}^{1_{L}}\right)\right)(m)=a \rightarrow{ }_{l}\left(K\left(\chi_{\{m\}}^{1_{L}}\right)\right)(g)$ for every $g \in G$, $m \in M$ and $a \in L$.

(b) $\left(K\left(\chi_{\{m\}}^{1_{L}} \otimes \underline{a}\right)\right)(g)=a \rightarrow{ }_{r}\left(H\left(\chi_{\{g\}}^{1_{L}}\right)\right)(m)$ for every $g \in G$, $m \in M$ and $a \in L$.

Proof. Ad (1). The item follows from the fact that $\left(H\left(\chi_{\{g\}}^{1_{L}}\right)\right)(m)=\bigwedge_{g^{\prime} \in G}\left(\chi_{\{g\}}^{1_{L}}\left(g^{\prime}\right) \rightarrow_{l} I\left(g^{\prime}, m\right)\right) \stackrel{(\dagger)}{=} 1_{L} \rightarrow_{l}$ $I(g, m)=I(g, m)=1_{L} \rightarrow{ }_{r} I(g, m) \stackrel{(\dagger)}{=} \bigwedge_{m^{\prime} \in M}\left(\chi_{\{m\}}^{1_{L}}\left(m^{\prime}\right) \rightarrow_{r}\right.$ $\left.I\left(g, m^{\prime}\right)\right)=\left(K\left(\chi_{\{m\}}^{1_{L}}\right)\right)(g)$, where $(\dagger)$ uses Theorem 6(2). 
$A d$ (2). $\left(H\left(\underline{a} \otimes \chi_{\{g\}}^{1_{L}}\right)\right)(m)=\bigwedge_{g^{\prime} \in G}\left(\left(\underline{a} \otimes \chi_{\{g\}}^{1_{L}}\right)\left(g^{\prime}\right) \rightarrow_{l}\right.$ $\left.I\left(g^{\prime}, m\right)\right)=\bigwedge_{g^{\prime} \in G}\left(\left(a \otimes \chi_{\{g\}}^{1_{L}}\left(g^{\prime}\right)\right) \rightarrow_{l} I\left(g^{\prime}, m\right)\right) \stackrel{(\dagger)}{=} \bigwedge_{g^{\prime} \in G}\left(a \rightarrow_{l}\right.$ $\left.\left(\chi_{\{g\}}^{1_{L}}\left(g^{\prime}\right) \rightarrow_{l} I\left(g^{\prime}, m\right)\right)\right) \quad \stackrel{(\dagger \dagger)}{=} \quad a \rightarrow_{l}\left(\bigwedge_{g^{\prime} \in G}\left(\chi_{\{g\}}^{1_{L}}\left(g^{\prime}\right) \rightarrow_{l}\right.\right.$ $\left.\left.I\left(g^{\prime}, m\right)\right)\right)=a \rightarrow{ }_{l}\left(H\left(\chi_{\{g\}}^{1_{L}}\right)\right)(m)$, where $(\dagger)$ (resp., $\left.(\dagger \dagger)\right)$ uses item (4) (resp., item (1)) of Theorem 6.

Ad (3). $\left(K\left(\chi_{\{m\}}^{1_{L}} \otimes \underline{a}\right)\right)(g)=\bigwedge_{m^{\prime} \in M}\left(\left(\chi_{\{m\}}^{1_{L}} \otimes \underline{a}\right)\left(m^{\prime}\right) \rightarrow_{r}\right.$ $\left.I\left(g, m^{\prime}\right)\right)=\bigwedge_{m^{\prime} \in M}\left(\left(\chi_{\{m\}}^{1_{L}}\left(m^{\prime}\right) \otimes a\right) \rightarrow_{r} I\left(g, m^{\prime}\right)\right) \stackrel{(\dagger)}{=}$ $\bigwedge_{m^{\prime} \in M}\left(a \rightarrow{ }_{r}\left(\chi_{\{m\}}^{1_{L}}\left(m^{\prime}\right) \rightarrow_{r} I\left(g, m^{\prime}\right)\right)\right) \quad \stackrel{(\dagger \dagger)}{=} \quad a \rightarrow_{r}$ $\left(\bigwedge_{m^{\prime} \in M}\left(\chi_{\{m\}}^{1_{L}}\left(m^{\prime}\right) \rightarrow{ }_{r} I\left(g, m^{\prime}\right)\right)\right)=a \rightarrow{ }_{r}\left(K\left(\chi_{\{m\}}^{1_{L}}\right)\right)(g)$, where $(\dagger)$ (resp., $(\dagger \dagger))$ uses item (4) (resp., item (1)) of Theorem 6.

Ad (1), (2), (3) $\Rightarrow(a),(b)$. Use (1) in the right-hand side of both (2) and (3).

$A d(a),(b) \Rightarrow(1),(2),(3)$. Put $a=1_{L}$ in any of $(a)$ or $(b)$ and get (1). Now, use (1) in the right-hand side of both $(a)$ and (b), to get (2) and (3), respectively.

Theorem 41 paves the way for a partial generalization of the previously mentioned result on generating orderreversing Galois connections between powersets.

Theorem 42. Let $G, M$ be sets and let $L$ be a unital quantale. For every order-reversing Galois connection $\left(L^{G}, \alpha, \beta, L^{M}\right)$, the following statements are equivalent.

(1) There exists a map $G \times M \stackrel{I}{\rightarrow} L$ such that $\alpha=H$ and $\beta=K$.

(2) (a) $\left(\alpha\left(\chi_{\{g\}}^{1_{L}}\right)\right)(m)=\left(\beta\left(\chi_{\{m\}}^{1_{L}}\right)\right)(g)$ for every $g \in G$ and every $m \in M$.

(b) $\left(\alpha\left(\underline{a} \otimes \chi_{\{g\}}^{1_{L}}\right)\right)(m)=a \rightarrow{ }_{l}\left(\alpha\left(\chi_{\{g\}}^{1_{L}}\right)\right)(m)$ for every $g \in G, m \in M$ and $a \in L$.

(c) $\left(\beta\left(\chi_{\{m\}}^{1_{L}} \otimes \underline{a}\right)\right)(g)=a \rightarrow{ }_{r}\left(\beta\left(\chi_{\{m\}}^{1_{L}}\right)\right)(g)$ for every $g \in G, m \in M$ and $a \in L$.

(3) (a) $\left(\alpha\left(\underline{a} \otimes \chi_{\{g\}}^{1_{L}}\right)\right)(m)=a \rightarrow{ }_{l}\left(\beta\left(\chi_{\{m\}}^{1_{L}}\right)\right)(g)$ for every $g \in G, m \in M$ and $a \in L$.

(b) $\left(\beta\left(\chi_{\{m\}}^{1_{L}} \otimes \underline{a}\right)\right)(g)=a \rightarrow{ }_{r}\left(\alpha\left(\chi_{\{g\}}^{1_{L}}\right)\right)(m)$ for every $g \in G, m \in M$ and $a \in L$.

Proof. (1) $\Rightarrow$ (2) and (2) $\Leftrightarrow$ (3). It follows from Theorem 41 .

$(2) \Rightarrow(1)$. Define the required map $G \times M \stackrel{I}{\rightarrow} L$ by $I(g, m)=\left(\alpha\left(\chi_{\{g\}}^{1_{L}}\right)\right)(m) \stackrel{(a)}{=}\left(\beta\left(\chi_{\{m\}}^{1_{L}}\right)\right)(g)$. Given $s \in L^{G}$ and $m \in M$, it follows that $(H(s))(m)=\bigwedge_{g \in G}\left(s(g) \rightarrow{ }_{l} I(g, m)\right)=$ $\left.\bigwedge_{g \in G}\left(s(g) \rightarrow{ }_{l}\left(\alpha\left(\chi_{\{g\}}^{1_{L}}\right)\right)(m)\right) \stackrel{(b)}{=} \bigwedge_{g \in G}\left(\alpha(s(g)) \otimes \chi_{\{g\}}^{1_{L}}\right)\right)(m) \stackrel{(\dagger)}{=}$ $\left(\alpha\left(\bigvee_{g \in G}\left(s(g) \otimes \chi_{\{g\}}^{1_{L}}\right)\right)\right)(m)=(\alpha(s))(m)$ (notice that $\left(\bigvee_{g \in G}\left(s\left(\underline{g)} \otimes \chi_{\{g\}}^{1_{L}}\right)\right)\left(g^{\prime}\right)=\bigvee_{g \in G} \underline{(s(g)}\left(g^{\prime}\right) \otimes \chi_{\{g\}}^{1_{L}}\left(g^{\prime}\right)\right)=s\left(g^{\prime}\right) \otimes$ $1_{L}=s\left(g^{\prime}\right)$ for every $\left.g^{\prime} \in G\right)$, whereas, given $t \in L^{M}$ and $g \in G$, it follows that $(K(t))(g)=\bigwedge_{m \in M}\left(t(m) \rightarrow{ }_{r} I(g, m)\right)=$ $\bigwedge_{m \in M}\left(t(m) \rightarrow{ }_{r}\left(\beta\left(\chi_{\{m\}}^{1_{L}}\right)\right)(g)\right) \stackrel{(c)}{=} \bigwedge_{m \in M}\left(\beta\left(\chi_{\{m\}}^{1_{L}} \otimes t \underline{t(m)}\right)\right)(g) \stackrel{(\dagger)}{=}$ $\left(\beta\left(\bigvee_{m \in M}\left(\chi_{\{m\}}^{1_{L}} \otimes \underline{t(m)}\right)\right)\right)(g)=(\beta(t))(g)$, where $(\dagger)$ uses Theorem 19(3).
Theorems 41 and 42 show that in the lattice-valued setting, every map $G \times M \stackrel{I}{\rightarrow} L$ provides an order-reversing Galois connection, whereas the converse way is possible under certain restrictions only. As a simple counterexam-

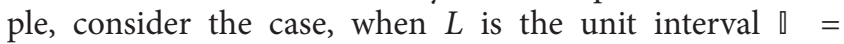
$([0,1], \bigvee, \bigwedge, 1)$, and both $G$ and $M$ are singletons. We can,

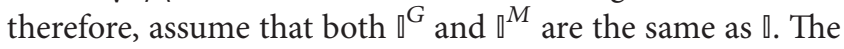

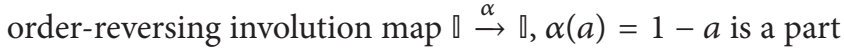
of the order-reversing Galois connection $(\square, \alpha, \alpha, \llbracket)$. It is easy to see that the condition of, for example, Theorem 42(3)(a) amounts to $\alpha(a)=a \rightarrow \alpha(1)$ for every $a \in \mathbb{Q}$ (notice that the quantale in question is commutative). However, for $a=1 / 2$, we obtain that $\alpha(1 / 2)=1 / 2 \neq 0=1 / 2 \rightarrow 0=1 / 2 \rightarrow \alpha(1)$.

We also would like to emphasize here that [40] uses a different approach to Galois connections (called polarities of certain types), which has the advantage of still preserving the one-to-one correspondence between them and Birkhoff operators even in the lattice-valued case [40, Theorem 3.11]. The underlying framework of such polarities, however, is more demanding than that of this paper (e.g., requires enriched categories) and, therefore, will be not considered here. Also notice that [64, Proposition 7.1] provides an analogue of Theorem 42 for the case of strictly two-sided commutative quantales, considering, however, lattice-valued versions of the powerset operators generated by a relation and extending those given at the beginning of Section 2.2 and, therefore, dealing with order-preserving Galois connections. More precisely, in the notation of [64], every formal context $\mathscr{K}=(G, M, L, I)$ (where $L$ is additionally assumed to be a strictly two-sided commutative quantale) provides the following generalizations of the standard powerset operators:

(1) $L^{G} \stackrel{I^{\otimes}}{\rightarrow} L^{M}$ given by $\left(I^{\otimes}(s)\right)(m)=\bigvee_{g \in G} I(g, m) \otimes s(g)$ (forward powerset operator),

(2) $L^{M} \stackrel{I_{-}^{\rightarrow}}{\longrightarrow} L^{G}$ given by $\left(I_{-}^{\rightarrow}(t)\right)(g)=\bigwedge_{m \in M} I(g, m) \rightarrow$ $t(m)$ (backward powerset operator).

In particular, $\left(L^{G}, I^{\otimes}, I_{-}^{\rightarrow}, L^{M}\right)$ is an order-preserving Galois connection (recall Definition 18). It is the main purpose of [64, Proposition 7.1] to clarify the conditions, when an orderpreserving Galois connection $\left(L^{G}, \alpha, \beta, L^{M}\right)$ has the previous form, that is, is generated by a map $G \times M \stackrel{I}{\rightarrow} L$.

We end the subsection with another simple (but useful) property of lattice-valued Birkhoff operators.

Lemma 43. Given a lattice-valued formal context $\mathscr{K}$ such that $I=\underline{\mathrm{T}_{L}}$, it follows that $H=\underline{\underline{\mathrm{T}_{L}}}$ and $K=\underline{\underline{\mathrm{T}_{L}}}$. In particular, in the crisp case, $H=\underline{M}$ and $\overline{\overline{K=}} \underline{G}$.

Proof. It is enough to consider the case of $H$ (that of $K$ being similar). Given $s \in L^{G}$, for every $m \in M,(H(s))(m)=$ $\bigwedge_{g \in G}\left(s(g) \rightarrow{ }_{l} I(g, m)\right)=\bigwedge_{g \in G}\left(s(g) \rightarrow{ }_{l} \top_{L}\right)=\bigwedge_{g \in G} \top_{L}=$ $\mathrm{T}_{L}$, that is, $H(s)=\underline{\mathrm{T}_{L}}$.

4.5. Lattice-Valued Formal Concepts, Protoconcepts, and Preconcepts. In the developments of [29], Denniston et al. use 
lattice-valued extensions of the notions of formal concept, protoconcept, and preconcept of, for example, [36]. In the following, we recall their respective definitions and show some of their properties in our more general framework, which will be used later on in the paper.

Definition 44. Let $\mathscr{K}$ be a lattice-valued formal context and let $s \in L^{G}, t \in L^{M}$. The pair $(s, t)$ is called a

(1) (lattice-valued) formal concept of $\mathscr{K}$ provided that $H(s)=t$ and $K(t)=s$,

(2) (lattice-valued) formal protoconcept of $\mathscr{K}$ provided that $K \circ H(s)=K(t)$ (equivalently, $H \circ K(t)=H(s)$ ),

(3) (lattice-valued) formal preconcept of $\mathscr{K}$ provided that $s \leqslant K(t)$ (equivalently, $t \leqslant H(s)$ ).

Using the properties of Galois connections, mentioned in Theorem 19, one can easily get that every concept is a protoconcept, and every protoconcept is a preconcept, whereas the converse implications, in general, are not true. Moreover, the next result provides simple properties of the notions of Definition 44 with respect to the morphisms of the previously mentioned categories S-FC ${ }^{D M R}$ and $\mathbf{S}-\mathbf{F C}_{a}^{D M R}$.

Theorem 45. Let $\mathscr{K}_{1} \stackrel{f}{\rightarrow} \mathscr{K}_{2}$ be a morphism of the category S-FC ${ }^{D M R}$ and let $s_{1} \in L_{1}^{G_{1}}, t_{2} \in L_{2}^{M_{2}}$.

(1) $\left(s_{1}, \beta^{o p}\left(t_{2}\right)\right)$ is a formal concept of $\mathscr{K}_{1}$ and $H_{2} \circ K_{2}\left(t_{2}\right)=$ $t_{2}$ iff $\left(\alpha\left(s_{1}\right), t_{2}\right)$ is a formal concept of $\mathscr{K}_{2}$ and $K_{1}$ 。 $H_{1}\left(s_{1}\right)=s_{1}$.

(2) If both $L_{1}^{G_{1}} \stackrel{\alpha}{\rightarrow} L_{2}^{G_{2}}$ and $L_{2}^{M_{2}} \stackrel{\beta^{o p}}{\longrightarrow} L_{1}^{M_{1}}$ reflect fixed points, that is, $K_{2} \circ H_{2} \circ \alpha(s)=\alpha(s)$ implies $K_{1} \circ H_{1}(s)=$ $s$ for every $s \in L_{1}^{G_{1}}$, and $H_{1} \circ K_{1} \circ \beta^{o p}(t)=\beta^{o p}(t)$ implies $H_{2} \circ K_{2}(t)=t$ for every $t \in L_{2}^{M_{2}}$, then $\left(s_{1}, \beta^{o p}\left(t_{2}\right)\right)$ is a formal concept of $\mathscr{K}_{1}$ iff $\left(\alpha\left(s_{1}\right), t_{2}\right)$ is a formal concept of $\mathscr{K}_{2}$.

(3) If both $L_{1}^{G_{1}} \stackrel{\alpha}{\rightarrow} L_{2}^{G_{2}}$ and $L_{2}^{M_{2}} \stackrel{\beta^{o p}}{\longrightarrow} L_{1}^{M_{1}}$ are injective, then $\left(s_{1}, \beta^{o p}\left(t_{2}\right)\right)$ is a formal concept of $\mathscr{K}_{1}$ iff $\left(\alpha\left(s_{1}\right), t_{2}\right)$ is a formal concept of $\mathscr{K}_{2}$.

(4) $\left(s_{1}, \beta^{o p}\left(t_{2}\right)\right)$ is a formal protoconcept of $\mathscr{K}_{1}$ iff $\left(\alpha\left(s_{1}\right), t_{2}\right)$ is a formal protoconcept of $\mathscr{K}_{2}$.

(5) If both $L_{1}^{G_{1}} \stackrel{\alpha}{\rightarrow} L_{2}^{G_{2}}$ and $L_{2}^{M_{2}} \stackrel{\beta^{o p}}{\longrightarrow} L_{1}^{M_{1}}$ are orderpreserving, then $\left(s_{1}, \beta^{o p}\left(t_{2}\right)\right)$ is a formal preconcept of $\mathscr{K}_{1}$ iff $\left(\alpha\left(s_{1}\right), t_{2}\right)$ is a formal preconcept of $\mathscr{K}_{2}$.

If $L_{1}^{G_{1}} \stackrel{\alpha}{\rightarrow} L_{2}^{G_{2}}$ (resp., $L_{2}^{M_{2}} \stackrel{\beta^{o p}}{\longrightarrow} L_{1}^{M_{1}}$ ) is injective, then $\alpha$ (resp., $\beta$ ) reflects fixed points in the sense of (2).

Proof. Ad (1). “ $\Rightarrow$ ": Since both $s_{1}=K_{1} \circ \beta^{\circ p}\left(t_{2}\right)$ and $H_{1}\left(s_{1}\right)=$ $\beta^{o p}\left(t_{2}\right)$, we get $\alpha\left(s_{1}\right)=\alpha \circ K_{1} \circ \beta^{o p}\left(t_{2}\right)=K_{2}\left(t_{2}\right), H_{2} \circ \alpha\left(s_{1}\right)=$ $H_{2} \circ \alpha \circ K_{1} \circ \beta^{\circ p}\left(t_{2}\right)=H_{2} \circ K_{2}\left(t_{2}\right)=t_{2}$, and, moreover, $K_{1} \circ H_{1}\left(s_{1}\right)=K_{1} \circ \beta^{\circ p}\left(t_{2}\right)=s_{1}$. "Е": Since both $H_{2} \circ \alpha\left(s_{1}\right)=t_{2}$ and $\alpha\left(s_{1}\right)=K_{2}\left(t_{2}\right)$, we get $\beta^{o p}\left(t_{2}\right)=\beta^{o p} \circ H_{2} \circ \alpha\left(s_{1}\right)=H_{1}\left(s_{1}\right)$, $K_{1} \circ \beta^{o p}\left(t_{2}\right)=K_{1} \circ \beta^{o p} \circ H_{2} \circ \alpha\left(s_{1}\right)=K_{1} \circ H_{1}\left(s_{1}\right)=s_{1}$, and, moreover, $H_{2} \circ K_{2}\left(t_{2}\right)=H_{2} \circ \alpha\left(s_{1}\right)=t_{2}$.
Ad (2). “ $\Rightarrow ": \beta^{o p}\left(t_{2}\right)=H_{1}\left(s_{1}\right)$ yields $H_{1} \circ K_{1} \circ \beta^{o p}\left(t_{2}\right)=H_{1} \circ$ $K_{1} \circ H_{1}\left(s_{1}\right)=H_{1}\left(s_{1}\right)=\beta^{o p}\left(t_{2}\right)$, and, therefore, $H_{2} \circ K_{2}\left(t_{2}\right)=$ $t_{2}$, since $\beta^{o p}$ reflects fixed points. Now, employ item (1). " $\Leftarrow$ ": $K_{2}\left(t_{2}\right)=\alpha\left(s_{1}\right)$ yields $K_{2} \circ H_{2} \circ \alpha\left(s_{1}\right)=K_{2} \circ H_{2} \circ K_{2}\left(t_{2}\right)=$ $K_{2}\left(t_{2}\right)=\alpha\left(s_{1}\right)$, and, therefore, $K_{1} \circ H_{1}\left(s_{1}\right)=s_{1}$, since $\alpha$ reflects fixed points. Now, employ item (1).

Ad (3). It follows from (2) and the last claim of the theorem.

Ad (4). “ $\Rightarrow$ ": Since $K_{1} \circ H_{1}\left(s_{1}\right)=K_{1} \circ \beta^{o p}\left(t_{2}\right)$, we get $K_{2} \circ H_{2} \circ \alpha\left(s_{1}\right)=\alpha \circ K_{1} \circ \beta^{\circ \circ} \circ H_{2} \circ \alpha\left(s_{1}\right)=\alpha \circ K_{1} \circ H_{1}\left(s_{1}\right)=$ $\alpha \circ K_{1} \circ \beta^{\circ p}\left(t_{2}\right)=K_{2}\left(t_{2}\right)$. “ $\Leftarrow$ ": Since $H_{2} \circ K_{2}\left(t_{2}\right)=H_{2} \circ \alpha\left(s_{1}\right)$, we get $H_{1} \circ K_{1} \circ \beta^{o p}\left(t_{2}\right)=\beta^{o p} \circ H_{2} \circ \alpha \circ K_{1} \circ \beta^{o p}\left(t_{2}\right)=\beta^{o p} \circ$ $H_{2} \circ K_{2}\left(t_{2}\right)=\beta^{\circ \circ} \circ H_{2} \circ \alpha\left(s_{1}\right)=H_{1}\left(s_{1}\right)$.

Ad (5). “ $\Rightarrow ": s_{1} \leqslant K_{1} \circ \beta^{o p}\left(t_{2}\right)$ implies $\alpha\left(s_{1}\right) \leqslant \alpha \circ K_{1} \circ$ $\beta^{o p}\left(t_{2}\right)=K_{2}\left(t_{2}\right)$ ( $\alpha$ is order-preserving). “ $\Leftarrow ": t_{2} \leqslant H_{2} \circ \alpha\left(s_{1}\right)$ yields $\beta^{o p}\left(t_{2}\right) \leqslant \beta^{o p} \circ H_{2} \circ \alpha\left(s_{1}\right)=H_{1}\left(s_{1}\right)\left(\beta^{o p}\right.$ is orderpreserving).

For the last claim, notice that given $s \in L_{1}^{G_{1}}$ such that $K_{2}$ 。 $H_{2} \circ \alpha(s)=\alpha(s)$, it follows that $\alpha \circ K_{1} \circ H_{1}(s)=\alpha \circ K_{1} \circ \beta^{\circ p} \circ H_{2} \circ$ $\alpha(s)=K_{2} \circ H_{2} \circ \alpha(s)=\alpha(s)$ and thus, $K_{1} \circ H_{1}(s)=s$ (injectivity of $\alpha$ ). Additionally, given $t \in L_{2}^{M_{2}}$ such that $H_{1} \circ K_{1} \circ \beta^{o p}(t)=$ $\beta^{o p}(t)$, it follows that $\beta^{o p} \circ H_{2} \circ K_{2}(t)=\beta^{o p} \circ H_{2} \circ \alpha \circ K_{1} \circ \beta^{o p}(t)=$ $H_{1} \circ K_{1} \circ \beta^{\circ p}(t)=\beta^{\circ p}(t)$ and thus, $H_{2} \circ K_{2}(t)=t$ (injectivity of $\left.\beta^{o p}\right)$.

Theorem 46. Let $\mathscr{K}_{1} \stackrel{f}{\rightarrow} \mathscr{K}_{2}$ be a morphism of the category S-FC ${ }_{a}^{D M R}$ and let $s_{1} \in L_{1}^{G_{1}}, t_{1} \in L_{1}^{M_{1}}$.

(1) If both $L_{1}^{G_{1}} \stackrel{\alpha}{\rightarrow} L_{2}^{G_{2}}$ and $L_{1}^{M_{1}} \stackrel{\beta}{\rightarrow} L_{2}^{M_{2}}$ are injective, then $\left(s_{1}, t_{1}\right)$ is a formal concept of $\mathscr{K}_{1}$ iff $\left(\alpha\left(s_{1}\right), \beta\left(t_{1}\right)\right)$ is a formal concept of $\mathscr{K}_{2}$.

(2) If one of $L_{1}^{G_{1}} \stackrel{\alpha}{\rightarrow} L_{2}^{G_{2}}, L_{1}^{M_{1}} \stackrel{\beta}{\rightarrow} L_{2}^{M_{2}}$ is injective, then $\left(s_{1}, t_{1}\right)$ is a formal protoconcept of $\mathscr{K}_{1}$ iff $\left(\alpha\left(s_{1}\right), \beta\left(t_{1}\right)\right)$ is a formal protoconcept of $\mathscr{K}_{2}$.

(3) If one of $L_{1}^{G_{1}} \stackrel{\alpha}{\rightarrow} L_{2}^{G_{2}}, L_{1}^{M_{1}} \stackrel{\beta}{\rightarrow} L_{2}^{M_{2}}$ is order-preserving and, moreover, one of the maps is order-reflecting, then $\left(s_{1}, t_{1}\right)$ is a formal preconcept of $\mathscr{K}_{1}$ iff $\left(\alpha\left(s_{1}\right), \beta\left(t_{1}\right)\right)$ is a formal preconcept of $\mathscr{K}_{2}$.

Proof. Ad (1). “ $\Rightarrow$ ": Since $H_{1}\left(s_{1}\right)=t_{1}$ and $K_{1}\left(t_{1}\right)=s_{1}, H_{2}$ 。 $\alpha\left(s_{1}\right)=\beta \circ H_{1}\left(s_{1}\right)=\beta\left(t_{1}\right)$ and $K_{2} \circ \beta\left(t_{1}\right)=\alpha \circ K_{1}\left(t_{1}\right)=\alpha\left(s_{1}\right)$ (no injectivity condition is employed). “ $\Leftarrow$ ": Since $H_{2} \circ \alpha\left(s_{1}\right)=$ $\beta\left(t_{1}\right)$ and $K_{2} \circ \beta\left(t_{1}\right)=\alpha\left(s_{1}\right), \beta \circ H_{1}\left(s_{1}\right)=H_{2} \circ \alpha\left(s_{1}\right)=\beta\left(t_{1}\right)$ and $\alpha \circ K_{1}\left(t_{1}\right)=K_{2} \circ \beta\left(t_{1}\right)=\alpha\left(s_{1}\right)$ imply $H_{1}\left(s_{1}\right)=t_{1}$ and $K_{1}\left(t_{1}\right)=s_{1}$, respectively (both $\alpha$ and $\beta$ are injective).

Ad (2). “ $\Rightarrow$ ": Since $K_{1} \circ H_{1}\left(s_{1}\right)=K_{1}\left(t_{1}\right), K_{2} \circ H_{2} \circ \alpha\left(s_{1}\right)=$ $K_{2} \circ \beta \circ H_{1}\left(s_{1}\right)=\alpha \circ K_{1} \circ H_{1}\left(s_{1}\right)=\alpha \circ K_{1}\left(t_{1}\right)=K_{2} \circ \beta\left(t_{1}\right)$ (no injectivity condition is employed). " $\Leftarrow$ ": If $L_{1}^{G_{1}} \stackrel{\alpha}{\rightarrow} L_{2}^{G_{2}}$ is injective, then $K_{2} \circ H_{2} \circ \alpha\left(s_{1}\right)=K_{2} \circ \beta\left(t_{1}\right)$ implies $\alpha \circ K_{1} \circ$ $H_{1}\left(s_{1}\right)=K_{2} \circ \beta \circ H_{1}\left(s_{1}\right)=K_{2} \circ H_{2} \circ \alpha\left(s_{1}\right)=K_{2} \circ \beta\left(t_{1}\right)=$ $\alpha \circ K_{1}\left(t_{1}\right)$ and thus, $K_{1} \circ H_{1}\left(s_{1}\right)=K_{1}\left(t_{1}\right)$. If $L_{1}^{M_{1}} \stackrel{\beta}{\rightarrow} L_{2}^{M_{2}}$ is injective, then $H_{2} \circ K_{2} \circ \beta\left(t_{1}\right)=H_{2} \circ \alpha\left(s_{1}\right)$ implies $\beta \circ H_{1} \circ$ $K_{1}\left(t_{1}\right)=H_{2} \circ \alpha \circ K_{1}\left(t_{1}\right)=H_{2} \circ K_{2} \circ \beta\left(t_{1}\right)=H_{2} \circ \alpha\left(s_{1}\right)=$ $\beta \circ H_{1}\left(s_{1}\right)$ and thus, $H_{1} \circ K_{1}\left(t_{1}\right)=H_{1}\left(s_{1}\right)$. 
Ad (3). “" $\Rightarrow$ : If $L_{1}^{G_{1}} \stackrel{\alpha}{\rightarrow} L_{2}^{G_{2}}$ is order-preserving, then $s_{1} \leqslant$ $K_{1}\left(t_{1}\right)$ implies $\alpha\left(s_{1}\right) \leqslant \alpha \circ K_{1}\left(t_{1}\right)=K_{2} \circ \beta\left(t_{1}\right)$. If $L_{1}^{M_{1}} \stackrel{\beta}{\rightarrow} L_{2}^{M_{2}}$ is order-preserving, then $t_{1} \leqslant H_{1}\left(s_{1}\right)$ yields $\beta\left(t_{1}\right) \leqslant \beta \circ H_{1}\left(s_{1}\right)=$ $H_{2} \circ \alpha\left(s_{1}\right)$. " $\models$ ": If $L_{1}^{G_{1}} \stackrel{\alpha}{\rightarrow} L_{2}^{G_{2}}$ is order-reflecting, then $\alpha\left(s_{1}\right) \leqslant$ $K_{2} \circ \beta\left(t_{1}\right)=\alpha \circ K_{1}\left(t_{1}\right)$ implies $s_{1} \leqslant K_{1}\left(t_{1}\right)$. If $L_{1}^{M_{1}} \stackrel{\beta}{\rightarrow} L_{2}^{M_{2}}$ is order-reflecting, then $\beta\left(t_{1}\right) \leqslant H_{2} \circ \alpha\left(s_{1}\right)=\beta \circ H_{1}\left(s_{1}\right)$ yields $t_{1} \leqslant H_{1}\left(s_{1}\right)$.

The two previous theorems provide crucial tools for defining certain functors between the categories of latticevalued formal contexts, which is the main topic of the next section.

\section{Functorial Relationships between the Categories of Lattice-Valued Formal Contexts}

The previous section introduced several categories (partly motivated by the respective ones of [29]), the objects of which are lattice-valued formal contexts. It is the purpose of this section to consider functorial relationships between the new categories, the functors in question being again partly inspired by the respective approach of [29]. As a result, we not only extend (the change from commutative quantales to noncommutative ones and the shift from fixed-basis to variable-basis) and streamline the machinery of Denniston et al., but also provide several new functors (induced by new categories), thereby clarifying the relationships between different frameworks for doing lattice-valued FCA and bringing to light their respective (dis)advantages.

5.1. S-FC ${ }^{C}$ and $\mathbf{S}-\mathbf{F C}_{m}^{C}$ versus $\mathbf{S}-\mathbf{F C}^{D M R}$ and $\mathbf{S}-\mathbf{F C}_{a}^{D M R}$. In this subsection, we consider possible functorial links between the categories $\mathbf{S}-\mathbf{F C} C^{C}$ and $\mathbf{S}-\mathbf{F C} C_{m}^{C}$, on one side, and the categories $\mathbf{S}-\mathbf{F C}{ }^{D M R}$ and $\mathbf{S}-\mathbf{F C}_{a}^{D M R}$, on the other.

5.1.1. From $\mathbf{S}-\mathbf{F C}_{m}^{C}$ to $\mathbf{S}-\mathbf{F C}_{a}^{D M R}$. In this subsection, we construct a functorial embedding of a particular subcategory of the category $\mathbf{S}-\mathbf{F} \mathbf{C}_{m}^{C}$ into the category $\mathbf{S}-\mathbf{F} \mathbf{C}_{a}^{D M R}$. We begin with singling out the subcategory in question.

Definition 47. $\mathbf{S}-\mathbf{F C}_{m *}^{C}$ is the (nonfull) subcategory of the category $\mathbf{S}-\mathbf{F C}_{m}^{C}$, with the same objects, whose morphisms $\mathscr{K}_{1} \stackrel{f}{\rightarrow} \mathscr{K}_{2}$ are such that the maps $G_{1} \stackrel{\alpha}{\rightarrow} G_{2}, M_{2} \stackrel{\beta^{o p}}{\rightarrow} M_{1}$ are surjective, whereas the $\mathbf{S}$-morphism $L_{1} \stackrel{\varphi}{\rightarrow} L_{2}$ preserves $\bigwedge, \rightarrow_{l}$ and $\rightarrow{ }_{r}$.

Notice that the notation $(-)_{*}$ will usually be used for the constructed subcategories in this paper, the scope of each $(-)_{*}$ being limited to its respective subsection. More important constructions, however, will be distinguished, respectively.

With the category of Definition 47 in hand, we can construct the following functor (the reader is advised to recall our powerset operator notations from Section 2.2).
Theorem 48. There exists the functor $\mathbf{S}-\mathbf{F C}_{m *}^{C} \stackrel{H_{C m D a}}{\longrightarrow}$ S-FC ${ }_{a}^{D M R}$, which is defined by the formula $H_{C m D a}\left(\mathscr{K}_{1} \stackrel{f}{\rightarrow}\right.$ $\left.\mathscr{K}_{2}\right)=\mathscr{K}_{1} \stackrel{\left((\alpha, \varphi)^{\rightarrow},(\beta, \varphi)^{\leftarrow o}\right)}{\longrightarrow} \mathscr{K}_{2}$.

Proof. To show that the functor is correct on morphisms, we verify commutativity of the diagrams

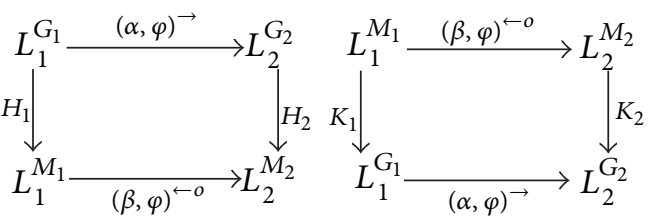

For the left-hand diagram, notice that given $s \in L_{1}^{G_{1}}$ and $m_{2} \in M_{2}$,

$$
\begin{aligned}
& \left(H_{2} \circ(\alpha, \varphi) \rightarrow(s)\right)\left(m_{2}\right) \\
& =\bigwedge_{g_{2} \in G_{2}}\left(((\alpha, \varphi) \rightarrow(s))\left(g_{2}\right) \longrightarrow_{l} I_{2}\left(g_{2}, m_{2}\right)\right) \\
& =\bigwedge_{g_{2} \in G_{2}}\left(\varphi\left(\bigvee_{\alpha\left(g_{1}\right)=g_{2}} s\left(g_{1}\right)\right) \longrightarrow_{l} I_{2}\left(g_{2}, m_{2}\right)\right) \\
& =\bigwedge_{g_{2} \in G_{2}}\left(\left(\bigvee_{\alpha\left(g_{1}\right)=g_{2}} \varphi \circ s\left(g_{1}\right)\right) \longrightarrow_{l} I_{2}\left(g_{2}, m_{2}\right)\right) \\
& \stackrel{(\dagger)}{=} \bigwedge_{g_{2} \in G_{2}} \bigwedge_{\alpha\left(g_{1}\right)=g_{2}}\left(\varphi \circ s\left(g_{1}\right) \longrightarrow I_{2}\left(g_{2}, m_{2}\right)\right) \\
& =\bigwedge_{g_{1} \in G_{1}}\left(\varphi \circ s\left(g_{1}\right) \longrightarrow_{l} I_{2}\left(\alpha\left(g_{1}\right), m_{2}\right)\right) \\
& \stackrel{(\dagger \dagger)}{=} \bigwedge_{g_{1} \in G_{1}}\left(\varphi \circ s\left(g_{1}\right) \longrightarrow_{l} \varphi \circ I_{1}\left(g_{1}, \beta^{o p}\left(m_{2}\right)\right)\right) \\
& \stackrel{(\dagger+\dagger)}{=} \varphi\left(\bigwedge_{g_{1} \in G_{1}}\left(s\left(g_{1}\right) \longrightarrow_{l} I_{1}\left(g_{1}, \beta^{o p}\left(m_{2}\right)\right)\right)\right) \\
& =\varphi \circ\left(H_{1}(s)\right)\left(\beta^{o p}\left(m_{2}\right)\right) \\
& =\left((\beta, \varphi)^{\leftarrow o} \circ H_{1}(s)\right)\left(m_{2}\right),
\end{aligned}
$$

where $(\dagger)$ relies on Theorem $6(2)$, $(\dagger \dagger)$ uses the fact that $\mathscr{K}_{1} \stackrel{f}{\rightarrow} \mathscr{K}_{2}$ is a morphism of $\mathbf{S}-\mathbf{F C}_{m}^{C}$, whereas $(\dagger \dagger \dagger)$ employs the definition of the category $\mathbf{S}-\mathbf{F C}_{m *}^{C}$. 
For the right-hand diagram, notice that given $t \in L_{1}^{M_{1}}$ and $g_{2} \in G_{2}$,

$$
\begin{aligned}
& \left((\alpha, \varphi) \rightarrow \circ K_{1}(t)\right)\left(g_{2}\right) \\
& =\varphi\left(\bigvee_{\alpha\left(g_{1}\right)=g_{2}}\left(K_{1}(t)\right)\left(g_{1}\right)\right) \\
& =\varphi\left(\bigvee _ { \alpha ( g _ { 1 } ) = g _ { 2 } } \bigwedge _ { m _ { 1 } \in M _ { 1 } } \left(t\left(m_{1}\right)\right.\right. \\
& \left.\left.\longrightarrow_{r} I_{1}\left(g_{1}, m_{1}\right)\right)\right) \\
& \stackrel{(\dagger)}{=} \varphi\left(\bigvee _ { \alpha ( g _ { 1 } ) = g _ { 2 } } \bigwedge _ { m _ { 2 } \in M _ { 2 } } \left(t \circ \beta^{o p}\left(m_{2}\right)\right.\right. \\
& \left.\left.\longrightarrow_{r} I_{1}\left(g_{1}, \beta^{o p}\left(m_{2}\right)\right)\right)\right) \\
& \begin{aligned}
& \stackrel{(+\dagger)}{=} \bigvee_{\alpha\left(g_{1}\right)=g_{2}} \bigwedge_{m_{2} \in M_{2}}\left(\varphi \circ t \circ \beta^{o p}\left(m_{2}\right)\right. \\
&\left.\longrightarrow r \varphi \circ I_{1}\left(g_{1}, \beta^{o p}\left(m_{2}\right)\right)\right)
\end{aligned} \\
& \stackrel{(\dagger \dagger \dagger)}{=} \bigvee_{\alpha\left(g_{1}\right)=g_{2}} \bigwedge_{m_{2} \in M_{2}}\left(\varphi \circ t \circ \beta^{o p}\left(m_{2}\right)\right. \\
& \left.\longrightarrow{ }_{r} I_{2}\left(\alpha\left(g_{1}\right), m_{2}\right)\right) \\
& =\bigvee_{\alpha\left(g_{1}\right)=g_{2}, m_{2} \in M_{2}}\left(\varphi \circ t \circ \beta^{o p}\left(m_{2}\right) \longrightarrow_{r} I_{2}\left(g_{2}, m_{2}\right)\right) \\
& \stackrel{(\dagger)}{=} \bigwedge_{m_{2} \in M_{2}}\left(\varphi \circ t \circ \beta^{o p}\left(m_{2}\right) \longrightarrow_{r} I_{2}\left(g_{2}, m_{2}\right)\right) \\
& =\left(K_{2}\left(\varphi \circ t \circ \beta^{o p}\right)\right)\left(g_{2}\right)=\left(K_{2} \circ(\beta, \varphi)^{\leftarrow o}(t)\right)\left(g_{2}\right) \text {, }
\end{aligned}
$$

where $(\dagger),(\dagger \dagger)$ use the definition of the category $\mathbf{S}-\mathbf{F C}_{m *}^{C}$, whereas ( $\dagger \dagger \dagger$ ) employs the fact that $\mathscr{K}_{1} \stackrel{f}{\rightarrow} \mathscr{K}_{2}$ is a morphism of the category $\mathbf{S}-\mathbf{F C}_{m}^{C}$.

In the notation " $\mathrm{H}_{\mathrm{CmDa}}$ ", " $\mathrm{Cm}$ " shows its domain, and " $D a$ " (short for " $D M R a$ ") stands for its codomain. Similar notational conventions will be used in the remainder of this paper but will not be mentioned explicitly again.

In the following, we make the new functor into an embedding. The result depends on a particular subcategory of its domain.

Definition 49. Suppose that the variety $\mathbf{L}$, of which $\mathbf{S}$ is a subcategory, extends the variety UQuant of unital quantales. $\mathbf{S}-\mathbf{F C}_{m * *}^{C}$ (resp., $\mathbf{S}-\mathbf{F C}_{m *}^{C}$ ) is the full subcategory of the category S-FC $\mathbf{C}_{m *}^{C}$, whose objects $\mathscr{K}=(G, M, L, I)$ are such that $G$ is nonempty (resp., $M$ is nonempty) and, moreover, $1_{L} \neq \perp_{L}$.

The notation $(-)_{* *}$ (resp., $(-)_{* *}$ ) will be used for many of the subcategories constructed in this paper, the scope of each being limited to its respective subsection. Additionally, more important constructions will be distinguished in a different way. It is also important to emphasize that the algebra requirement of Definition 49 is satisfied by the crisp case (see Definition 31(1)).

Theorem 50. The restriction $H_{C m D a}^{*}\left(\right.$ resp., $\left.H_{C m D a}^{*}\right)$ of the functor $\mathbf{S}-\mathbf{F C}_{m *}^{C} \stackrel{\mathrm{H}_{\mathrm{CmDa} a}}{\longrightarrow} \mathbf{S}-\mathbf{F C}_{a}^{D M R}$ to $\mathbf{S}-\mathbf{F C}_{m * *}^{C}$ (resp., $\mathbf{S}-\mathbf{F C}_{m * \bullet}^{\mathrm{C}}$ ) gives an (in general, nonfull) embedding of the latter category into $\mathbf{S}-\mathbf{F C}_{a}^{D M R}$.

Proof. We consider the case of the functor $H_{C m D a}^{*}$ first and show its faithfulness. Given $\mathscr{K}_{1} \underset{f_{2}}{\stackrel{f_{1}}{\rightrightarrows}} \mathscr{K}_{2}$ in $\mathbf{S}-\mathbf{F C}_{m * *}^{C}$ with $H_{C m D a}^{*}\left(f_{1}\right)=H_{C m D a}^{*}\left(f_{2}\right)$, it follows that $\left(\alpha_{1}, \varphi_{1}\right) \rightarrow=$ $\left(\alpha_{2}, \varphi_{2}\right) \rightarrow,\left(\beta_{1}, \varphi_{1}\right)^{\leftarrow o}=\left(\beta_{2}, \varphi_{2}\right)^{\leftarrow o}$.

To show that $\alpha_{1}=\alpha_{2}$ (which we will refer to as $\alpha$ ), notice that given $g_{1} \in G_{1}$,

$$
\begin{aligned}
\left(\left(\alpha_{1}, \varphi_{1}\right) \rightarrow\left(\chi_{\left\{g_{1}\right\}}^{1_{L_{1}}}\right)\right)\left(g_{2}\right) & =\varphi_{1}\left(\bigvee_{\alpha_{1}\left(g_{1}^{\prime}\right)=g_{2}} \chi_{\left\{g_{1}\right\}}^{1_{L_{1}}}\left(g_{1}^{\prime}\right)\right) \\
& =\varphi_{1}\left(\left\{\begin{array}{ll}
1_{L_{1}}, & g_{2}=\alpha_{1}\left(g_{1}\right) \\
\perp_{L_{1}}, & \text { otherwise }
\end{array}\right)\right. \\
& = \begin{cases}1_{L_{2}}, & g_{2}=\alpha_{1}\left(g_{1}\right) \\
\perp_{L_{2}}, & \text { otherwise }\end{cases} \\
& =\chi_{\left\{\alpha_{1}\left(g_{1}\right)\right\}}^{1_{L_{2}}}\left(g_{2}\right),
\end{aligned}
$$

for every $g_{2} \in G_{2}$. As a consequence, we obtain that $\chi_{\left\{\alpha_{1}\left(g_{1}\right)\right\}}^{1_{L_{2}}}=$ $\left(\alpha_{1}, \varphi_{1}\right) \rightarrow\left(\chi_{\left\{g_{1}\right\}}^{1_{L_{1}}}\right)=\left(\alpha_{2}, \varphi_{2}\right) \rightarrow\left(\chi_{\left\{g_{1}\right\}}^{1_{L_{1}}}\right)=\chi_{\left\{\alpha_{2}\left(g_{1}\right)\right\}}^{1_{L_{2}}}$ and, therefore, $\alpha_{1}\left(g_{1}\right)=\alpha_{2}\left(g_{1}\right)$, by our assumption that $1_{L_{2}} \neq \perp_{L_{2}}$.

To show that $\beta_{1}^{o p}=\beta_{2}^{o p}=\beta^{o p}$, notice that given $m_{2} \in M_{2}$, $\left(\left(\beta_{1}, \varphi_{1}\right)^{\leftarrow o}\left(\chi_{\left\{\beta_{1}^{o p}\left(m_{2}\right)\right\}}^{1_{L_{1}}}\right)\right)\left(m_{2}\right)=\varphi_{1} \circ \chi_{\left\{\beta_{1}^{o p}\left(m_{2}\right)\right\}}^{1_{L_{1}}} \circ \beta_{1}^{o p}\left(m_{2}\right)=1_{L_{2}}$. Additionally,

$$
\begin{gathered}
\left(\left(\beta_{2}, \varphi_{2}\right)^{\leftarrow o}\left(\chi_{\left\{\beta_{1}^{o p}\left(m_{2}\right)\right\}}^{1_{L_{1}}}\right)\right)\left(m_{2}\right) \\
=\varphi_{2} \circ \chi_{\left\{\beta_{1}^{o p}\left(m_{2}\right)\right\}}^{1_{L_{1}}} \circ \beta_{2}^{o p}\left(m_{2}\right) \\
= \begin{cases}1_{L_{2}}, & \beta_{2}^{o p}\left(m_{2}\right)=\beta_{1}^{o p}\left(m_{2}\right) \\
\perp_{L_{2}}, & \text { otherwise. }\end{cases}
\end{gathered}
$$

Since $\quad\left(\left(\beta_{1}, \varphi_{1}\right)^{\leftarrow o}\left(\chi_{\left\{\beta_{1}^{o p}\left(m_{2}\right)\right\}}^{1_{L_{1}}}\right)\right)\left(m_{2}\right)$

$\left(\left(\beta_{2}, \varphi_{2}\right)^{\leftarrow o}\left(\chi_{\left\{\beta_{1}^{\text {op }}\left(m_{2}\right)\right\}}^{1_{L_{1}}}\right)\right)\left(m_{2}\right)$, it immediately follows that $\beta_{1}^{o p}\left(m_{2}\right)=\beta_{2}^{o p}\left(m_{2}\right)$, by our assumption that $1_{L_{2}} \neq \perp_{L_{2}}$. 
To show that $\varphi_{1}=\varphi_{2}=\varphi$, notice that given $a_{1} \in L_{1}$, we can choose some $g_{1} \in G_{1}\left(G_{1}\right.$ is nonempty). Then $\left(\left(\alpha, \varphi_{1}\right) \rightarrow\left(\underline{a_{1}}\right)\right)\left(\alpha\left(g_{1}\right)\right)=\varphi_{1}\left(\bigvee_{\alpha\left(g_{1}^{\prime}\right)=\alpha\left(g_{1}\right)} a_{1}\left(g_{1}^{\prime}\right)\right)=$ $\varphi_{1}\left(a_{1}\right)$ implies $\varphi_{1}\left(a_{1}\right)=\left(\left(\alpha, \varphi_{1}\right) \rightarrow\left(a_{1}\right)\right)\left(\alpha\left(g_{1}\right)\right)=$ $\left(\left(\alpha, \varphi_{2}\right) \rightarrow\left(a_{1}\right)\right)\left(\alpha\left(g_{1}\right)\right)=\varphi_{2}\left(a_{1}\right)$.

Turning the attention to the functor $H_{\mathrm{CmDa}}^{\circ}$, one follows the same steps to obtain $\alpha_{1}=\alpha_{2}=\alpha$ and $\beta_{1}^{o p}=\beta_{2}^{o p}=\beta^{o p}$. The only difference is in the proof of $\varphi_{1}=\varphi_{2}=\varphi$. Given $a_{1} \in L_{1}$, we choose some $m_{2} \in M_{2}$ ( $M_{2}$ is nonempty). It follows then that $\left(\left(\beta, \varphi_{1}\right)^{\leftarrow o}\left(a_{1}\right)\right)\left(m_{2}\right)=\varphi_{1} \circ a_{1} \circ \beta^{\circ p}\left(m_{2}\right)=$ $\varphi_{1}\left(a_{1}\right)$ and, therefore, $\varphi_{1}\left(a_{1}\right)=\left(\left(\beta, \varphi_{1}\right)^{\leftarrow o}\left(a_{1}\right)\right)\left(m_{2}\right)=$ $\left(\left(\beta, \varphi_{2}\right)^{\leftarrow o}\left(a_{1}\right)\right)\left(m_{2}\right)=\varphi_{2}\left(a_{1}\right)$.

For the nonfullness claim, we restrict the setting to the crisp case $\left(\mathbf{L}=\right.$ CBAlg and $\left.\mathbf{S}=\mathbf{S}_{2}\right)$ and consider the context $\mathscr{K}=(G, M, I)$, where $G=M=|2|$ and $I=$ $G \times M$. By Lemma 43 , it follows that $\mathscr{P}(G) \stackrel{H=\underline{M}}{\longrightarrow} \mathscr{P}(M)$ and $\mathscr{P}(M) \stackrel{K=\underline{G}}{\longrightarrow} \mathscr{P}(G)$. We define a formal context morphism $\mathscr{K} \stackrel{f}{\rightarrow} \mathscr{K}$ of the category $2-\mathrm{FC}_{a}^{D M R}$ by $\mathscr{P}(G) \stackrel{\alpha=\underline{G}}{\longrightarrow} \mathscr{P}(G)$ and $\mathscr{P}(M) \stackrel{\beta=\underline{M}}{\longrightarrow} \mathscr{P}(M)$. Since the setting fits both the functor $H_{\mathrm{CmDa}}^{*}$ and $H_{\mathrm{C} m \mathrm{Da}}^{\circ}$, we consider the case of the former one. Suppose there exists some $\mathscr{K} \stackrel{f^{\prime}}{\longrightarrow} \mathscr{K}$ in $2-\mathbf{F C}_{m * *}^{C}$ such that $H_{C m D a}^{*}\left(f^{\prime}\right)=f$. If follows that $\alpha=\left(\alpha^{\prime}\right) \rightarrow$ (crisp forward powerset operator) and, therefore, $\left\{\alpha^{\prime}(\perp)\right\}=\left(\alpha^{\prime}\right) \rightarrow(\{\perp\})=$ $\alpha(\{\perp\})=G$, which is an obvious contradiction.

5.1.2. From $\mathbf{S}-\mathbf{F C}_{m}^{C}$ to $\mathbf{S}-\mathbf{F C}^{D M R}$. In this subsection, we construct a functorial embedding of a particular subcategory of the category $\mathbf{S}-\mathbf{F} \mathbf{C}_{m}^{C}$ into the category $\mathbf{S}-\mathbf{F C} \mathbf{C}^{D M R}$. We begin again with singling out the subcategory in question.

Definition 51. S-FC ${ }_{m *}^{C}$ is the (nonfull) subcategory of the category $\mathbf{S}-\mathbf{F C}_{m}^{C}$, with the same objects, whose morphisms $\mathscr{K}_{1} \stackrel{f}{\rightarrow} \mathscr{K}_{2}$ are such that the maps $G_{1} \stackrel{\alpha}{\rightarrow} G_{2}, M_{2} \stackrel{\beta^{o p}}{\longrightarrow}$ $M_{1}$ are surjective, whereas the $\mathbf{S}$-morphism $L_{1} \stackrel{\varphi}{\rightarrow} L_{2}$ is an isomorphism.

With the new definition in hand, we can construct the following functor.

Theorem 52. There exists the functor $\mathbf{S}-\mathrm{FC}_{m *}^{C} \stackrel{H_{\mathrm{C} m D}}{\longrightarrow}$ S-FC ${ }^{D M R}$, which is given by $H_{C m D}\left(\mathscr{K}_{1} \stackrel{f}{\rightarrow} \mathscr{K}_{2}\right)=$ $\mathscr{K}_{1} \stackrel{\left((\alpha, \varphi)^{\rightarrow},\left((\beta, \varphi)^{\vdash \circ o}\right)^{o p}\right)}{\longrightarrow} \mathscr{K}_{2}$.

Proof. To show that the functor is correct on morphisms, we verify commutativity of the diagrams

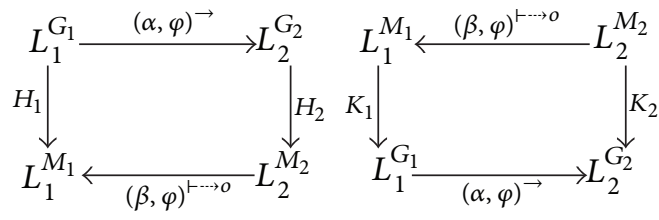

For the left-hand diagram, notice that given $s \in L_{1}^{G_{1}}$ and $m_{1} \in M_{1}$,

$$
\begin{aligned}
& \left((\beta, \varphi)^{\vdash \rightarrow o} \circ H_{2} \circ(\alpha, \varphi)^{\rightarrow}(s)\right)\left(m_{1}\right) \\
& =\varphi^{\vdash}\left(\bigvee_{\beta^{\circ P}\left(m_{2}\right)=m_{1}}\left(H_{2} \circ(\alpha, \varphi) \rightarrow(s)\right)\left(m_{2}\right)\right) \\
& =\varphi^{\vdash}\left(\bigvee _ { \beta ^ { o p } ( m _ { 2 } ) = m _ { 1 } } \left(\bigwedge _ { g _ { 2 } \in G _ { 2 } } \left(((\alpha, \varphi) \rightarrow(s))\left(g_{2}\right)\right.\right.\right. \\
& \left.\left.\left.\longrightarrow_{l} I_{2}\left(g_{2}, m_{2}\right)\right)\right)\right) \\
& =\varphi^{\vdash}\left(\bigvee _ { \beta ^ { \circ p } ( m _ { 2 } ) = m _ { 1 } } \left(\bigwedge _ { g _ { 2 } \in G _ { 2 } } \left(\varphi\left(\underset{\alpha\left(g_{1}\right)=g_{2}}{\bigvee} s\left(g_{1}\right)\right)\right.\right.\right. \\
& \left.\left.\left.\longrightarrow_{l} I_{2}\left(g_{2}, m_{2}\right)\right)\right)\right) \\
& =\varphi^{\vdash}\left(\bigvee _ { \beta ^ { o p } ( m _ { 2 } ) = m _ { 1 } } \left(\bigwedge _ { g _ { 2 } \in G _ { 2 } } \left(\left(\bigvee_{\alpha\left(g_{1}\right)=g_{2}} \varphi \circ s\left(g_{1}\right)\right)\right.\right.\right. \\
& \left.\left.\left.\longrightarrow_{l} I_{2}\left(g_{2}, m_{2}\right)\right)\right)\right) \\
& =\varphi^{\vdash}\left(\bigvee _ { \beta ^ { \circ p } ( m _ { 2 } ) = m _ { 1 } } \left(\bigwedge _ { g _ { 2 } \in G _ { 2 } } \left(\bigwedge _ { \alpha ( g _ { 1 } ) = g _ { 2 } } \left(\varphi \circ s\left(g_{1}\right)\right.\right.\right.\right. \\
& \left.\left.\left.\longrightarrow_{l} I_{2}\left(g_{2}, m_{2}\right)\right)\right)\right) \\
& =\varphi^{\vdash}\left(\bigvee _ { \beta ^ { \circ p } ( m _ { 2 } ) = m _ { 1 } } \left(\bigwedge _ { g _ { 1 } \in G _ { 1 } } \left(\varphi \circ s\left(g_{1}\right)\right.\right.\right. \\
& \left.\left.\left.\longrightarrow_{l} I_{2}\left(\alpha\left(g_{1}\right), m_{2}\right)\right)\right)\right) \\
& \stackrel{(\dagger)}{=} \varphi^{\vdash}\left(\bigvee _ { \beta ^ { \circ p } ( m _ { 2 } ) = m _ { 1 } } \left(\bigwedge _ { g _ { 1 } \in G _ { 1 } } \left(\varphi \circ s\left(g_{1}\right)\right.\right.\right. \\
& \left.\left.\left.\longrightarrow_{l} \varphi \circ I_{1}\left(g_{1}, \beta^{o p}\left(m_{2}\right)\right)\right)\right)\right)
\end{aligned}
$$




$$
\begin{aligned}
& =\varphi^{\vdash}\left(\bigvee _ { \beta ^ { \circ p } ( m _ { 2 } ) = m _ { 1 } } \left(\bigwedge _ { g _ { 1 } \in G _ { 1 } } \left(\varphi \circ s\left(g_{1}\right)\right.\right.\right. \\
& \left.\left.\left.\longrightarrow_{l} \varphi \circ I_{1}\left(g_{1}, m_{1}\right)\right)\right)\right) \\
& \stackrel{(\dagger+)}{=} \varphi^{\vdash}\left(\bigwedge_{g_{1} \in G_{1}}\left(\varphi \circ s\left(g_{1}\right) \longrightarrow_{l} \varphi \circ I_{1}\left(g_{1}, m_{1}\right)\right)\right) \\
& \stackrel{(\dagger+)}{=} \varphi^{\vdash} \circ \varphi\left(\bigwedge_{g_{1} \in G_{1}}\left(s\left(g_{1}\right) \longrightarrow{ }_{l} I_{1}\left(g_{1}, m_{1}\right)\right)\right) \\
& \stackrel{(\dagger+)}{=} \bigwedge_{g_{1} \in G_{1}}\left(s\left(g_{1}\right) \longrightarrow I_{1}\left(g_{1}, m_{1}\right)\right)=\left(H_{1}(s)\right)\left(m_{1}\right) \text {, }
\end{aligned}
$$

where $(\dagger)$ relies on the fact that $\mathscr{K}_{1} \stackrel{f}{\rightarrow} \mathscr{K}_{2}$ is a morphism of the category $\mathbf{S}-\mathbf{F} \mathbf{C}_{m}^{C}$, whereas $(\dagger \dagger)$ employs the definition of the category $\mathbf{S}-\mathbf{F C}_{m *}^{C}$ (notice that the inverse of $\varphi$ is easily seen to be $\varphi^{\vdash}$ ).

For the right-hand diagram, notice that given $t \in L_{2}^{M_{2}}$ and $g_{2} \in G_{2}$,

$$
\begin{aligned}
& \left((\alpha, \varphi) \rightarrow \circ K_{1} \circ(\beta, \varphi)^{\vdash \rightarrow o}(t)\right)\left(g_{2}\right) \\
& =\varphi\left(\bigvee_{\alpha\left(g_{1}\right)=g_{2}}\left(K_{1} \circ(\beta, \varphi)^{\vdash \rightarrow o}(t)\right)\left(g_{1}\right)\right) \\
& =\varphi\left(\bigvee _ { \alpha ( g _ { 1 } ) = g _ { 2 } } \left(\bigwedge _ { m _ { 1 } \in M _ { 1 } } \left(\left((\beta, \varphi)^{\vdash-\infty o o}(t)\right)\left(m_{1}\right)\right.\right.\right. \\
& \left.\left.\left.\longrightarrow_{r} I_{1}\left(g_{1}, m_{1}\right)\right)\right)\right) \\
& =\varphi\left(\bigvee _ { \alpha ( g _ { 1 } ) = g _ { 2 } } \left(\bigwedge _ { m _ { 1 } \in M _ { 1 } } \left(\varphi^{\vdash}\left(\bigvee_{\beta^{o p}\left(m_{2}\right)=m_{1}} t\left(m_{2}\right)\right)\right.\right.\right. \\
& \left.\left.\left.\longrightarrow_{r} I_{1}\left(g_{1}, m_{1}\right)\right)\right)\right) \\
& =\varphi\left(\underset { \alpha ( g _ { 1 } ) = g _ { 2 } } { \bigvee } \left(\bigwedge _ { m _ { 1 } \in M _ { 1 } } \left(\left(\bigvee_{\beta^{\circ p}\left(m_{2}\right)=m_{1}} \varphi^{\vdash} \circ t\left(m_{2}\right)\right)\right.\right.\right. \\
& \left.\left.\left.\longrightarrow_{r} I_{1}\left(g_{1}, m_{1}\right)\right)\right)\right)
\end{aligned}
$$

$$
\begin{aligned}
& =\varphi\left(\bigvee _ { \alpha ( g _ { 1 } ) = g _ { 2 } } \left(\bigwedge _ { m _ { 1 } \in M _ { 1 } } \left(\bigwedge _ { \beta ^ { \circ p } ( m _ { 2 } ) = m _ { 1 } } \left(\varphi^{\vdash} \circ t\left(m_{2}\right)\right.\right.\right.\right. \\
& \left.\left.\left.\longrightarrow_{r} I_{1}\left(g_{1}, m_{1}\right)\right)\right)\right) \\
& =\varphi\left(\bigvee _ { \alpha ( g _ { 1 } ) = g _ { 2 } } \left(\bigwedge _ { m _ { 2 } \in M _ { 2 } } \left(\varphi^{\vdash} \circ t\left(m_{2}\right)\right.\right.\right. \\
& \left.\left.\left.\longrightarrow_{r} I_{1}\left(g_{1}, \beta^{o p}\left(m_{2}\right)\right)\right)\right)\right) \\
& \stackrel{(\dagger)}{=} \bigvee_{\alpha\left(g_{1}\right)=g_{2}} \bigwedge_{2_{2} \in M_{2}}\left(\varphi \circ \varphi^{\vdash} \circ t\left(m_{2}\right)\right. \\
& \left.\longrightarrow_{r} \varphi \circ I_{1}\left(g_{1}, \beta^{o p}\left(m_{2}\right)\right)\right) \\
& \stackrel{(\dagger \dagger)}{=} \bigvee_{\alpha\left(g_{1}\right)=g_{2}} \bigwedge_{m_{2} \in M_{2}}\left(\varphi \circ \varphi^{\vdash} \circ t\left(m_{2}\right) \longrightarrow_{r} I_{2}\left(\alpha\left(g_{1}\right), m_{2}\right)\right) \\
& =\bigvee_{\alpha\left(g_{1}\right)=g_{2}} \bigwedge_{m_{2} \in M_{2}}\left(\varphi \circ \varphi^{\vdash} \circ t\left(m_{2}\right) \longrightarrow_{r} I_{2}\left(g_{2}, m_{2}\right)\right) \\
& \stackrel{(\dagger)}{=} \bigwedge_{m_{2} \in M_{2}}\left(\varphi \circ \varphi^{\vdash} \circ t\left(m_{2}\right) \longrightarrow_{r} I_{2}\left(g_{2}, m_{2}\right)\right) \\
& \stackrel{(\dagger)}{=} \bigwedge_{m_{2} \in M_{2}}\left(t\left(m_{2}\right) \longrightarrow{ }_{r} I_{2}\left(g_{2}, m_{2}\right)\right)=\left(K_{2}(t)\right)\left(g_{2}\right) \text {, }
\end{aligned}
$$

where ( $\dagger$ ) uses the definition of the category $\mathbf{S}-\mathbf{F C}_{m *}^{C}$, whereas $(\dagger \dagger)$ relies on the fact that $\mathscr{K}_{1} \stackrel{f}{\rightarrow} \mathscr{K}_{2}$ is a morphism of the category $\mathbf{S}-\mathbf{F C}_{m}^{C}$.

The category S-FC $\mathbf{C}_{m * *}^{C}$ (resp., S-FC $\mathbf{C}_{m *}^{C}$ ) of Definition 49 provides the following result.

Theorem 53. The restriction $H_{C m D}^{*}$ (resp., $H_{C m D}^{*}$ ) of the functor $\mathbf{S}-\mathbf{F C}_{m *}^{C} \stackrel{H_{\mathrm{CmD}}}{\longrightarrow} \mathbf{S}-\mathbf{F C}^{D M R}$ to $\mathbf{S}-\mathbf{F C}_{m * *}^{\mathrm{C}}$ (resp., S-FC ${ }_{m * *}^{C}$ ) gives an (in general, nonfull) embedding of the latter category into $\mathbf{S}-\mathbf{F C}^{D M R}$.

Proof. Follow the steps of the proof of Theorem 50, where the backward powerset operator $(\beta, \varphi)^{\leftarrow o}$ is substituted by the forward powerset operator $(\beta, \varphi)^{\vdash-\rightarrow o}$.

5.1.3. From $\mathbf{S}-\mathbf{F C} C^{C}$ to $\mathbf{S}-\mathbf{F C}_{a}^{D M R}$. In this subsection, we construct a functorial embedding of a particular subcategory of the category $\mathbf{S}-\mathbf{F C}{ }^{C}$ into the category $\mathbf{S}-\mathbf{F C}_{a}^{D M R}$. We start by defining the subcategory in question.

Definition 54. S- $\mathbf{F C}_{*}^{C}$ is the (nonfull) subcategory of the category $\mathbf{S}-\mathbf{F C}^{C}$, with the same objects, whose morphisms 
$\mathscr{K}_{1} \stackrel{f}{\rightarrow} \mathscr{K}_{2}$ are such that the maps $G_{1} \stackrel{\alpha}{\rightarrow} G_{2}, M_{2} \stackrel{\beta^{o p}}{\longrightarrow} M_{1}$ are surjective, whereas the $S^{o p}$-morphism $L_{2} \stackrel{\varphi^{o p}}{\longrightarrow} L_{1}$ is injective, and, moreover, $\left|L_{1}\right| \stackrel{\varphi^{o p \vdash}}{\longrightarrow}\left|L_{2}\right|$ preserves $\bigvee, \rightarrow_{l}$ and $\rightarrow_{r}$.

For the new category in hand, in the following, we construct its respective functor.

Theorem 55. There exists the functor $\mathbf{S}-\mathbf{F C}_{*}^{C} \stackrel{H_{\mathrm{CDa}}}{\longrightarrow} \mathbf{S}-\mathbf{F C}_{a}^{D M R}$, which is given by $H_{\mathrm{CDa}}\left(\mathscr{K}_{1} \stackrel{f}{\rightarrow} \mathscr{K}_{2}\right)=\mathscr{K}_{1} \stackrel{\left((\alpha, \varphi)^{\vdash \cdots},\left((\beta, \varphi)^{\vdash \cdots 0}\right)^{o p}\right)}{\longrightarrow}$ $\mathscr{K}_{2}$.

Proof. To show that the functor is correct on morphisms, we verify commutativity of the diagrams

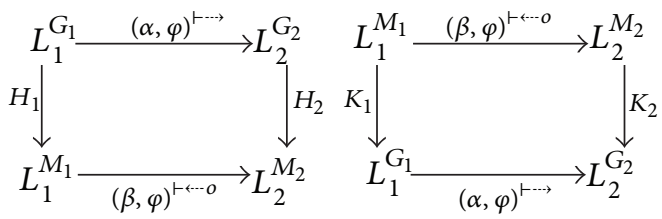

For the left-hand diagram, notice that given $s \in L_{1}^{G_{1}}$ and $m_{2} \in M_{2}$,

$$
\begin{aligned}
& \left(H_{2} \circ(\alpha, \varphi)^{\vdash \rightarrow(}(s)\right)\left(m_{2}\right) \\
& =\bigwedge_{g_{2} \in G_{2}}\left(\left((\alpha, \varphi)^{\vdash \cdots(}(s)\right)\left(g_{2}\right) \longrightarrow_{l} I_{2}\left(g_{2}, m_{2}\right)\right) \\
& =\bigwedge_{g_{2} \in G_{2}}\left(\varphi^{o p \vdash}\left(\underset{\alpha\left(g_{1}\right)=g_{2}}{\bigvee} s\left(g_{1}\right)\right) \rightarrow_{l} I_{2}\left(g_{2}, m_{2}\right)\right) \\
& \stackrel{(†)}{=} \bigwedge_{g_{2} \in G_{2}}\left(\left(\bigvee_{\alpha\left(g_{1}\right)=g_{2}} \varphi^{o p \vdash} \circ s\left(g_{1}\right)\right) \longrightarrow I_{l} I_{2}\left(g_{2}, m_{2}\right)\right) \\
& =\bigwedge_{g_{2} \in G_{2}} \bigwedge_{\alpha\left(g_{1}\right)=g_{2}}\left(\varphi^{o p \vdash} \circ s\left(g_{1}\right) \longrightarrow{ }_{l} I_{2}\left(g_{2}, m_{2}\right)\right) \\
& =\bigwedge_{g_{1} \in G_{1}}\left(\varphi^{o p \vdash} \circ s\left(g_{1}\right) \longrightarrow{ }_{l} I_{2}\left(\alpha\left(g_{1}\right), m_{2}\right)\right) \\
& \stackrel{(†)}{=} \bigwedge_{g_{1} \in G_{1}}\left(\varphi^{o p \vdash} \circ s\left(g_{1}\right) \longrightarrow l \varphi^{o p \vdash} \circ \varphi^{o p} \circ I_{2}\left(\alpha\left(g_{1}\right), m_{2}\right)\right) \\
& \stackrel{(\dagger)}{=} \varphi^{o p}\left(\bigwedge_{g_{1} \in G_{1}}\left(s\left(g_{1}\right) \longrightarrow_{l} \varphi^{o p} \circ I_{2}\left(\alpha\left(g_{1}\right), m_{2}\right)\right)\right) \\
& \stackrel{(\dagger \dagger)}{=} \varphi^{o p \vdash}\left(\bigwedge_{g_{1} \in G_{1}}\left(s\left(g_{1}\right) \longrightarrow_{l} I_{1}\left(g_{1}, \beta^{o p}\left(m_{2}\right)\right)\right)\right) \\
& =\left(\varphi^{o p \vdash} \circ H_{1}(s) \circ \beta^{o p}\right)\left(m_{2}\right) \\
& =\left((\beta, \varphi)^{1 \cdots o} \circ H_{1}(s)\right)\left(m_{2}\right) \text {, }
\end{aligned}
$$

where $(\dagger)$ uses Definition 54 (notice that $\varphi^{o p \vdash}$ is a left inverse to $\varphi^{o p}$ ), whereas $(\dagger \dagger)$ relies on the fact that $\mathscr{K}_{1} \stackrel{f}{\rightarrow} \mathscr{K}_{2}$ is a morphism of $\mathbf{S}-\mathbf{F C}^{\mathrm{C}}$.

For the right-hand diagram, notice that given $t \in L_{1}^{M_{1}}$ and $g_{2} \in G_{2}$,

$$
\begin{aligned}
& \left((\alpha, \varphi)^{\vdash \cdots \rightarrow} \circ K_{1}(t)\right)\left(g_{2}\right) \\
& =\varphi^{o p \vdash}\left(\bigvee_{\alpha\left(g_{1}\right)=g_{2}}\left(K_{1}(t)\right)\left(g_{1}\right)\right) \\
& \quad=\varphi^{o p \vdash}\left(\bigvee_{\alpha\left(g_{1}\right)=g_{2}}\left(\bigwedge_{m_{1} \in M_{1}}\left(t\left(m_{1}\right) \longrightarrow_{r} I_{1}\left(g_{1}, m_{1}\right)\right)\right)\right) \\
& \stackrel{(+)}{=} \varphi^{o p \vdash}\left(\bigvee _ { \alpha ( g _ { 1 } ) = g _ { 2 } } \left(\bigwedge _ { m _ { 2 } \in M _ { 2 } } \left(t \circ \beta^{o p}\left(m_{2}\right)\right.\right.\right. \\
& \left.\left.\left.\longrightarrow I_{1} I_{1}\left(g_{1}, \beta^{o p}\left(m_{2}\right)\right)\right)\right)\right)
\end{aligned}
$$

$$
\stackrel{(\dagger \dagger)}{=} \varphi^{o p \vdash}\left(\bigvee _ { \alpha ( g _ { 1 } ) = g _ { 2 } } \left(\bigwedge _ { m _ { 2 } \in M _ { 2 } } \left(t \circ \beta^{o p}\left(m_{2}\right)\right.\right.\right.
$$$$
\left.\left.\left.\longrightarrow_{r} \varphi^{o p} \circ I_{2}\left(\alpha\left(g_{1}\right), m_{2}\right)\right)\right)\right)
$$

$$
\begin{aligned}
=\varphi^{o p \vdash}\left(\bigvee _ { \alpha ( g _ { 1 } ) = g _ { 2 } } \left(\bigwedge_{m_{2} \in M_{2}}\right.\right. & \left(t \circ \beta^{o p}\left(m_{2}\right)\right. \\
& \left.\left.\left.\longrightarrow r \varphi^{o p} \circ I_{2}\left(g_{2}, m_{2}\right)\right)\right)\right)
\end{aligned}
$$

$\stackrel{(\dagger)}{=} \varphi^{o p \vdash}\left(\bigwedge_{m_{2} \in M_{2}}\left(t \circ \beta^{o p}\left(m_{2}\right) \longrightarrow_{r} \varphi^{o p} \circ I_{2}\left(g_{2}, m_{2}\right)\right)\right)$

$\stackrel{(\dagger)}{=} \bigwedge_{m_{2} \in M_{2}}\left(\varphi^{o p \vdash} \circ t \circ \beta^{o p}\left(m_{2}\right) \longrightarrow_{r} \varphi^{o p \vdash} \circ \varphi^{o p} \circ I_{2}\left(g_{2}, m_{2}\right)\right)$

$\stackrel{(\dagger)}{=} \bigwedge_{m_{2} \in M_{2}}\left(\varphi^{o p \vdash} \circ t \circ \beta^{o p}\left(m_{2}\right) \longrightarrow_{r} I_{2}\left(g_{2}, m_{2}\right)\right)$

$$
\begin{aligned}
& =\left(K_{2}\left(\varphi^{o p \vdash} \circ t \circ \beta^{o p}\right)\right)\left(g_{2}\right) \\
& =\left(K_{2} \circ(\beta, \varphi)^{\vdash \cdots o}(t)\right)\left(g_{2}\right),
\end{aligned}
$$

where $(\dagger)$ uses Definition 54, whereas $(\dagger \dagger)$ relies on the fact that $\mathscr{K}_{1} \stackrel{f}{\rightarrow} \mathscr{K}_{2}$ is a morphism of $\mathbf{S}-\mathbf{F C}^{\mathrm{C}}$. 
In the following, we make the new functor into an embedding.

Definition 56. Suppose that the variety $\mathbf{L}$, of which $\mathbf{S}$ is a subcategory, extends $\mathbf{U Q u a n t} \mathbf{S}-\mathbf{F C}_{* *}^{C}$ (resp., $\mathbf{S}-\mathbf{F C}_{*}^{C}$ ) is the (nonfull) subcategory of the category $\mathbf{S}-\mathbf{F C}_{*}^{C}$, comprising the following data.

Objects. S-FC $\mathbf{C}_{*}^{\mathrm{C}}$-objects $\mathscr{K}$, for which $G$ is nonempty (resp., $M$ is nonempty) and, moreover, $1_{L} \neq \perp_{L}$.

Morphisms. S-FC ${ }_{*}^{C}$-morphisms $\mathscr{K}_{1} \stackrel{f}{\rightarrow} \mathscr{K}_{2}$, for which $\left|L_{1}\right| \stackrel{\varphi^{o p \vdash}}{\longrightarrow}\left|L_{2}\right|$ is 1-preserving.

The reader should notice the essential difference in the setting of Definition 49, namely, an additional condition on the morphisms of the new category.

Theorem 57. The restriction $H_{C D a}^{*}$ (resp., $H_{C D a}^{*}$ ) of the functor $\mathbf{S}-\mathbf{F C}_{*}^{C} \stackrel{H_{C D a}}{\longrightarrow} \mathbf{S}-\mathbf{F C}_{a}^{D M R}$ to the category $\mathbf{S}-\mathbf{F C}_{* *}^{C}$ (resp., $\mathbf{S}-\mathbf{F C}_{*}^{\mathrm{C}}$ ) gives an (in general nonfull) embedding of the latter category into $\mathbf{S}-\mathrm{FC}_{a}^{D M R}$.

Proof. Follow the proof of Theorem 50, with the respective changes in the powerset operators.

5.1.4. From $\mathbf{S}-F \mathbf{C}^{C}$ to $\mathbf{S}-\mathbf{F C}^{D M R}$. In this subsection, we construct a functorial embedding of a particular subcategory of the category S-FC ${ }^{C}$ into the category S-FC ${ }^{D M R}$. As before, we begin with singling out the subcategory in question.

Definition 58. S-FC ${ }_{*}^{C}$ is the (nonfull) subcategory of the category $\mathbf{S}-\mathbf{F C}^{C}$, with the same objects, and whose morphisms $\mathscr{K}_{1} \stackrel{f}{\rightarrow} \mathscr{K}_{2}$ are such that the maps $G_{1} \stackrel{\alpha}{\rightarrow} G_{2}, M_{2} \stackrel{\beta^{o p}}{\longrightarrow} M_{1}$ are surjective, whereas the $\boldsymbol{S}^{o p}$-morphism $L_{2} \stackrel{\varphi^{o p}}{\longrightarrow} L_{1}$ is an isomorphism.

With the new category in hand, we are ready to define a new functor as follows.

Theorem 59. There exists the functor $\mathbf{S}-\mathbf{F C}_{*}^{C} \stackrel{H_{C D}}{\longrightarrow} \mathbf{S}-\mathbf{F C}^{D M R}$, which is given by $H_{C D}\left(\mathscr{K}_{1} \stackrel{f}{\rightarrow} \mathscr{K}_{2}\right)=\mathscr{K}_{1} \stackrel{\left((\alpha, \varphi)^{\vdash \cdots},\left((\beta, \varphi)^{\rightarrow 0}\right)^{o p}\right)}{\longrightarrow}$ $\mathscr{K}_{2}$.

Proof. To show that the functor is correct on morphisms, we verify commutativity of the diagrams

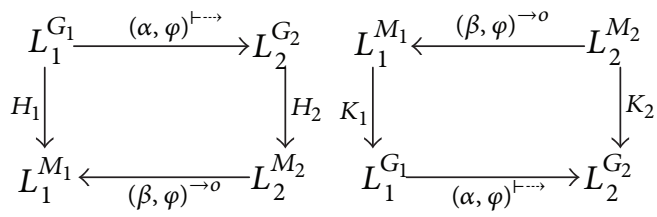

For the left-hand diagram, notice that given $s \in L_{1}^{G_{1}}$ and $m_{1} \in M_{1}$,

$$
\begin{aligned}
& \left((\beta, \varphi)^{\rightarrow o} \circ H_{2} \circ(\alpha, \varphi)^{\vdash \cdots \cdots}(s)\right)\left(m_{1}\right) \\
& =\varphi^{o p}\left(\bigvee_{\beta^{o p}\left(m_{2}\right)=m_{1}}\left(H_{2} \circ(\alpha, \varphi)^{\vdash-\rightarrow}(s)\right)\left(m_{2}\right)\right) \\
& =\varphi^{o p}\left(\bigvee _ { \beta ^ { o p } ( m _ { 2 } ) = m _ { 1 } } \left(\bigwedge _ { g _ { 2 } \in G _ { 2 } } \left(\left((\alpha, \varphi)^{\vdash \cdots)}(s)\right)\left(g_{2}\right)\right.\right.\right. \\
& \left.\left.\longrightarrow_{l} I_{2}\left(g_{2}, m_{2}\right)\right)\right) \\
& =\varphi^{o p}\left(\bigvee _ { \beta ^ { o p } ( m _ { 2 } ) = m _ { 1 } } \left(\bigwedge _ { g _ { 2 } \in G _ { 2 } } \left(\varphi^{o p \vdash}\left(\bigvee_{\alpha\left(g_{1}\right)=g_{2}} s\left(g_{1}\right)\right)\right.\right.\right. \\
& \left.\left.\left.\longrightarrow_{l} I_{2}\left(g_{2}, m_{2}\right)\right)\right)\right) \\
& \stackrel{(\dagger)}{=} \varphi^{o p}\left(\bigvee _ { \beta ^ { o p } ( m _ { 2 } ) = m _ { 1 } } \left(\bigwedge _ { g _ { 2 } \in G _ { 2 } } \left(\left(\bigvee_{\alpha\left(g_{1}\right)=g_{2}} \varphi^{o p \vdash} \circ s\left(g_{1}\right)\right)\right.\right.\right. \\
& \left.\left.\longrightarrow_{l} I_{2}\left(g_{2}, m_{2}\right)\right)\right) \text { ) } \\
& =\varphi^{o p}\left(\bigvee _ { \beta ^ { o p } ( m _ { 2 } ) = m _ { 1 } } \left(\bigwedge _ { g _ { 2 } \in G _ { 2 } } \left(\bigwedge _ { \alpha ( g _ { 1 } ) = g _ { 2 } } \left(\varphi^{o p \vdash} \circ s\left(g_{1}\right)\right.\right.\right.\right. \\
& \left.\left.\left.\left.\longrightarrow_{l} I_{2}\left(g_{2}, m_{2}\right)\right)\right)\right)\right) \\
& =\varphi^{o p}\left(\bigvee _ { \beta ^ { o p } ( m _ { 2 } ) = m _ { 1 } } \left(\bigwedge _ { g _ { 1 } \in G _ { 1 } } \left(\varphi^{o p \vdash} \circ s\left(g_{1}\right)\right.\right.\right. \\
& \left.\left.\left.\longrightarrow_{l} I_{2}\left(\alpha\left(g_{1}\right), m_{2}\right)\right)\right)\right) \\
& \stackrel{(†)}{=} \bigvee_{\beta^{o p}\left(m_{2}\right)=m_{1}} \bigwedge_{g_{1} \in G_{1}}\left(\varphi^{o p} \circ \varphi^{o p \vdash} \circ s\left(g_{1}\right)\right. \\
& \left.\longrightarrow_{l} \varphi^{o p} \circ I_{2}\left(\alpha\left(g_{1}\right), m_{2}\right)\right)
\end{aligned}
$$

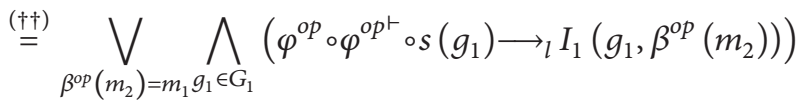

$$
\begin{aligned}
& \stackrel{(\dagger)}{=} \bigvee_{\beta^{o p}\left(m_{2}\right)=m_{1}} \bigwedge_{g_{1} \in G_{1}}\left(s\left(g_{1}\right) \longrightarrow_{l} I_{1}\left(g_{1}, \beta^{o p}\left(m_{2}\right)\right)\right)
\end{aligned}
$$




$$
\begin{aligned}
& =\bigvee_{\beta^{o p}\left(m_{2}\right)=m_{1}} \bigwedge_{g_{1} \in G_{1}}\left(s\left(g_{1}\right) \longrightarrow_{l} I_{1}\left(g_{1}, m_{1}\right)\right) \\
& \stackrel{(\dagger)}{=} \bigwedge_{g_{1} \in G_{1}}\left(s\left(g_{1}\right) \longrightarrow_{l} I_{1}\left(g_{1}, m_{1}\right)\right)=\left(H_{1}(s)\right)\left(m_{1}\right),
\end{aligned}
$$

where $(\dagger)$ uses Definition 58, whereas $(\dagger \dagger)$ relies on the fact that $\mathscr{K}_{1} \stackrel{f}{\rightarrow} \mathscr{K}_{2}$ is a morphism of $\mathbf{S}-\mathbf{F C}^{C}$.

For the right-hand diagram, notice that given $t \in L_{2}^{M_{2}}$ and $g_{2} \in G_{2}$,

$$
\begin{aligned}
& \left((\alpha, \varphi)^{\vdash \cdots \rightarrow} \circ K_{1} \circ(\beta, \varphi)^{\rightarrow o}(t)\right)\left(g_{2}\right) \\
& =\varphi^{o{ }^{\vdash}}\left(\bigvee_{\alpha\left(g_{1}\right)=g_{2}}\left(K_{1} \circ(\beta, \varphi)^{\rightarrow o}(t)\right)\left(g_{1}\right)\right) \\
& =\varphi^{o p^{\vdash}}\left(\bigvee _ { \alpha ( g _ { 1 } ) = g _ { 2 } } \left(\bigwedge _ { m _ { 1 } \in M _ { 1 } } \left(\left((\beta, \varphi)^{\rightarrow o}(t)\right)\left(m_{1}\right)\right.\right.\right. \\
& \left.\left.\longrightarrow_{r} I_{1}\left(g_{1}, m_{1}\right)\right)\right) \\
& =\varphi^{o p \vdash}\left(\bigvee _ { \alpha ( g _ { 1 } ) = g _ { 2 } } \left(\bigwedge _ { m _ { 1 } \in M _ { 1 } } \left(\varphi^{o p}\left(\bigvee_{\beta^{o p}\left(m_{2}\right)=m_{1}} t\left(m_{2}\right)\right)\right.\right.\right. \\
& \left.\left.\left.\longrightarrow_{r} I_{1}\left(g_{1}, m_{1}\right)\right)\right)\right) \\
& =\varphi^{o p \vdash}\left(\bigvee _ { \alpha ( g _ { 1 } ) = g _ { 2 } } \left(\bigwedge _ { m _ { 1 } \in M _ { 1 } } \left(\left(\bigvee_{\beta^{o p}\left(m_{2}\right)=m_{1}} \varphi^{o p} \circ t\left(m_{2}\right)\right)\right.\right.\right. \\
& \left.\left.\longrightarrow_{r} I_{1}\left(g_{1}, m_{1}\right)\right)\right) \\
& =\varphi^{o p \vdash}\left(\bigvee _ { \alpha ( g _ { 1 } ) = g _ { 2 } } \left(\bigwedge _ { m _ { 1 } \in M _ { 1 } } \left(\bigwedge _ { \beta ^ { o p } ( m _ { 2 } ) = m _ { 1 } } \left(\varphi^{o p} \circ t\left(m_{2}\right)\right.\right.\right.\right. \\
& \left.\left.\left.\longrightarrow_{r} I_{1}\left(g_{1}, m_{1}\right)\right)\right)\right) \\
& =\varphi^{o p}\left(\bigvee _ { \alpha ( g _ { 1 } ) = g _ { 2 } } \left(\bigwedge _ { m _ { 2 } \in M _ { 2 } } \left(\varphi^{o p} \circ t\left(m_{2}\right)\right.\right.\right. \\
& \left.\left.\left.\longrightarrow_{r} I_{1}\left(g_{1}, \beta^{o p}\left(m_{2}\right)\right)\right)\right)\right)
\end{aligned}
$$

$$
\begin{array}{r}
\stackrel{(\dagger)}{=} \varphi^{o p \vdash}\left(\bigvee _ { \alpha ( g _ { 1 } ) = g _ { 2 } } \left(\bigwedge _ { m _ { 2 } \in M _ { 2 } } \left(\varphi^{o p} \circ t\left(m_{2}\right)\right.\right.\right. \\
\\
\left.\left.\left.\longrightarrow r \varphi^{o p} \circ I_{2}\left(\alpha\left(g_{1}\right), m_{2}\right)\right)\right)\right)
\end{array}
$$$$
\stackrel{(\dagger \dagger)}{=} \varphi^{o p \vdash} \circ \varphi^{o p}\left(\bigvee _ { \alpha ( g _ { 1 } ) = g _ { 2 } } \left(\bigwedge _ { m _ { 2 } \in M _ { 2 } } \left(t\left(m_{2}\right)\right.\right.\right.
$$$$
\left.\left.\left.\longrightarrow{ }_{r} I_{2}\left(\alpha\left(g_{1}\right), m_{2}\right)\right)\right)\right)
$$$$
\stackrel{(\dagger \dagger)}{=} \bigvee_{\alpha\left(g_{1}\right)=g_{2}} \bigwedge_{m_{2} \in M_{2}}\left(t\left(m_{2}\right) \rightarrow_{r} I_{2}\left(\alpha\left(g_{1}\right), m_{2}\right)\right)
$$$$
=\bigvee_{\alpha\left(g_{1}\right)=g_{2}} \bigwedge_{m_{2} \in M_{2}}\left(t\left(m_{2}\right) \rightarrow_{r} I_{2}\left(g_{2}, m_{2}\right)\right)
$$$$
\stackrel{(\dagger \dagger)}{=} \bigwedge_{m_{2} \in M_{2}}\left(t\left(m_{2}\right) \longrightarrow I_{2}\left(g_{2}, m_{2}\right)\right)=\left(K_{2}(t)\right)\left(g_{2}\right) \text {, }
$$

where $(\dagger)$ uses the fact that $\mathscr{K}_{1} \stackrel{f}{\rightarrow} \mathscr{K}_{2}$ is a morphism of S-FC ${ }^{C}$, whereas $(\dagger \dagger)$ employs Definition 58.

Similar to Definition 49, one obtains the categories S-FC ${ }_{* *}^{C}$ and $\mathbf{S}-\mathbf{F C}_{* \bullet}^{C}$. Moreover, similar to Theorem 50, one gets the following result.

Theorem 60. The restriction $H_{C D}^{*}$ (resp., $H_{C D}^{\bullet}$ ) of the functor $\mathbf{S}-\mathbf{F C}_{*}^{C} \stackrel{H_{C D}}{\longrightarrow} \mathbf{S}-\mathbf{F C}^{D M R}$ to $\mathbf{S}-\mathbf{F C}_{* *}^{C}$ (resp., $\mathbf{S}-\mathbf{F C}_{* \bullet}^{C}$ ) gives an (in general nonfull) embedding of the latter category into S-FC ${ }^{D M R}$.

5.1.5. From $\mathbf{S}-\mathbf{F C}^{D M R}$ to $\mathbf{S}-\mathbf{F C} C^{C}$. In this subsection, we construct functorial embeddings of several subcategories of the category S-FC ${ }^{D M R}$ into the category $\mathbf{S}-\mathbf{F C}^{C}$ (most of them are motivated by [29]). We begin with defining the first subcategory in question. The reader should note that all the subcategories constructed in this subsection are fixed-basis.

Definition 61. Given an L-algebra $L, L-\mathbf{F C}_{\mathfrak{i}}^{D M R}$ is the (nonfull) subcategory of the category $L-F_{C} C^{D M R}$, with the same objects, whose morphisms $\mathscr{K}_{1} \stackrel{f}{\rightarrow} \mathscr{K}_{2}$ have the property that the maps $L^{G_{1}} \stackrel{\alpha}{\rightarrow} L^{G_{2}}, L^{M_{2}} \stackrel{\beta^{o p}}{\longrightarrow} L^{M_{1}}$ are injective.

The reader should notice that " $\mathfrak{i}$ " in $L-F_{\mathbf{i}}$ " "injective." 
Theorem 62. There exists the functor $L-\mathbf{F C}_{\mathfrak{i}}^{D M R} \stackrel{H_{D C}^{\mathrm{i}}}{\longrightarrow} \mathbf{S}-\mathbf{F C}^{C}$, which is given by $H_{D C}^{\mathrm{i}}\left(\mathscr{K}_{1} \stackrel{f}{\rightarrow} \mathscr{K}_{2}\right)=\left(L^{G_{1}}, L^{M_{1}}, L, \widehat{I}_{1}\right) \stackrel{\left(\alpha, \beta, 1_{L}\right)}{\longrightarrow}$ $\left(L^{G_{2}}, L^{M_{2}}, L, \widehat{I}_{2}\right)$, where

$$
\widehat{I}_{j}(s, t)= \begin{cases}\top_{L}, & (s, t) \text { is a formal concept of } \mathscr{K}_{j} \\ \perp_{L}, & \text { otherwise. }\end{cases}
$$

Proof. We have to show that the functor is correct on morphisms. Given $s_{1} \in L^{G_{1}}$ and $t_{2} \in L^{M_{2}}$, it follows that $\widehat{I}_{1}\left(s_{1}, \beta^{o p}\left(t_{2}\right)\right)=\mathrm{T}_{L}$ iff $\left(s_{1}, \beta^{o p}\left(t_{2}\right)\right)$ is a formal concept of $\mathscr{K}_{1}$ iff $\left(\alpha\left(s_{1}\right), t_{2}\right)$ is a formal concept of $\mathscr{K}_{2}$ iff $\widehat{I}_{2}\left(\alpha\left(s_{1}\right), t_{2}\right)=\mathrm{T}_{L}$, where the second "iff" uses Theorem 45(3).

In the following, we make the functor of Theorem 62 into an embedding. To achieve the goal, we impose a restriction on the chosen L-algebra $L$.

Definition 63. An $\mathbf{L}$-algebra $L$ is called quasistrictly rightsided (qsrs-algebra, for short) provided that $a \leqslant\left(\top_{L} \rightarrow{ }_{l} a\right) \otimes$ $\mathrm{T}_{L}$ for every $a \in L$.

Since every quantale $Q$ has the property $\left(T_{Q} \rightarrow{ }_{l} q\right) \otimes T_{Q} \leqslant$ $q$ for every $q \in Q$ (use Example 21(2)), every qsrs-algebra $L$ satisfies $\left(\mathrm{T}_{L} \rightarrow{ }_{l} a\right) \otimes \mathrm{T}_{L}=a$ for every $a \in L$. Additionally, if $Q$ is a strictly right-sided quantale (recall Definition 9), then, for every $q \in Q, \mathrm{~T}_{L} \rightarrow{ }_{l} q=q$ yields $\left(\mathrm{T}_{L} \rightarrow{ }_{l} q\right) \otimes \mathrm{T}_{L}=\mathrm{T}_{L} \rightarrow{ }_{l} q=$ $q$. As a result, every $L$-algebra $L$ extending a strictly rightsided quantale $Q$ is a qsrs-algebra, but not vice versa.

Theorem 64. For every qsrs-algebra $L$, the functor $L-\mathbf{F C}_{\mathfrak{i}}^{D M R} \stackrel{H_{D C}^{i}}{\longrightarrow} \mathbf{S}-\mathbf{F C}^{C}$ is a nonfull embedding.

Proof. It will be enough to show that the functor $H_{D C}^{\mathrm{i}}$ is injective on objects. Given $\mathscr{K}_{1}, \mathscr{K}_{2}$ in $L-\mathbf{F C}_{\mathbf{i}}^{D M R}$ such that $H_{D C}^{\mathrm{i}}\left(\mathscr{K}_{1}\right)=H_{D C}^{\mathrm{i}}\left(\mathscr{K}_{2}\right)$, it follows that $\left(L^{G_{1}}, L^{M_{1}}, L, \widehat{I}_{1}\right)=\left(L^{G_{2}}, L^{M_{2}}, L, \widehat{I}_{2}\right)$ and, therefore, $G_{1}=G_{2}=G, M_{1}=M_{2}=M$ and $\widehat{I}_{1}=\widehat{I}_{2}$. To show that $I_{1}=I_{2}$, we assume that $I_{1} \neq I_{2}$. Then there exist some $g \in G$ and $m \in M$ such that $I_{1}(g, m) \nless I_{2}(g, m)$. As a consequence, we obtain that $\perp_{L} \neq T_{L}$. The properties of Galois connections of Theorem 19 imply that $H_{1} \circ K_{1} \circ H_{1}\left(\chi_{\{g\}}^{\top_{L}}\right)=H_{1}\left(\chi_{\{g\}}^{\top_{L}}\right)$, and, therefore, $\left(K_{1} \circ H_{1}\left(\chi_{\{g\}}^{\top_{L}}\right), H_{1}\left(\chi_{\{g\}}^{\top_{L}}\right)\right)$ is a formal concept of $\mathscr{K}_{1}$. As a consequence, $\widehat{I}_{1}\left(K_{1} \circ H_{1}\left(\chi_{\{g\}}^{\top_{L}}\right), H_{1}\left(\chi_{\{g\}}^{\top_{L}}\right)\right)=\mathrm{\top}_{L}$ and, thus, $\widehat{I}_{2}\left(K_{1} \circ H_{1}\left(\chi_{\{g\}}^{\top_{L}}\right), H_{1}\left(\chi_{\{g\}}^{\top_{L}}\right)\right)=\top_{L}$; that is, $\left(K_{1} \circ\right.$ $\left.H_{1}\left(\chi_{\{g\}}^{\top_{L}}\right), H_{1}\left(\chi_{\{g\}}^{\top_{L}}\right)\right)$ is a formal concept of $\mathscr{K}_{2}$. It follows that $\chi_{\{g\}}^{\top_{L}} \leqslant K_{1} \circ H_{1}\left(\chi_{\{g\}}^{\top_{L}}\right)=K_{2} \circ H_{1}\left(\chi_{\{g\}}^{\top_{L}}\right)$ and, therefore, $\top_{L}=\chi_{\{g\}}^{\top_{L}}(g) \leqslant\left(K_{1} \circ H_{1}\left(\chi_{\{g\}}^{\top_{L}}\right)\right)(g)=\left(K_{2} \circ\right.$ $\left.H_{1}\left(\chi_{\{g\}}^{\top_{L}}\right)\right)(g)=\bigwedge_{m^{\prime} \in M}\left(\left(H_{1}\left(\chi_{\{g\}}^{\top_{L}}\right)\right)\left(m^{\prime}\right) \rightarrow{ }_{r} I_{2}\left(g, m^{\prime}\right)\right)=$ $\bigwedge_{m^{\prime} \in M}\left(\left(\bigwedge_{g^{\prime} \in G}\left(\chi_{\{g\}}^{\top_{L}}\left(g^{\prime}\right) \rightarrow{ }_{l} I_{1}\left(g^{\prime}, m^{\prime}\right)\right)\right) \rightarrow{ }_{r} I_{2}\left(g, m^{\prime}\right)\right)=$ $\bigwedge_{m^{\prime} \in M}\left(\left(\mathrm{\top}_{L} \rightarrow{ }_{l} I_{1}\left(g, m^{\prime}\right)\right) \rightarrow{ }_{r} I_{2}\left(g, m^{\prime}\right)\right) \leqslant\left(\mathrm{T}_{L} \rightarrow{ }_{l} I_{1}(g, m)\right) \rightarrow{ }_{r}$ $I_{2}(g, m)$. As a result, $I_{1}(g, m) \stackrel{(\dagger)}{\leqslant}\left(\top_{L} \rightarrow{ }_{l} I_{1}(g, m)\right) \otimes \top_{L} \leqslant$ $I_{2}(g, m)$, where $(\dagger)$ uses the definition of qsrs-algebras, and, therefore, $I_{1}(g, m) \leqslant I_{2}(g, m)$, which contradicts our assumption $I_{1}(g, m) \nless I_{2}(g, m)$.
To show that the functor is nonfull, we let $\mathbf{L}=$ Quant and $L=([0,1], \bigvee, \bigwedge)$, that is, taking the unit interval $[0,1]$, with its standard lattice-theoretic structure. Since $L$ is clearly strictly two-sided, it is quasistrictly right-sided. Define a map $|L| \stackrel{\varphi}{\rightarrow}|L|$ by

$$
\varphi(a)= \begin{cases}\frac{3}{2} \cdot a, & a \in\left[0, \frac{1}{2}\right], \\ \frac{1}{2} \cdot a+\frac{1}{2}, & a \in\left(\frac{1}{2}, 1\right] .\end{cases}
$$

It follows that $L \stackrel{\varphi}{\rightarrow} L$ is an STSQuant-isomorphism, which is different from $1_{L}$. Define now a lattice-valued formal context $\mathscr{K}$ as $G=M=\{*\}$ and $I=\underline{\top}_{L}$. It is easy to see that $H_{D C}^{\mathfrak{i}}(\mathscr{K}) \stackrel{f=\left(1_{L^{G}}, 1_{L^{M}}, \varphi^{o p}\right)}{\longrightarrow} H_{D C}^{\mathfrak{i}}(\mathscr{K})$ is an $\mathbf{S}-\mathbf{F C}^{C}$-morphism, since $\varphi(a)=\mathrm{T}_{L}$ (resp., $\varphi(a)=\perp_{L}$ ) iff $a=\mathrm{T}_{L}$ (resp., $a=\perp_{L}$ ). Additionally, the morphism is clearly not in the image of the functor $H_{D C}^{\mathrm{i}}$.

To continue, we provide the second of the claimed embeddings. As usual, we begin by singling out a particular fixed-basis subcategory of the category S-FC ${ }^{D M R}$.

Definition 65. Given an L-algebra $L, L-\mathbf{F C}_{\text {orp }}^{D M R}$ is the (nonfull) subcategory of the category $L-F C^{D M R}$, with the same objects, whose morphisms $\mathscr{K}_{1} \stackrel{f}{\rightarrow} \mathscr{K}_{2}$ satisfy the property that the maps $L^{G_{1}} \stackrel{\alpha}{\rightarrow} L^{G_{2}}, L^{M_{2}} \stackrel{\beta^{o p}}{\longrightarrow} L^{M_{1}}$ are order-preserving.

The reader should notice that "orp" in $L-\mathrm{FC}_{\text {orp }}^{D M R}$ stands for "order-preserving."

Theorem 66. There exists the functor $L-\mathbf{F C}_{\text {orp }}^{D M R} \stackrel{H_{D C}^{\text {orp }}}{\longrightarrow} \mathbf{S}-\mathbf{F C}^{C}$, which is given by $H_{D C}^{\text {orp }}\left(\mathscr{K}_{1} \stackrel{f}{\rightarrow} \mathscr{K}_{2}\right)=\left(L^{G_{1}}, L^{M_{1}}, L, \widehat{I}_{1}\right) \stackrel{\left(\alpha, \beta, 1_{L}\right)}{\longrightarrow}$ $\left(L^{G_{2}}, L^{M_{2}}, L, \widehat{I}_{2}\right)$, where

$$
\widehat{I}_{j}(s, t)= \begin{cases}\top_{L}, & (s, t) \text { is a formal preconcept of } \mathscr{K}_{j} \\ \perp_{L}, & \text { otherwise. }\end{cases}
$$

Proof. We have to show that the functor is correct on morphisms. Given $s_{1} \in L^{G_{1}}$ and $t_{2} \in L^{M_{2}}$, it follows that $\widehat{I}_{1}\left(s_{1}, \beta^{o p}\left(t_{1}\right)\right)=\mathrm{T}_{L}$ iff $\left(s_{1}, \beta^{o p}\left(t_{1}\right)\right)$ is a formal preconcept of $\mathscr{K}_{1}$ iff $\left(\alpha\left(s_{1}\right), t_{2}\right)$ is a formal preconcept of $\mathscr{K}_{2}$ iff $\widehat{I}_{2}\left(\alpha\left(s_{1}\right), t_{2}\right)=\top_{L}$, where the second "iff" uses Theorem 45(5).

The machinery of Theorem 64 does well enough in this modified case as well.

Theorem 67. For every qsrs-algebra $L$, the functor $L-\mathbf{F C}_{\text {orp }}^{D M R} \stackrel{H_{D C}^{o r p}}{\longrightarrow} \mathbf{S}-\mathrm{FC}^{C}$ is a nonfull embedding.

Proof. The proof starts precisely as that of Theorem 64. To show that $I_{1}=I_{2}$, we again assume the existence of some $g \in$ $G$ and $m \in M$ such that $I_{1}(g, m) \nless I_{2}(g, m)$. The properties of 
Galois connections (Theorem 19) provide $\chi_{\{g\}}^{\top_{L}} \leqslant K_{1} \circ H_{1}\left(\chi_{\{g\}}^{\top_{L}}\right)$, which implies that $\left(\chi_{\{g\}}^{\top_{L}}, H_{1}\left(\chi_{\{g\}}^{\top_{L}}\right)\right)$ is a formal preconcept of $\mathscr{K}_{1}$. It follows that $\widehat{I}_{1}\left(\chi_{\{g\}}^{\top_{L}}, H_{1}\left(\chi_{\{g\}}^{\top_{L}}\right)\right)=\top_{L}$ and, therefore, $\widehat{I}_{2}\left(\chi_{\{g\}}^{\top_{L}}, H_{1}\left(\chi_{\{g\}}^{\top_{L}}\right)\right)=\top_{L}$; that is, $\left(\chi_{\{g\}}^{\top_{L}}, H_{1}\left(\chi_{\{g\}}^{\top_{L}}\right)\right)$ is a formal preconcept of $\mathscr{K}_{2}$, and, as a result, $\chi_{\{g\}}^{\top_{L}} \leqslant K_{2} \circ H_{1}\left(\chi_{\{g\}}^{\top_{L}}\right)$. Similar to the proof of Theorem 64 , one obtains that $I_{1}(g, m) \leqslant$ $I_{2}(g, m)$, thereby getting a contradiction with the assumption $I_{1}(g, m) \nless I_{2}(g, m)$.

The nonfullness part follows the steps of the proof of Theorem 64.

To continue, we show yet another one of the claimed embeddings, which never requires singling out a particular subcategory of the category $L-F^{D M R}$ (but is still fixed-basis).

Theorem 68. There exists the functor $L-\mathbf{F C}^{D M R} \stackrel{H_{D C}}{\longrightarrow} \mathbf{S}-\mathrm{FC}^{C}$, which is given by $H_{D C}\left(\mathscr{K}_{1} \stackrel{f}{\rightarrow} \mathscr{K}_{2}\right)=\left(L^{G_{1}}, L^{M_{1}}, L, \widehat{I}_{1}\right) \stackrel{\left(\alpha, \beta, 1_{L}\right)}{\longrightarrow}$ $\left(L^{G_{2}}, L^{M_{2}}, L, \widehat{I}_{2}\right)$, where

$$
\widehat{I}_{j}(s, t)= \begin{cases}\top_{L}, & (s, t) \text { is a formal protoconcept of } \mathscr{K}_{j} \\ \perp_{L}, & \text { otherwise. }\end{cases}
$$

Proof. We have to show that the functor is correct on morphisms. Given $s_{1} \in L^{G_{1}}$ and $t_{2} \in \mathrm{L}^{M_{2}}$, it follows that $\widehat{I}_{1}\left(s_{1}, \beta^{o p}\left(t_{1}\right)\right)=\mathrm{T}_{L}$ iff $\left(s_{1}, \beta^{o p}\left(t_{1}\right)\right)$ is a formal protoconcept of $\mathscr{K}_{1}$ iff $\left(\alpha\left(s_{1}\right), t_{2}\right)$ is a formal protoconcept of $\mathscr{K}_{2}$ iff $\widehat{I}_{2}\left(\alpha\left(s_{1}\right), t_{2}\right)=\mathrm{T}_{L}$, where the second "iff" uses Theorem 45(4).

The machinery of Theorem 64 does well even in this extended setting.

Theorem 69. For every qsrs-algebra $L$, the functor $L-F_{C} C^{D M R} \stackrel{H_{D C}}{\longrightarrow} \mathbf{S}-\mathbf{F C}^{C}$ is a nonfull embedding.

Proof. The proof starts precisely as that of Theorem 64. To show that $I_{1}=I_{2}$, we again assume the existence of some $g \in G$ and $m \in M$ such that $I_{1}(g, m) \nless I_{2}(g, m)$. Since $K_{1} \circ H_{1}\left(\chi_{\{g\}}^{\top_{L}}\right)=K_{1} \circ H_{1}\left(\chi_{\{g\}}^{\top_{L}}\right),\left(\chi_{\{g\}}^{\top_{L}}, H_{1}\left(\chi_{\{g\}}^{\top_{L}}\right)\right)$ is a formal protoconcept of $\mathscr{K}_{1}$. It follows that $\widehat{I}_{1}\left(\chi_{\{g\}}^{\top_{L}}, H_{1}\left(\chi_{\{g\}}^{\top_{L}}\right)\right)=\mathrm{T}_{L}$ and, therefore, $\widehat{I}_{2}\left(\chi_{\{g\}}^{\top_{L}}, H_{1}\left(\chi_{\{g\}}^{\top_{L}}\right)\right)=\top_{L}$, that is, $\left(\chi_{\{g\}}^{\top_{L}}, H_{1}\left(\chi_{\{g\}}^{\top_{L}}\right)\right)$ is a formal protoconcept of $\mathscr{K}_{2}$, and, as a result, $\chi_{\{g\}}^{\top_{L}} \leqslant K_{2}$ 。 $H_{2}\left(\chi_{\{g\}}^{\top_{L}}\right)=K_{2} \circ H_{1}\left(\chi_{\{g\}}^{\top_{L}}\right)$. Similar to the proof of Theorem 64, one obtains that $I_{1}(g, m) \leqslant I_{2}(g, m)$, thereby getting a contradiction with the assumption $I_{1}(g, m) \nless I_{2}(g, m)$.

The nonfullness part follows the steps of the proof of Theorem 64.

Theorems 71 and 72 show the last of the claimed embeddings; this embedding does require a particular subcategory of $L-\mathrm{FC}^{D M R}$.

Definition 70. Given an L-algebra $L, L-\mathbf{F C}_{r f p}^{D M R}$ is the (nonfull) subcategory of the category $L-F C^{D M R}$, with the same objects, whose morphisms $\mathscr{K}_{1} \stackrel{f}{\rightarrow} \mathscr{K}_{2}$ satisfy the property that the maps $L^{G_{1}} \stackrel{\alpha}{\rightarrow} L^{G_{2}}, L^{M_{2}} \stackrel{\beta^{o p}}{\longrightarrow} L^{M_{1}}$ reflect fixed points.

The reader should notice that " $r f p$ " in $L-\mathbf{F C}_{r f p}^{D M R}$ stands for "reflect fixed points."

Theorem 71. There exists the functor $L-\mathbf{F C}_{r f p}^{D M R} \stackrel{H_{D C}^{r f p}}{\longrightarrow} \mathbf{S}-\mathbf{F C}$, which is given by $H_{D C}^{r f p}\left(\mathscr{K}_{1} \stackrel{f}{\rightarrow} \mathscr{K}_{2}\right)=\left(L^{G_{1}}, L^{M_{1}}, L, \widehat{I}_{1}\right) \stackrel{\left(\alpha, \beta, 1_{L}\right)}{\longrightarrow}$ $\left(L^{G_{2}}, L^{M_{2}}, L, \widehat{I}_{2}\right)$, where

$$
\widehat{I}_{j}(s, t)= \begin{cases}\top_{L}, & (s, t) \text { is a formal concept of } \mathscr{K}_{j} \\ \perp_{L}, & \text { otherwise. }\end{cases}
$$

Proof. We have to show that the functor is correct on morphisms. Given $s_{1} \in L^{G_{1}}$ and $t_{2} \in L^{M_{2}}$, it follows that $\widehat{I}_{1}\left(s_{1}, \beta^{o p}\left(t_{1}\right)\right)=\mathrm{T}_{L}$ iff $\left(s_{1}, \beta^{o p}\left(t_{1}\right)\right)$ is a formal concept of $\mathscr{K}_{1}$ iff $\left(\alpha\left(s_{1}\right), t_{2}\right)$ is a formal concept of $\mathscr{K}_{2}$ iff $\widehat{I}_{2}\left(\alpha\left(s_{1}\right), t_{2}\right)=\mathrm{T}_{L}$, where the second "iff" uses Theorem 45(2).

The embedding property of the just introduced functor is easy to get.

Theorem 72. For every qsrs-algebra $L$, the functor $L-\mathbf{F C}_{r f p}^{D M R} \stackrel{H_{D C}^{r f p}}{\longrightarrow} \mathbf{S}-\mathbf{F C}^{C}$ is a nonfull embedding.

Proof. The proof follows the steps of that of Theorem 64 .

In view of the last claim of Theorem 45, the category $L-\mathbf{F C}_{\mathfrak{i}}^{D M R}$ is a (nonfull) subcategory of the category $L-\mathbf{F C}_{r f p}^{D M R}$. Thus, the functor $H_{D M}^{\mathrm{i}}$ is the restriction of the functor $H_{D M}^{r f p}$ to the category $L-\mathbf{F C}_{\mathfrak{i}}^{D M R}$.

As a last remark, we notice that in every unital quantale $Q,\left(1_{\mathrm{Q}} \rightarrow{ }_{l} q\right) \otimes 1_{\mathrm{Q}}=1_{\mathrm{Q}} \rightarrow{ }_{l} q=q$ for every $q \in Q$. In particular, all the results in this subsection can be done in a more simple way, assuming that $\mathbf{L}$ extends the variety UQuant of unital quantales and then making use of the unit $1_{L}$ instead of the top element $\top_{L}$ of the given $\mathrm{L}$-algebra $L$. In view of the previously mentioned property of unital quantales, the respective functors are then embeddings, without any additional requirement on the $\mathbf{L}$-algebra $L$.

5.1.6. From $\mathbf{S}-\mathbf{F} \mathbf{C}^{D M R}$ to $\mathbf{S}-\mathbf{F C}_{m}^{C}$. The reader can easily show (as a simple exercise) that the functors from the previous subsection can be used in case of the categories S-FC ${ }^{D M R}$ and S-FC ${ }_{m}^{C}$ as well, providing similar results. In particular, all the nonfullness examples work also in the new setting.

5.2. S-FC ${ }^{G W}$ and $\mathbf{S}-\mathbf{F C}_{m}^{G W}$ versus $\mathbf{S}-\mathbf{F} \mathbf{C}^{D M R}$ and $\mathbf{S}-\mathbf{F C}_{a}^{D M R}$. In this subsection, we consider possible functorial links between the categories $\mathbf{S}-\mathbf{F C}{ }^{G W}$ and $\mathbf{S}-\mathbf{F C} \boldsymbol{m}_{m}^{G W}$, on one side, and the categories S-FC ${ }^{D M R}$ and $\mathbf{S}-\mathbf{F C}_{a}^{D M R}$, on the other. The obtained functors can be easily made into embeddings, employing the machinery of Section 5.1 related to the functors from the constructed subcategories of the category S-FC ${ }^{C}$ (resp., 
S-FC $C_{m}^{C}$ ) to the category $\mathbf{S}-\mathbf{F C}^{D M R}$ (resp., S-FC $\mathbf{C}_{a}^{D M R}$ ). In particular, assuming that $\mathbf{L}$ extends the variety $\mathbf{U Q u a n t}$ of unital quantales, whereas $\mathbf{S}-\mathbf{F C} \mathbf{f}_{\dagger *}^{G W}$ is the domain of the newly constructed functor (see the results of following subsections), where $\dagger$ is ether "' or " $m$," we can define the following two subcategories.

Definition 73. $\mathbf{S}-\mathbf{F C}_{\dagger * *}^{G W}$ (resp., $\mathbf{S}-\mathbf{F C} \mathbf{C}_{\dagger * \bullet}^{G W}$ ) is the full subcategory of the category $\mathbf{S}-\mathbf{F C}_{\dagger_{*}}^{G W}$, whose objects $\mathscr{K}$ are such that $G$ is nonempty (resp., $M$ is nonempty) and, moreover, $1_{L} \neq \perp_{L}$.

Employing the technique of Section 5.1, we can easily get that the restriction of the respective constructed functor to the previously mentioned two categories provides two embeddings. To save the space, therefore, we will not mention the just discussed developments explicitly.

5.2.1. From $\mathbf{S}-\mathbf{F C}_{m}^{G W}$ to $\mathbf{S}-\mathbf{F C}_{a}^{D M R}$. In this subsection, we construct a functor from a particular subcategory of the category $\mathbf{S}-\mathbf{F} \mathbf{C}_{m}^{G W}$ to the category $\mathbf{S}-\mathbf{F C}_{a}^{D M R}$. To begin with, we provide the definition of the subcategory in question.

Definition 74. S-FC $\mathbf{F}_{m *}^{G W}$ is the (nonfull) subcategory of the category $\mathbf{S}-\mathbf{F C}_{m}^{G W}$, with the same objects, whose morphisms $\mathscr{K}_{1} \stackrel{f}{\rightarrow} \mathscr{K}_{2}$ are such that the maps $G_{1} \stackrel{\alpha}{\rightarrow} G_{2}$ and $M_{1} \stackrel{\beta}{\rightarrow} M_{2}$ are surjective, whereas the $\mathbf{S}$-morphism $L_{1} \stackrel{\varphi}{\rightarrow} L_{2}$ preserves $\bigwedge, \rightarrow_{l}$ and $\rightarrow_{r}$.

For the new category in hand, we can construct the following functor.

Theorem 75. There exists the functor $\mathbf{S}-\mathrm{FC}_{m *}^{G W} \stackrel{H_{\mathrm{GmDa}}}{\longrightarrow}$ S-FC ${ }_{a}^{D M R}$, which is defined by the formula $H_{G m D a}\left(\mathscr{K}_{1} \stackrel{f}{\rightarrow}\right.$ $\left.\mathscr{K}_{2}\right)=\mathscr{K}_{1} \stackrel{\left((\alpha, \varphi)^{\rightarrow},(\beta, \varphi)^{\rightarrow}\right)}{\longrightarrow} \mathscr{K}_{2}$.

Proof. To show that the functor is correct on morphisms, we verify commutativity of the diagrams

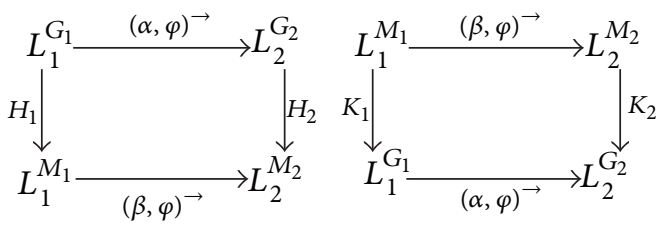

For the left-hand diagram, notice that given $s \in L_{1}^{G_{1}}$ and $m_{2} \in M_{2}$,

$$
\begin{aligned}
((\beta, \varphi) & \left.\rightarrow \circ H_{1}(s)\right)\left(m_{2}\right) \\
& =\varphi\left(\bigvee_{\beta\left(m_{1}\right)=m_{2}}\left(H_{1}(s)\right)\left(m_{1}\right)\right)
\end{aligned}
$$

$$
\begin{aligned}
& =\varphi\left(\bigvee_{\beta\left(m_{1}\right)=m_{2}}\left(\bigwedge_{g_{1} \in G_{1}}\left(s\left(g_{1}\right) \longrightarrow I_{1}\left(g_{1}, m_{1}\right)\right)\right)\right) \\
& \stackrel{(\oplus)}{=} \bigvee_{\beta\left(m_{1}\right)=m_{2}} \bigwedge_{g_{1} \in G_{1}}\left(\varphi \circ s\left(g_{1}\right) \longrightarrow l \varphi \circ I_{1}\left(g_{1}, m_{1}\right)\right) \\
& \stackrel{(\dagger)}{=} \bigvee_{\beta\left(m_{1}\right)=m_{2}} \bigwedge_{g_{1} \in G_{1}}\left(\varphi \circ s\left(g_{1}\right) \longrightarrow_{l} I_{2}\left(\alpha\left(g_{1}\right), \beta\left(m_{1}\right)\right)\right) \\
& =\bigvee_{\beta\left(m_{1}\right)=m_{2}} \bigwedge_{g_{1} \in G_{1}}\left(\varphi \circ s\left(g_{1}\right) \longrightarrow I_{l} I_{2}\left(\alpha\left(g_{1}\right), m_{2}\right)\right) \\
& \stackrel{(\dagger)}{=} \bigwedge_{g_{1} \in G_{1}}\left(\varphi \circ s\left(g_{1}\right) \longrightarrow{ }_{l} I_{2}\left(\alpha\left(g_{1}\right), m_{2}\right)\right) \\
& =\bigwedge_{g_{2} \in G_{2}} \bigwedge_{\alpha\left(g_{1}\right)=g_{2}}\left(\varphi \circ s\left(g_{1}\right) \longrightarrow I_{2}\left(g_{2}, m_{2}\right)\right) \\
& =\bigwedge_{g_{2} \in G_{2}}\left(\left(\bigvee_{\alpha\left(g_{1}\right)=g_{2}} \varphi \circ s\left(g_{1}\right)\right) \longrightarrow{ }_{l} I_{2}\left(g_{2}, m_{2}\right)\right) \\
& =\bigwedge_{g_{2} \in G_{2}}\left(\varphi\left(\bigvee_{\alpha\left(g_{1}\right)=g_{2}} s\left(g_{1}\right)\right) \longrightarrow_{l} I_{2}\left(g_{2}, m_{2}\right)\right) \\
& =\bigwedge_{g_{2} \in G_{2}}\left(((\alpha, \varphi) \rightarrow(s))\left(g_{2}\right) \longrightarrow{ }_{l} I_{2}\left(g_{2}, m_{2}\right)\right) \\
& =\left(H_{2} \circ(\alpha, \varphi) \rightarrow(s)\right)\left(m_{2}\right) \text {, }
\end{aligned}
$$

where $(\dagger)$ uses Definition 74, whereas $(\dagger \dagger)$ employs the fact that $\mathscr{K}_{1} \stackrel{f}{\rightarrow} \mathscr{K}_{2}$ is a morphism of $\mathbf{S}-\mathbf{F C}_{m}^{G W}$.

For the right-hand diagram, notice that given $t \in L_{1}^{M_{1}}$ and $g_{2} \in G_{2}$,

$$
\begin{aligned}
& \left(K_{2} \circ(\beta, \varphi) \rightarrow(t)\right)\left(g_{2}\right) \\
& =\bigwedge_{m_{2} \in M_{2}}\left(((\beta, \varphi) \rightarrow(t))\left(m_{2}\right) \longrightarrow_{r} I_{2}\left(g_{2}, m_{2}\right)\right) \\
& \quad=\bigwedge_{m_{2} \in M_{2}}\left(\varphi\left(\bigvee_{\beta\left(m_{1}\right)=m_{2}} t\left(m_{1}\right)\right) \rightarrow_{r} I_{2}\left(g_{2}, m_{2}\right)\right) \\
& =\bigwedge_{m_{2} \in M_{2}}\left(\left(\bigvee_{\beta\left(m_{1}\right)=m_{2}} \varphi \circ t\left(m_{1}\right)\right) \longrightarrow_{r} I_{2}\left(g_{2}, m_{2}\right)\right) \\
& \quad=\bigwedge_{m_{2} \in M_{2}} \bigwedge_{\beta\left(m_{1}\right)=m_{2}}\left(\varphi \circ t\left(m_{1}\right) \longrightarrow I_{r} I_{2}\left(g_{2}, m_{2}\right)\right)
\end{aligned}
$$




$$
\begin{aligned}
& =\bigwedge_{m_{1} \in M_{1}}\left(\varphi \circ t\left(m_{1}\right) \longrightarrow_{r} I_{2}\left(g_{2}, \beta\left(m_{1}\right)\right)\right) \\
& \stackrel{(\dagger)}{=} \bigvee_{\alpha\left(g_{1}\right)=g_{2}} \bigwedge_{m_{1} \in M_{1}}\left(\varphi \circ t\left(m_{1}\right) \longrightarrow_{r} I_{2}\left(\alpha\left(g_{1}\right), \beta\left(m_{1}\right)\right)\right) \\
& \stackrel{(+\dagger)}{=} \bigvee_{\alpha\left(g_{1}\right)=g_{2}} \bigwedge_{m_{1} \in M_{1}}\left(\varphi \circ t\left(m_{1}\right) \longrightarrow_{r} \varphi \circ I_{1}\left(g_{1}, m_{1}\right)\right) \\
& \stackrel{(\dagger)}{=} \varphi\left(\bigvee_{\alpha\left(g_{1}\right)=g_{2}} \bigwedge_{m_{1} \in M_{1}}\left(t\left(m_{1}\right) \longrightarrow_{r} I_{1}\left(g_{1}, m_{1}\right)\right)\right) \\
& =\varphi\left(\bigvee_{\alpha\left(g_{1}\right)=g_{2}}\left(K_{1}(t)\right)\left(g_{1}\right)\right) \\
& =\left((\alpha, \varphi) \rightarrow \circ K_{1}(t)\right)\left(g_{2}\right),
\end{aligned}
$$

where $(\dagger)$ uses Definition 74, whereas $(\dagger \dagger)$ employs the fact that $\mathscr{K}_{1} \stackrel{f}{\rightarrow} \mathscr{K}_{2}$ is a morphism of $\mathbf{S}-\mathbf{F C}_{a}^{G W}$.

5.2.2. From $\mathbf{S}-\mathbf{F C}_{m}^{G W}$ to $\mathbf{S}-\mathbf{F C}^{D M R}$. In this subsection, we construct a functor from a particular subcategory of the category $\mathbf{S}-\mathbf{F} \mathbf{C}_{m}^{G W}$ to the category $\mathbf{S}-\mathbf{F C}^{D M R}$. To begin with, we provide the definition of the subcategory in question.

Definition 76. S-FC $\mathbf{C}_{m *}^{G W}$ is the (nonfull) subcategory of the category $\mathbf{S}-\mathbf{F} \mathbf{C}_{m}^{G W}$, with the same objects, whose morphisms $\mathscr{K}_{1} \stackrel{f}{\rightarrow} \mathscr{K}_{2}$ are such that the maps $G_{1} \stackrel{\alpha}{\rightarrow} G_{2}$ and $M_{1} \stackrel{\beta}{\rightarrow}$ $M_{2}$ are surjective, whereas the $\mathbf{S}$-morphism $L_{1} \stackrel{\varphi}{\rightarrow} L_{2}$ is an isomorphism.

Theorem 77. There exists the functor $\mathbf{S}-\mathrm{FC}_{m *}^{G W} \stackrel{\mathrm{H}_{\mathrm{GmD}}}{\longrightarrow}$ S-FC ${ }^{D M R}$, which is defined by the formula $H_{G m D}\left(\mathscr{K}_{1} \stackrel{f}{\rightarrow}\right.$ $\left.\mathscr{K}_{2}\right)=\mathscr{K}_{1} \stackrel{\left((\alpha, \varphi)^{\rightarrow},\left((\beta, \varphi)^{\vdash \cdots}\right)^{o p}\right)}{\longrightarrow} \mathscr{K}_{2}$.

Proof. To show that the functor is correct on morphisms, we verify commutativity of the diagrams

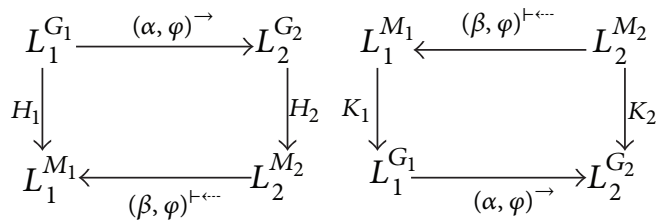

For the left-hand diagram, notice that given $s \in L_{1}^{G_{1}}$ and $m_{1} \in M_{1}$,

$$
\begin{aligned}
& \left((\beta, \varphi)^{\vdash \cdots} \circ H_{2} \circ(\alpha, \varphi)^{\rightarrow}(s)\right)\left(m_{1}\right) \\
& \quad=\varphi^{\vdash} \circ\left(H_{2}\left((\alpha, \varphi)^{\rightarrow}(s)\right)\right) \circ \beta\left(m_{1}\right)
\end{aligned}
$$

$$
\begin{aligned}
& =\varphi^{\vdash}\left(\bigwedge_{g_{2} \in G_{2}}\left(((\alpha, \varphi) \rightarrow(s))\left(g_{2}\right) \longrightarrow_{l} I_{2}\left(g_{2}, \beta\left(m_{1}\right)\right)\right)\right) \\
& =\varphi^{\vdash}\left(\bigwedge_{g_{2} \in G_{2}}\left(\varphi\left(\bigvee_{\alpha\left(g_{1}\right)=g_{2}} s\left(g_{1}\right)\right) \longrightarrow_{l} I_{2}\left(g_{2}, \beta\left(m_{1}\right)\right)\right)\right) \\
& =\varphi^{\vdash}\left(\bigwedge_{g_{2} \in G_{2}}\left(\left(\bigvee_{\alpha\left(g_{1}\right)=g_{2}} \varphi \circ s\left(g_{1}\right)\right) \longrightarrow_{l} I_{2}\left(g_{2}, \beta\left(m_{1}\right)\right)\right)\right) \\
& =\varphi^{\vdash}\left(\bigwedge_{g_{2} \in G_{2}}\left(\bigwedge_{\alpha\left(g_{1}\right)=g_{2}}\left(\varphi \circ s\left(g_{1}\right) \longrightarrow_{l} I_{2}\left(g_{2}, \beta\left(m_{1}\right)\right)\right)\right)\right) \\
& =\varphi^{\vdash}\left(\bigwedge_{g_{1} \in G_{1}}\left(\varphi \circ s\left(g_{1}\right) \longrightarrow I_{l} I_{2}\left(\alpha\left(g_{1}\right), \beta\left(m_{1}\right)\right)\right)\right) \\
& \stackrel{(\dagger)}{=} \varphi^{\vdash}\left(\bigwedge_{g_{1} \in G_{1}}\left(\varphi \circ s\left(g_{1}\right) \longrightarrow \longrightarrow_{l} \varphi I_{1}\left(g_{1}, m_{1}\right)\right)\right) \\
& \stackrel{(\dagger+)}{=} \varphi^{\vdash} \circ \varphi\left(\bigwedge_{g_{1} \in G_{1}}\left(s\left(g_{1}\right) \longrightarrow{ }_{l} I_{1}\left(g_{1}, m_{1}\right)\right)\right) \\
& \stackrel{(\dagger+)}{=} \bigwedge_{g_{1} \in G_{1}}\left(s\left(g_{1}\right) \longrightarrow{ }_{l} I_{1}\left(g_{1}, m_{1}\right)\right) \\
& =\left(H_{1}(s)\right)\left(m_{1}\right),
\end{aligned}
$$

where $(\dagger)$ uses the fact that $\mathscr{K}_{1} \stackrel{f}{\rightarrow} \mathscr{K}_{2}$ is a morphism of S-FC ${ }_{m}^{G W}$, whereas (††) employs Definition 76.

For the right-hand diagram, notice that given $t \in L_{2}^{M_{2}}$ and $g_{2} \in G_{2}$,

$$
\begin{aligned}
((\alpha, \varphi) \rightarrow & \left.\circ K_{1} \circ(\beta, \varphi)^{\vdash \cdots}(t)\right)\left(g_{2}\right) \\
& =\left((\alpha, \varphi) \rightarrow \circ\left(K_{1}\left(\varphi^{\vdash} \circ t \circ \beta\right)\right)\right)\left(g_{2}\right) \\
& =\varphi\left(\bigvee_{\alpha\left(g_{1}\right)=g_{2}}\left(K_{1}\left(\varphi^{\vdash} \circ t \circ \beta\right)\right)\left(g_{1}\right)\right) \\
& =\varphi\left(\bigvee_{\alpha\left(g_{1}\right)=g_{2}}\left(\bigwedge_{m_{1} \in M_{1}}\left(\varphi^{\vdash} \circ t \circ \beta\left(m_{1}\right) \longrightarrow_{r} I_{1}\left(g_{1}, m_{1}\right)\right)\right)\right) \\
& \stackrel{(\dagger)}{=} \bigvee_{\alpha\left(g_{1}\right)=g_{2}} \bigwedge_{m_{1} \in M_{1}}\left(\varphi \circ \varphi^{\vdash} \circ t \circ \beta\left(m_{1}\right) \longrightarrow_{r} \varphi \circ I_{1}\left(g_{1}, m_{1}\right)\right) \\
& \stackrel{(\dagger \dagger)}{=} \bigvee_{\alpha\left(g_{1}\right)=g_{2}} \bigwedge_{m_{1} \in M_{1}}\left(\varphi \circ \varphi^{\vdash} \circ t \circ \beta\left(m_{1}\right) \longrightarrow I_{2}\left(\alpha\left(g_{1}\right), \beta\left(m_{1}\right)\right)\right) \\
& \stackrel{(\dagger)}{=} \bigvee_{\alpha\left(g_{1}\right)=g_{2}} \bigwedge_{m_{1} \in M_{1}}\left(t \circ \beta\left(m_{1}\right) \longrightarrow_{r} I_{2}\left(\alpha\left(g_{1}\right), \beta\left(m_{1}\right)\right)\right)
\end{aligned}
$$




$$
\begin{aligned}
& =\bigvee_{\alpha\left(g_{1}\right)=g_{2}} \bigwedge_{m_{1} \in M_{1}}\left(t \circ \beta\left(m_{1}\right) \longrightarrow_{r} I_{2}\left(g_{2}, \beta\left(m_{1}\right)\right)\right) \\
& \stackrel{(\dagger)}{=} \bigwedge_{m_{1} \in M_{1}}\left(t \circ \beta\left(m_{1}\right) \longrightarrow_{r} I_{2}\left(g_{2}, \beta\left(m_{1}\right)\right)\right) \\
& \stackrel{(\dagger)}{=} \bigwedge_{m_{2} \in M_{2}}\left(t\left(m_{2}\right) \longrightarrow_{r} I_{2}\left(g_{2}, m_{2}\right)\right) \\
& =\left(K_{2}(t)\right)\left(g_{2}\right),
\end{aligned}
$$

where $(\dagger)$ uses Definition 76, whereas ( $\dagger \dagger)$ uses the fact that $\mathscr{K}_{1} \stackrel{f}{\rightarrow} \mathscr{K}_{2}$ is a morphism of $\mathbf{S}-\mathbf{F C}_{m}^{G W}$.

5.2.3. From $\mathbf{S}-\mathbf{F C}{ }^{G W}$ to $\mathbf{S}-\mathbf{F C}_{a}^{D M R}$. In this subsection, we construct a functor from a particular subcategory of the category S-FC ${ }^{G W}$ to the category $\mathbf{S}-\mathbf{F C} \mathbf{C}_{a}^{D M R}$. As before, we start with singling out the subcategory in question.

Definition 78. S-FC $\mathbf{C}_{*}^{G W}$ is the (nonfull) subcategory of the category $\mathbf{S}-\mathbf{F C}{ }^{G W}$, with the same objects, whose morphisms $\mathscr{K}_{1} \stackrel{f}{\rightarrow} \mathscr{K}_{2}$ are such that the maps $G_{1} \stackrel{\alpha}{\rightarrow} G_{2}, M_{1} \stackrel{\beta}{\rightarrow} M_{2}$ are surjective, whereas the $\mathbf{S}^{o p}$-morphism $L_{2} \stackrel{\varphi^{o p}}{\longrightarrow} L_{1}$ is injective and, moreover, the map $\left|L_{1}\right| \stackrel{\varphi^{o p \vdash}}{\longrightarrow}\left|L_{2}\right|$ preserves $\bigvee, \rightarrow_{l}$ and $\rightarrow r$.

Theorem 79. There exists the functor $\mathbf{S}-\mathrm{FC}_{*}^{G W} \stackrel{H_{\mathrm{GDa}}}{\longrightarrow}$ S-FC ${ }_{a}^{D M R}$, which is defined by $H_{G D a}\left(\mathscr{K}_{1} \stackrel{f}{\rightarrow} \mathscr{K}_{2}\right)=$ $\mathscr{K}_{1} \stackrel{\left((\alpha, \varphi)^{\longmapsto,},(\beta, \varphi)^{\longmapsto)}\right)}{\longrightarrow} \mathscr{K}_{2}$.

Proof. To show that the functor is correct on morphisms, we verify commutativity of the diagrams

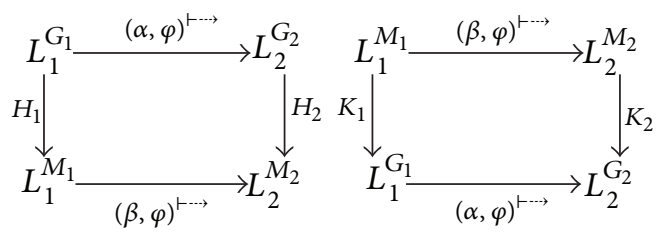

For the left-hand diagram, notice that given $s \in L_{1}^{G_{1}}$ and $m_{2} \in M_{2}$,

$$
\begin{aligned}
& \left((\beta, \varphi)^{\vdash \cdots} \circ H_{1}(s)\right)\left(m_{2}\right) \\
& \quad=\varphi^{o p \vdash}\left(\bigvee_{\beta\left(m_{1}\right)=m_{2}}\left(H_{1}(s)\right)\left(m_{1}\right)\right) \\
& \quad=\varphi^{o p \vdash}\left(\bigvee_{\beta\left(m_{1}\right)=m_{2}}\left(\bigwedge_{g_{1} \in G_{1}}\left(s\left(g_{1}\right) \longrightarrow{ }_{l} I_{1}\left(g_{1}, m_{1}\right)\right)\right)\right)
\end{aligned}
$$

$$
\begin{aligned}
& \stackrel{(\dagger)}{=} \varphi^{o p \vdash}\left(\bigvee _ { \beta ( m _ { 1 } ) = m _ { 2 } } \left(\bigwedge _ { g _ { 1 } \in G _ { 1 } } \left(s\left(g_{1}\right) \longrightarrow l \varphi^{o p}\right.\right.\right. \\
& \left.\left.\left.\quad \circ I_{2}\left(\alpha\left(g_{1}\right), \beta\left(m_{1}\right)\right)\right)\right)\right) \\
& =\varphi^{o p \vdash}\left(\bigvee _ { \beta ( m _ { 1 } ) = m _ { 2 } } \left(\bigwedge _ { g _ { 1 } \in G _ { 1 } } \left(s\left(g_{1}\right) \longrightarrow l \varphi^{o p}\right.\right.\right.
\end{aligned}
$$

$$
\begin{aligned}
& \left.\left.\left.\circ I_{2}\left(\alpha\left(g_{1}\right), m_{2}\right)\right)\right)\right) \\
& \stackrel{(\dagger \dagger)}{=} \varphi^{o p \vdash}\left(\bigwedge_{g_{1} \in G_{1}}\left(s\left(g_{1}\right) \longrightarrow{ }_{l} \varphi^{o p} \circ I_{2}\left(\alpha\left(g_{1}\right), m_{2}\right)\right)\right) \\
& \stackrel{(\dagger+)}{=} \bigwedge_{g_{1} \in G_{1}}\left(\varphi^{o p \vdash} \circ s\left(g_{1}\right) \longrightarrow l \varphi^{o p \vdash} \circ \varphi^{o p} \circ I_{2}\left(\alpha\left(g_{1}\right), m_{2}\right)\right) \\
& \stackrel{(\dagger \dagger)}{=} \bigwedge_{g_{1} \in G_{1}}\left(\varphi^{o p \vdash} \circ s\left(g_{1}\right) \longrightarrow I_{2}\left(\alpha\left(g_{1}\right), m_{2}\right)\right) \\
& =\bigwedge_{g_{2} \in G_{2}} \bigwedge_{\alpha\left(g_{1}\right)=g_{2}}\left(\varphi^{o p \vdash} \circ s\left(g_{1}\right) \longrightarrow_{l} I_{2}\left(g_{2}, m_{2}\right)\right) \\
& =\bigwedge_{g_{2} \in G_{2}}\left(\left(\bigvee_{\alpha\left(g_{1}\right)=g_{2}} \varphi^{o p \vdash} \circ s\left(g_{1}\right)\right) \longrightarrow_{l} I_{2}\left(g_{2}, m_{2}\right)\right) \\
& \stackrel{(\dagger \dagger)}{=} \bigwedge_{g_{2} \in G_{2}}\left(\varphi^{o p \vdash}\left(\bigvee_{\alpha\left(g_{1}\right)=g_{2}} s\left(g_{1}\right)\right) \longrightarrow_{l} I_{2}\left(g_{2}, m_{2}\right)\right) \\
& =\bigwedge_{g_{2} \in G_{2}}\left(\left((\alpha, \varphi)^{\vdash \cdots}(s)\right)\left(g_{2}\right) \longrightarrow I_{2}\left(g_{2}, m_{2}\right)\right) \\
& =\left(\left(H_{2} \circ(\alpha, \varphi)^{\vdash \cdots \mapsto}\right)(s)\right)\left(m_{2}\right) \text {, }
\end{aligned}
$$

where $(\dagger)$ uses the fact that $\mathscr{K}_{1} \stackrel{f}{\rightarrow} \mathscr{K}_{2}$ is a morphism of S-FC ${ }^{G W}$, whereas (††) employs Definition 78.

For the right-hand diagram, notice that given $t \in L_{1}^{M_{1}}$ and $g_{2} \in G_{2}$,

$$
\begin{aligned}
& \left(K_{2} \circ(\beta, \varphi)^{\vdash \cdots(}(t)\right)\left(g_{2}\right) \\
& \quad=\bigwedge_{m_{2} \in M_{2}}\left(\left((\beta, \varphi)^{\vdash \cdots(}(t)\right)\left(m_{2}\right) \longrightarrow_{r} I_{2}\left(g_{2}, m_{2}\right)\right) \\
& \quad=\bigwedge_{m_{2} \in M_{2}}\left(\varphi^{o p \vdash}\left(\underset{\beta\left(m_{1}\right)=m_{2}}{\bigvee} t\left(m_{1}\right)\right) \longrightarrow_{r} I_{2}\left(g_{2}, m_{2}\right)\right)
\end{aligned}
$$




$$
\begin{aligned}
& \stackrel{(\dagger)}{=} \bigwedge_{m_{2} \in M_{2}}\left(\left(\bigvee_{\beta\left(m_{1}\right)=m_{2}} \varphi^{o{ }^{\vdash}} \circ t\left(m_{1}\right) \longrightarrow_{r} I_{2}\left(g_{2}, m_{2}\right)\right)\right) \\
& =\bigwedge_{m_{2} \in M_{2}} \bigwedge_{\beta\left(m_{1}\right)=m_{2}}\left(\varphi^{o p^{\vdash}} \circ t\left(m_{1}\right) \longrightarrow{ }_{r} I_{2}\left(g_{2}, m_{2}\right)\right) \\
& =\bigwedge_{m_{1} \in M_{1}}\left(\varphi^{o p \vdash} \circ t\left(m_{1}\right) \longrightarrow_{r} I_{2}\left(g_{2}, \beta\left(m_{1}\right)\right)\right) \\
& \stackrel{(\dagger)}{=} \bigwedge_{m_{1} \in M_{1}}\left(\varphi^{o p \vdash} \circ t\left(m_{1}\right) \longrightarrow_{r} \varphi^{o p \vdash} \circ \varphi^{o p} \circ I_{2}\left(g_{2}, \beta\left(m_{1}\right)\right)\right) \\
& \stackrel{(\dagger)}{=} \varphi^{o p \vdash}\left(\bigwedge_{m_{1} \in M_{1}}\left(t\left(m_{1}\right) \longrightarrow_{r} \varphi^{o p} \circ I_{2}\left(g_{2}, \beta\left(m_{1}\right)\right)\right)\right) \\
& \stackrel{(\dagger)}{=} \varphi^{o p}\left(\bigvee _ { \alpha ( g _ { 1 } ) = g _ { 2 } } \left(\bigwedge _ { m _ { 1 } \in M _ { 1 } } \left(t\left(m_{1}\right) \longrightarrow_{r} \varphi^{o p}\right.\right.\right. \\
& \left.\left.\left.\circ I_{2}\left(g_{2}, \beta\left(m_{1}\right)\right)\right)\right)\right) \\
& =\varphi^{o p^{\vdash}}\left(\bigvee _ { \alpha ( g _ { 1 } ) = g _ { 2 } } \left(\bigwedge _ { m _ { 1 } \in M _ { 1 } } \left(t\left(m_{1}\right) \longrightarrow_{r} \varphi^{o p}\right.\right.\right. \\
& \left.\left.\left.\circ I_{2}\left(\alpha\left(g_{1}\right), \beta\left(m_{1}\right)\right)\right)\right)\right) \\
& \stackrel{(\dagger \dagger)}{=} \varphi^{o p \vdash}\left(\bigvee_{\alpha\left(g_{1}\right)=g_{2}}\left(\bigwedge_{m_{1} \in M_{1}}\left(t\left(m_{1}\right) \longrightarrow_{r} I_{1}\left(g_{1}, m_{1}\right)\right)\right)\right) \\
& =\varphi^{o p^{\vdash}}\left(\bigvee_{\alpha\left(g_{1}\right)=g_{2}}\left(K_{1}(t)\right)\left(g_{1}\right)\right) \\
& =\left((\alpha, \varphi)^{\vdash \cdots} \circ K_{1}(t)\right)\left(g_{2}\right) \text {, }
\end{aligned}
$$

where $(\dagger)$ uses Definition 78, whereas $(\dagger \dagger)$ employs the fact that $\mathscr{K}_{1} \stackrel{f}{\rightarrow} \mathscr{K}_{2}$ is a morphism of $\mathbf{S}-\mathbf{F C}{ }^{G W}$.

5.2.4. From $\mathbf{S}-\mathbf{F C} \mathbf{C}^{G W}$ to $\mathbf{S}-\mathbf{F} \mathbf{C}^{D M R}$. In this subsection, we construct a functor from a particular subcategory of the category $\mathbf{S}-\mathbf{F C}{ }^{G W}$ to the category $\mathbf{S}-\mathbf{F C}^{D M R}$. As before, we begin with singling out the subcategory in question.

Definition 80. $\mathbf{S}-\mathbf{F C}_{*}^{G W}$ is the (nonfull) subcategory of the category $\mathbf{S}$-FC ${ }^{G W}$, with the same objects, whose morphisms $\mathscr{K}_{1} \stackrel{f}{\rightarrow} \mathscr{K}_{2}$ are such that the maps $G_{1} \stackrel{\alpha}{\rightarrow} G_{2}, M_{1} \stackrel{\beta}{\rightarrow} M_{2}$ are surjective, whereas the $\boldsymbol{S}^{o p}$-morphism $L_{2} \stackrel{\varphi^{o p}}{\longrightarrow} L_{1}$ is an isomorphism.
Theorem 81. There exists the functor $\mathbf{S}-\mathbf{F C}_{*}^{G W} \stackrel{H_{G D}}{\longrightarrow}$ S-FC ${ }^{D M R}$, which is defined by $H_{G D}\left(\mathscr{K}_{1} \stackrel{f}{\rightarrow} \mathscr{K}_{2}\right)=$ $\mathscr{K}_{1} \stackrel{\left((\alpha, \varphi)^{\vdash \cdots},\left((\beta, \varphi)^{\leftarrow}\right)^{o p}\right)}{\longrightarrow} \mathscr{K}_{2}$.

Proof. To show that the functor is correct on morphisms, we verify commutativity of the diagrams

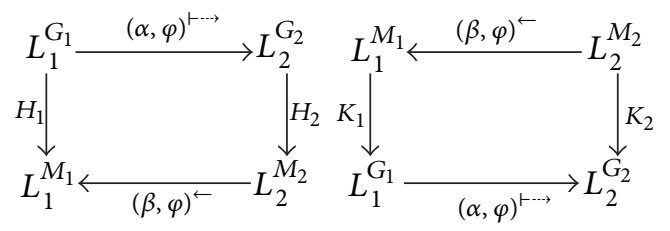

For the left-hand diagram, notice that given $s \in L_{1}^{G_{1}}$ and $m_{1} \in M_{1}$,

$$
\begin{aligned}
& \left((\beta, \varphi)^{\leftarrow} \circ H_{2} \circ(\alpha, \varphi)^{\vdash \cdots \rightarrow}(s)\right)\left(m_{1}\right) \\
& =\varphi^{o p} \circ\left(H_{2}\left((\alpha, \varphi)^{\vdash \cdots \cdots}(s)\right)\right) \circ \beta\left(m_{1}\right) \\
& =\varphi^{o p}\left(\bigwedge_{g_{2} \in G_{2}}\left(\left((\alpha, \varphi)^{\vdash \cdots \cdots}(s)\right)\left(g_{2}\right) \longrightarrow_{l} I_{2}\left(g_{2}, \beta\left(m_{1}\right)\right)\right)\right) \\
& =\varphi^{o p}\left(\bigwedge _ { g _ { 2 } \in G _ { 2 } } \left(\varphi^{o p}\left(\bigvee_{\alpha\left(g_{1}\right)=g_{2}} s\left(g_{1}\right)\right)\right.\right. \\
& \left.\longrightarrow{ }_{l} I_{2}\left(g_{2}, \beta\left(m_{1}\right)\right)\right) \\
& \stackrel{(\dagger)}{=} \varphi^{o p}\left(\bigwedge _ { g _ { 2 } \in G _ { 2 } } \left(\bigwedge _ { \alpha ( g _ { 1 } ) = g _ { 2 } } \left(\varphi^{o p^{\vdash}} \circ s\left(g_{1}\right)\right.\right.\right. \\
& \left.\left.\longrightarrow_{l} I_{2}\left(g_{2}, \beta\left(m_{1}\right)\right)\right)\right) \\
& =\varphi^{o p}\left(\bigwedge_{g_{1} \in G_{1}}\left(\varphi^{o p \vdash} \circ s\left(g_{1}\right) \longrightarrow_{l} I_{2}\left(\alpha\left(g_{1}\right), \beta\left(m_{1}\right)\right)\right)\right) \\
& \stackrel{(\dagger)}{=} \bigwedge_{g_{1} \in G_{1}}\left(\varphi^{o p} \circ \varphi^{o p \vdash} \circ s\left(g_{1}\right) \longrightarrow{ }_{l} \varphi^{o p} \circ I_{2}\left(\alpha\left(g_{1}\right), \beta\left(m_{1}\right)\right)\right) \\
& \stackrel{(\dagger)}{=} \bigwedge_{g_{1} \in G_{1}}\left(s\left(g_{1}\right) \longrightarrow_{l} \varphi^{o p} \circ I_{2}\left(\alpha\left(g_{1}\right), \beta\left(m_{1}\right)\right)\right) \\
& \stackrel{(\dagger)}{=} \bigwedge_{g_{1} \in G_{1}}\left(s\left(g_{1}\right) \longrightarrow{ }_{l} I_{1}\left(g_{1}, m_{1}\right)\right)=\left(H_{1}(s)\right)\left(m_{1}\right) \text {, }
\end{aligned}
$$

where $(\dagger)$ uses Definition 80, whereas $(\dagger \dagger)$ employs the fact that $\mathscr{K}_{1} \stackrel{f}{\rightarrow} \mathscr{K}_{2}$ is a morphism of $\mathbf{S}-\mathbf{F C}{ }^{G W}$. 
For the right-hand diagram, notice that given $t \in L_{2}^{M_{2}}$ and $g_{2} \in G_{2}$,

$$
\begin{aligned}
& \left((\alpha, \varphi)^{\vdash \cdots,} \circ K_{1} \circ(\beta, \varphi)^{\leftarrow}(t)\right)\left(g_{2}\right) \\
& =\varphi^{o p \vdash}\left(\bigvee_{\alpha\left(g_{1}\right)=g_{2}}\left(K_{1} \circ(\beta, \varphi)^{\leftarrow}(t)\right)\left(g_{1}\right)\right) \\
& =\varphi^{o p \vdash}\left(\bigvee _ { \alpha ( g _ { 1 } ) = g _ { 2 } } \left(\bigwedge _ { m _ { 1 } \in M _ { 1 } } \left(\left((\beta, \varphi)^{\leftarrow}(t)\right)\left(m_{1}\right)\right.\right.\right. \\
& \left.\left.\left.\longrightarrow_{r} I_{1}\left(g_{1}, m_{1}\right)\right)\right)\right) \\
& =\varphi^{o p \vdash}\left(\bigvee _ { \alpha ( g _ { 1 } ) = g _ { 2 } } \left(\bigwedge _ { m _ { 1 } \in M _ { 1 } } \left(\varphi^{o p} \circ t \circ \beta\left(m_{1}\right)\right.\right.\right. \\
& \left.\left.\left.\longrightarrow_{r} I_{1}\left(g_{1}, m_{1}\right)\right)\right)\right) \\
& \stackrel{(\dagger)}{=} \varphi^{o p \vdash}\left(\bigvee _ { \alpha ( g _ { 1 } ) = g _ { 2 } } \left(\bigwedge _ { m _ { 1 } \in M _ { 1 } } \left(\varphi^{o p} \circ t \circ \beta\left(m_{1}\right) \longrightarrow_{r} \varphi^{o p}\right.\right.\right. \\
& \left.\left.\left.\circ I_{2}\left(\alpha\left(g_{1}\right), \beta\left(m_{1}\right)\right)\right)\right)\right) \\
& =\varphi^{o p \vdash}\left(\bigvee _ { \alpha ( g _ { 1 } ) = g _ { 2 } } \left(\bigwedge _ { m _ { 1 } \in M _ { 1 } } \left(\varphi^{o p} \circ t \circ \beta\left(m_{1}\right) \longrightarrow_{r} \varphi^{o p}\right.\right.\right. \\
& \left.\left.\circ I_{2}\left(g_{2}, \beta\left(m_{1}\right)\right)\right)\right) \\
& \stackrel{(+\dagger)}{=} \varphi^{o p \vdash}\left(\bigwedge _ { m _ { 1 } \in M _ { 1 } } \left(\varphi^{o p} \circ t \circ \beta\left(m_{1}\right) \longrightarrow_{r} \varphi^{o p}\right.\right. \\
& \left.\left.\circ I_{2}\left(g_{2}, \beta\left(m_{1}\right)\right)\right)\right) \\
& \stackrel{(+\dagger)}{=} \varphi^{o p \vdash}\left(\bigwedge_{m_{2} \in M_{2}}\left(\varphi^{o p} \circ t\left(m_{2}\right) \longrightarrow_{r} \varphi^{o p} \circ I_{2}\left(g_{2}, m_{2}\right)\right)\right) \\
& \stackrel{(\dagger \dagger)}{=} \bigwedge_{m_{2} \in M_{2}}\left(\varphi^{o p \vdash} \circ \varphi^{o p} \circ t\left(m_{2}\right) \longrightarrow_{r} \varphi^{o p \vdash} \circ \varphi^{o p} \circ I_{2}\left(g_{2}, m_{2}\right)\right) \\
& \stackrel{(\dagger \dagger)}{=} \bigwedge_{m_{2} \in M_{2}}\left(t\left(m_{2}\right) \longrightarrow{ }_{r} I_{2}\left(g_{2}, m_{2}\right)\right)=\left(K_{2}(t)\right)\left(g_{2}\right),
\end{aligned}
$$

where $(\dagger)$ uses the fact that $\mathscr{K}_{1} \stackrel{f}{\rightarrow} \mathscr{K}_{2}$ is a morphism of S-FC ${ }^{G W}$, whereas ( ††) employs Definition 80.
5.2.5. From $\mathbf{S}-\mathbf{F C}_{a}^{D M R}$ to $\mathbf{S}-\mathbf{F C} \mathbf{C}_{m}^{G W}$. In this subsection, we construct functorial embeddings of several subcategories of the category $\mathbf{S}-\mathbf{F C}_{a}^{D M R}$ into the category $\mathbf{S}-\mathbf{F C} \mathbf{C}_{m}^{G W}$. We begin with singling out the first subcategory in question. The reader should notice that similar to Section 5.1.5, all the subcategories constructed in the current one are fixed-basis.

Definition 82. Given an $\mathbf{L}$-algebra $L, L-\mathbf{F C}_{a \mathrm{i}}^{D M R}$ is the (nonfull) subcategory of the category $L-\mathbf{F C}_{a}^{D M R}$, with the same objects, whose morphisms $\mathscr{K}_{1} \stackrel{f}{\rightarrow} \mathscr{K}_{2}$ have the property that the maps $L^{G_{1}} \stackrel{\alpha}{\rightarrow} L^{G_{2}}, L^{M_{1}} \stackrel{\beta}{\rightarrow} L^{M_{2}}$ are injective.

Theorem 83. There exists the functor $L-\mathbf{F C}_{a i}^{D M R} \stackrel{H_{D a G m}^{i}}{\longrightarrow}$ S-FC ${ }_{m}^{G W}$, which is defined by the formula $H_{D a G m}^{\mathrm{i}}\left(\mathscr{K}_{1} \stackrel{f}{\rightarrow}\right.$ $\left.\mathscr{K}_{2}\right)=\left(L^{G_{1}}, L^{M_{1}}, L, \widehat{I}_{1}\right) \stackrel{\left(\alpha, \beta, 1_{L}\right)}{\longrightarrow}\left(L^{G_{2}}, L^{M_{2}}, L, \widehat{I}_{2}\right)$, where

$$
\widehat{I}_{j}(s, t)= \begin{cases}T_{L}, & (s, t) \text { is a formal concept of } \mathscr{K}_{j} \\ \perp_{L}, & \text { otherwise. }\end{cases}
$$

Proof. We have to show that the functor is correct on morphisms. Given $s_{1} \in L^{G_{1}}$ and $t_{1} \in L^{M_{1}}$, it follows that $\widehat{I}_{1}\left(s_{1}, t_{1}\right)=\top_{L}$ iff $\left(s_{1}, t_{1}\right)$ is a formal concept of $\mathscr{K}_{1}$ iff $\left(\alpha\left(s_{1}\right), \beta\left(t_{1}\right)\right)$ is a formal concept of $\mathscr{K}_{2}$ iff $\widehat{I}_{2}\left(\alpha\left(s_{1}\right), \beta\left(t_{1}\right)\right)=$ $\mathrm{T}_{L}$, where the second "iff" uses Theorem 46(1).

To make the functor into an embedding, one uses the machinery of Theorem 64 .

Theorem 84. For every qsrs-algebra $L$, the functor $L-\mathbf{F C}_{a \mathrm{i}}^{D M R} \stackrel{H_{D a G m}^{\mathrm{i}}}{\longrightarrow} \mathbf{S}_{-} \mathbf{F C}_{m}^{G W}$ is a nonfull embedding.

Proof. Follow the steps of the proof of Theorem 64.

To continue, we provide the second of the claimed embeddings. We begin with singling out the subcategory of the category $\mathbf{S}-\mathbf{F C} C_{a}^{D M R}$, which is suitable for the occasion.

Definition 85. Given an L-algebra L, L-FC $C_{a i \alpha}^{D M R}$ (resp., $L-\mathrm{FC}_{a i \beta}^{D M R}$ ) is the (nonfull) subcategory of the category $L$-FC ${ }_{a}^{D M R}$, with the same objects, whose morphisms $\mathscr{K}_{1} \stackrel{f}{\rightarrow}$ $\mathscr{K}_{2}$ have the property that the map $L^{G_{1}} \stackrel{\alpha}{\rightarrow} L^{G_{2}}$ (resp., $L^{M_{1}} \stackrel{\beta}{\rightarrow}$ $\left.L^{M_{2}}\right)$ is injective.

Theorem 86. Let $\dagger$ be either $\alpha$ or $\beta$. There exists the functor $L-\mathbf{F C}_{a i \dagger}^{D M R} \stackrel{H_{D a G m}^{i \dagger}}{\longrightarrow} \mathbf{S}_{-} \mathbf{F C}_{m}^{G W}$, which is given by $H_{D a G m}^{i \dagger}\left(\mathscr{K}_{1} \stackrel{f}{\rightarrow}\right.$ $\left.\mathscr{K}_{2}\right)=\left(L^{G_{1}}, L^{M_{1}}, L, \widehat{I}_{1}\right) \stackrel{\left(\alpha, \beta, 1_{L}\right)}{\longrightarrow}\left(L^{G_{2}}, L^{M_{2}}, L, \widehat{I}_{2}\right)$, where

$$
\widehat{I}_{j}(s, t)= \begin{cases}T_{L}, & (s, t) \text { is a formal protoconcept of } \mathscr{K}_{j} \\ \perp_{L}, & \text { otherwise. }\end{cases}
$$

Proof. We have to show that the functor is correct on morphisms. Given $s_{1} \in L^{G_{1}}$ and $t_{1} \in L^{M_{1}}$, it follows that $\widehat{I}_{1}\left(s_{1}, t_{1}\right)=\mathrm{T}_{L}$ iff $\left(s_{1}, t_{1}\right)$ is a formal protoconcept 
of $\mathscr{K}_{1}$ iff $\left(\alpha\left(s_{1}\right), \beta\left(t_{1}\right)\right)$ is a formal protoconcept of $\mathscr{K}_{2}$ iff $\widehat{I}_{2}\left(\alpha\left(s_{1}\right), \beta\left(t_{1}\right)\right)=\mathrm{T}_{L}$, where the second "iff" uses Theorem 46(2).

Theorem 87. Let $\dagger$ be either $\alpha$ or $\beta$. For every qsrs-algebra $L$, the functor $L-\mathbf{F C}_{a i \dagger}^{D M R} \stackrel{H_{D a G m}^{i \dagger}}{\longrightarrow} \mathbf{S}-\mathbf{F C}_{m}^{G W}$ is a nonfull embedding.

Proof. Follow the steps of the proof of Theorem 64.

To continue, we show the last one of the claimed embeddings.

Definition 88. Given an L-algebra L, L-FC $\mathbf{C}_{\text {aoed }}^{D M R}$ (resp., $L-F_{\text {aoe } \beta}^{D M R}$ ) is the (nonfull) subcategory of the category $L-$ FC $_{a}^{D M R}$, with the same objects, whose morphisms $\mathscr{K}_{1} \stackrel{f}{\rightarrow}$ $\mathscr{K}_{2}$ have the property that the map $L^{G_{1}} \stackrel{\alpha}{\rightarrow} L^{G_{2}}$ (resp., $L^{M_{1}} \stackrel{\beta}{\rightarrow}$ $L^{M_{2}}$ ) is an order embedding (i.e., both order-preserving and order-reflecting).

Theorem 89. Let $\dagger$ be either $\alpha$ or $\beta$. There exists the functor $L-\mathbf{F C}_{\text {aoe }}^{D M R} \stackrel{H_{D a G m}^{o e \dagger}}{\longrightarrow} \mathbf{S}_{-} \mathbf{F C}_{m}^{G W}$, which is given by $H_{D a G m}^{o e \dagger}\left(\mathscr{K}_{1} \stackrel{f}{\rightarrow}\right.$ $\left.\mathscr{K}_{2}\right)=\left(L^{G_{1}}, L^{M_{1}}, L, \widehat{I}_{1}\right) \stackrel{\left(\alpha, \beta, 1_{L}\right)}{\longrightarrow}\left(L^{G_{2}}, L^{M_{2}}, L, \widehat{I}_{2}\right)$, where

$$
\widehat{I}_{j}(s, t)= \begin{cases}\top_{L}, & (s, t) \text { is a formal preconcept of } \mathscr{K}_{j} \\ \perp_{L}, & \text { otherwise. }\end{cases}
$$

Proof. We have to show that the functor is correct on morphisms. Given $s_{1} \in L^{G_{1}}$ and $t_{1} \in L^{M_{1}}$, it follows that $\widehat{I}_{1}\left(s_{1}, t_{1}\right)=\mathrm{T}_{L}$ iff $\left(\mathrm{s}_{1}, t_{1}\right)$ is a formal preconcept of $\mathscr{K}_{1}$ iff $\left(\alpha\left(s_{1}\right), \beta\left(t_{1}\right)\right)$ is a formal preconcept of $\mathscr{K}_{2}$ iff $\widehat{I}_{2}\left(\alpha\left(s_{1}\right), \beta\left(t_{1}\right)\right)=\mathrm{T}_{L}$, where the second "iff" uses Theorem 46(3).

Theorem 90. Let $\dagger$ be either $\alpha$ or $\beta$. For every qsrs-algebra $L$, the functor $L-\mathbf{F C}_{a o e \dagger}^{D M R} \stackrel{H_{D a G m}^{o e \dagger}}{\longrightarrow} \mathbf{S}-\mathbf{F C} C_{m}^{G W}$ is a nonfull embedding.

Proof. Follow the steps of the proof of Theorem 64.

As a last remark, we notice that the case of $\mathbf{L}$ extending the variety UQuant simplifies the previously mentioned results (see the remark at the end of Section 5.1.5).

5.3. $\mathbf{S}-\mathbf{F C}{ }^{G W}$ and $\mathbf{S}-\mathbf{F C}_{m}^{G W}$ versus $\mathbf{S}-\mathbf{F C}{ }^{C}$ and $\mathbf{S}-\mathbf{F C}_{m}^{C}$. In this subsection, we consider possible functorial links between the categories $\mathbf{S}-\mathbf{F C} \mathbf{C}^{G W}$ and $\mathbf{S}-\mathbf{F} \mathbf{C}_{m}^{G W}$, on one side, and the categories $\mathbf{S}-\mathbf{F C}$ and $\mathbf{S}-\mathbf{F} C_{m}^{C}$, on the other.

5.3.1. From $\mathbf{S}-\mathbf{F C}{ }_{m}^{G W}$ to $\mathbf{S}-\mathbf{F} \mathbf{C}_{m}^{C}$. In this subsection, we construct a functorial isomorphism between particular subcategories of the categories $\mathbf{S}-\mathbf{F C}_{m}^{G W}$ and $\mathbf{S}-\mathbf{F C}_{m}^{C}$, respectively.

Definition 91. S-FC $\mathbf{m *}^{G W}$ (resp., $\mathbf{S}-\mathbf{F C}_{m *}^{\mathrm{C}}$ ) is the (nonfull) subcategory of the category $\mathbf{S}-\mathbf{F C}_{m}^{G W}$ (resp., $\mathbf{S}-\mathbf{F} \mathbf{C}_{m}^{C}$ ), with the same objects, whose morphisms $\mathscr{K}_{1} \stackrel{f}{\rightarrow} \mathscr{K}_{2}$ have the property that the map $M_{1} \stackrel{\beta}{\rightarrow} M_{2}$ (resp., $M_{2} \stackrel{\beta^{o p}}{\rightarrow} M_{1}$ ) is bijective.

The categories of Definition 91 give rise to the following functors.

Theorem 92. There exist two functors

(1) $\mathbf{S}-\mathbf{F C} C_{m *}^{G W} \stackrel{H_{G m C m}}{\longrightarrow} \quad \mathbf{S}-\mathbf{F C}_{m *}^{C}$, which is given by $H_{\mathrm{GmCm}}\left(\mathscr{K}_{1} \stackrel{f}{\rightarrow} \mathscr{K}_{2}\right)=\mathscr{K}_{1} \stackrel{\left(\alpha,\left(\beta^{-1}\right)^{o p}, \varphi\right)}{\longrightarrow} \mathscr{K}_{2}$,

(2) $\mathbf{S}-\mathbf{F} C_{m *}^{C} \stackrel{H_{C m G m}}{\longrightarrow} \mathbf{S}-\mathbf{F C}_{m *}^{G W}$, which is given by $H_{\mathrm{CmGm}}\left(\mathscr{K}_{1} \stackrel{f}{\rightarrow} \mathscr{K}_{2}\right)=\mathscr{K}_{1} \stackrel{\left(\alpha,\left(\beta^{o p}\right)^{-1}, \varphi\right)}{\longrightarrow} \mathscr{K}_{2}$,

for which $H_{\mathrm{GmCm}} \circ H_{\mathrm{CmGm}}=1_{\mathbf{S}-\mathbf{F C}_{m *}^{C}}$ and $H_{\mathrm{CmGm}} \circ H_{\mathrm{GmCm}}=$ $1_{\mathbf{S}-\mathbf{F C}_{m *}^{G W}}$.

Proof. To show that the functor $H_{G m C m}$ is correct on morphisms, notice that given $g_{1} \in G_{1}$ and $m_{2} \in M_{2}$, it follows that $\varphi \circ I_{1}\left(g_{1}, \beta^{-1}\left(m_{2}\right)\right)=I_{2}\left(\alpha\left(g_{1}\right), \beta \circ \beta^{-1}\left(m_{2}\right)\right)=I_{2}\left(\alpha\left(g_{1}\right), m_{2}\right)$. Additionally, to show that the functor $H_{\mathrm{CmGm}}$ is correct on morphisms, notice that given $g_{1} \in G_{1}$ and $m_{1} \in M_{1}$, it follows that $\varphi \circ I_{1}\left(g_{1}, m_{1}\right)=\varphi \circ I_{1}\left(g_{1}, \beta^{o p}\left(m_{2}\right)\right)=I_{2}\left(\alpha\left(g_{1}\right), m_{2}\right)=$ $I_{2}\left(\alpha\left(g_{1}\right),\left(\beta^{o p}\right)^{-1}\left(m_{1}\right)\right)$.

5.3.2. From $\mathbf{S}-\mathbf{F C} \mathbf{C}^{G W}$ to $\mathbf{S}-\mathbf{F C}$. In this subsection, we construct an isomorphism between particular subcategories of the categories $\mathbf{S}-\mathbf{F C}{ }^{G W}$ and $\mathbf{S}-\mathbf{F C}{ }^{C}$, respectively.

Definition 93. $\mathbf{S}-\mathbf{F C}_{*}^{G W}$ (resp., $\mathbf{S}-\mathbf{F C}_{*}^{C}$ ) is the (nonfull) subcategory of the category $\mathbf{S}-\mathbf{F C}{ }^{G W}$ (resp., $\mathbf{S}-\mathbf{F C}^{C}$ ), with the same objects, whose morphisms $\mathscr{K}_{1} \stackrel{f}{\rightarrow} \mathscr{K}_{2}$ have the property that the $\operatorname{map} M_{1} \stackrel{\beta}{\rightarrow} M_{2}$ (resp., $M_{2} \stackrel{\beta^{o p}}{\longrightarrow} M_{1}$ ) is bijective.

The categories of Definition 93 give rise to the following functors.

Theorem 94. There exist two functors

(1) $\mathbf{S}-\mathbf{F C}_{*}^{G W} \stackrel{H_{\mathrm{GC}}}{\longrightarrow} \mathbf{S}-\mathbf{F C}_{*}^{C}$, which is given by $H_{\mathrm{GC}}\left(\mathscr{K}_{1} \stackrel{f}{\rightarrow}\right.$ $\left.\mathscr{K}_{2}\right)=\mathscr{K}_{1} \stackrel{\left(\alpha,\left(\beta^{-1}\right)^{o p}, \varphi\right)}{\longrightarrow} \mathscr{K}_{2}$,

(2) $\mathbf{S}-\mathbf{F C}_{*}^{C} \stackrel{H_{C G}}{\longrightarrow} \mathbf{S}_{*} \mathbf{F C}_{*}^{G W}$, which is given by $H_{C G}\left(\mathscr{K}_{1} \stackrel{f}{\rightarrow}\right.$ $\left.\mathscr{K}_{2}\right)=\mathscr{K}_{1} \stackrel{\left(\alpha,\left(\beta^{o p}\right)^{-1}, \varphi\right)}{\longrightarrow} \mathscr{K}_{2}$,

for which $H_{\mathrm{GC}} \circ H_{\mathrm{CG}}=1_{\mathbf{S}-\mathbf{F C}_{*}^{C}}$ and $H_{\mathrm{CG}} \circ H_{\mathrm{GC}}=1_{\mathbf{S}-\mathbf{F C}_{*}^{G W}}$.

Proof. To show that the functor $H_{G C}$ is correct on morphisms, notice that given $g_{1} \in G_{1}$ and $m_{2} \in M_{2}$, it follows that $I_{1}\left(g_{1}, \beta^{-1}\left(m_{2}\right)\right)=\varphi^{o p} \circ I_{2}\left(\alpha\left(g_{1}\right), \beta \circ \beta^{-1}\left(m_{2}\right)\right)=\varphi^{o p} \circ$ $I_{2}\left(\alpha\left(g_{1}\right), m_{2}\right)$. Additionally, to show that the functor $H_{C G}$ is correct on morphisms, notice that given $g_{1} \in G_{1}$ and $m_{1} \in M_{1}$, it follows that $I_{1}\left(g_{1}, m_{1}\right)=I_{1}\left(g_{1}, \beta^{o p}\left(m_{2}\right)\right)=$ $\varphi^{o p} \circ I_{2}\left(\alpha\left(g_{1}\right), m_{2}\right)=I_{2}\left(\alpha\left(g_{1}\right),\left(\beta^{o p}\right)^{-1}\left(m_{1}\right)\right)$. 
5.4. $L-F C^{C}$ versus $L-F C^{D M R}$. In this subsection, we make our framework closer to that of Denniston et al. [29], that is, consider a fixed-basis approach to lattice-valued FCA. We construct several functors between the categories $L-$ FC $^{C}$ and $L-F^{D M R}$ and show that they fail to provide an adjoint situation between the categories in question.

5.4.1. From $L-F C^{C}$ to $L-F C^{D M R}$. This subsection provides a functor from the category $L-F_{C} C$ to the category $L-F C^{D M R}$. More precisely, Theorems 52 and 53 (or, also, Theorems 59 and 60 ) give rise to the following result.

Definition 95. Given an L-algebra $L, L-\mathbf{F C}_{*}^{C}$ is the (nonfull) subcategory of the category $L-F_{C}{ }^{C}$, with the same objects and with morphisms $\mathscr{K}_{1} \stackrel{f}{\rightarrow} \mathscr{K}_{2}$ such that the maps $G_{1} \stackrel{\alpha}{\rightarrow} G_{2}$, $M_{2} \stackrel{\beta^{o p}}{\longrightarrow} M_{1}$ are surjective.

Theorem 96. There exists the functor $L-\mathbf{F C}_{*}^{C} \stackrel{H_{L C L D}}{\longrightarrow}$ $L-F_{C}^{D M R}$, which is given by $H_{L C L D}\left(\mathscr{K}_{1} \stackrel{f}{\rightarrow} \mathscr{K}_{2}\right)=$ $\mathscr{K}_{1} \stackrel{\left(\alpha_{L},\left(\left(\beta^{o p}\right)_{L}\right)^{o p}\right)}{\longrightarrow} \mathscr{K}_{2}$. If $\top_{L} \neq \perp_{L}$, then $H_{L C L D}$ is a nonfull embedding.

Proof. It is enough to show that the functor is faithful (we do not require $L$ to have the unit).

Given some $L$-FC ${ }^{C}$-morphisms $\mathscr{K}_{1} \underset{f_{2}}{\stackrel{f_{1}}{\rightrightarrows}} \mathscr{K}_{2}$ such that $H_{L C L D}\left(f_{1}\right)=H_{L C L D}\left(f_{2}\right)$, it follows that $\left(\alpha_{1 L} \vec{L},\left(\left(\beta_{1}^{o p}\right)_{L} \rightarrow\right)^{o p}\right)=$ $\left(\alpha_{2 L} \vec{L},\left(\left(\beta_{2}^{o p}\right)_{L}\right)^{o p}\right)$ and, therefore, $\alpha_{1 L} \vec{b}=\alpha_{2 L} \rightarrow,\left(\beta_{1}^{o p}\right)_{L} \overrightarrow{=}$ $\left(\beta_{2}^{o p}\right)_{L} \rightarrow$. To show that $\alpha_{1}=\alpha_{2}$, notice that given $g_{1} \in G_{1}$,

$$
\begin{aligned}
\left(\alpha_{1 L} \rightarrow\left(\chi_{\left\{g_{1}\right\}}^{\top_{L}}\right)\right)\left(g_{2}\right) & =\bigvee_{\alpha_{1}\left(g_{1}^{\prime}\right)=g_{2}} \chi_{\left\{g_{1}\right\}}^{\top_{L}}\left(g_{1}^{\prime}\right) \\
& = \begin{cases}\top_{L}, & g_{2}=\alpha_{1}\left(g_{1}\right)=\chi_{\left\{\alpha_{1}\left(g_{1}\right)\right\}}^{\top_{L}}\left(g_{2}\right) \\
\perp_{L}, & \text { otherwise }\end{cases}
\end{aligned}
$$

for every $g_{2} \in G_{2}$. As a consequence, $\chi_{\left\{\alpha_{1}\left(g_{1}\right)\right\}}^{\top_{L}}=\alpha_{1 L} \rightarrow\left(\chi_{\left\{g_{1}\right\}}^{\top_{L}}\right)=$ $\alpha_{2 L} \vec{L}\left(\chi_{\left\{g_{1}\right\}}^{\top_{L}}\right)=\chi_{\left\{\alpha_{2}\left(g_{1}\right)\right\}}^{\top_{L}}$. The assumption $\mathrm{T}_{L} \neq \perp_{L}$ then gives $\alpha_{1}\left(g_{1}\right)=\alpha_{2}\left(g_{1}\right)$. Similar technique can be employed to show that $\beta_{1}^{o p}=\beta_{2}^{o p}$.

5.4.2. From $L-F_{C} C^{D M R}$ to $L-F C^{C}$. This subsection shows functorial embeddings of some subcategories of the category $L-F C^{D M R}$ into the category $L-F_{C} C$. The results of Section 5.1.5 (with their respective notations) suggest the following.

Theorem 97. Every qsrs-algebra $L$ provides the following nonfull embeddings.
(1) $L-\mathbf{F C}_{\mathfrak{i}}^{D M R} \stackrel{H_{L D L C}^{\mathrm{i}}}{\longrightarrow} L-\mathbf{F C}^{C}$ given by $H_{L D L C}^{\mathfrak{i}}\left(\mathscr{K}_{1} \stackrel{f}{\rightarrow}\right.$ $\left.\mathscr{K}_{2}\right)=\left(L^{G_{1}}, L^{M_{1}}, \widehat{I}_{1}\right) \stackrel{(\alpha, \beta)}{\longrightarrow}\left(L^{G_{2}}, L^{M_{2}}, \widehat{I}_{2}\right)$, where

$\widehat{I}_{j}(s, t)= \begin{cases}\top_{L}, & (s, t) \text { is a formal concept of } \mathscr{K}_{j} \\ \perp_{L}, & \text { otherwise. }\end{cases}$

(2) $L-\mathbf{F C}_{r f p}^{D M R} \stackrel{H_{L D L C}^{r f p}}{\longrightarrow} L-\mathbf{F C}^{C}$ given by $H_{L D L C}^{r f p}\left(\mathscr{K}_{1} \stackrel{f}{\rightarrow}\right.$ $\left.\mathscr{K}_{2}\right)=\left(L^{G_{1}}, L^{M_{1}}, \widehat{I}_{1}\right) \stackrel{(\alpha, \beta)}{\longrightarrow}\left(L^{G_{2}}, L^{M_{2}}, \widehat{I}_{2}\right)$, where

$\widehat{I}_{j}(s, t)= \begin{cases}\top_{L}, & (s, t) \text { is a formal concept of } \mathscr{K}_{j} \\ \perp_{L}, & \text { otherwise. }\end{cases}$

(3) $L-\mathbf{F C}_{\text {orp }}^{D M R} \stackrel{H_{L D L C}^{\text {orp }}}{\longrightarrow} L-\mathbf{F C}^{C}$ given by $H_{L D L C}^{\text {orp }}\left(\mathscr{K}_{1} \stackrel{f}{\rightarrow}\right.$ $\left.\mathscr{K}_{2}\right)=\left(L^{G_{1}}, L^{M_{1}}, \widehat{I}_{1}\right) \stackrel{(\alpha, \beta)}{\longrightarrow}\left(L^{G_{2}}, L^{M_{2}}, \widehat{I}_{2}\right)$, where

$\widehat{I}_{j}(s, t)= \begin{cases}\top_{L}, & (s, t) \text { is a formal preconcept of } \mathscr{K}_{j} \\ \perp_{L}, & \text { otherwise. }\end{cases}$

(4) $L-\mathbf{F C}^{D M R} \stackrel{H_{L D L C}}{\longrightarrow} L-\mathbf{F C}^{C}$ given by $H_{L D L C}\left(\mathscr{K}_{1} \stackrel{f}{\rightarrow}\right.$ $\left.\mathscr{K}_{2}\right)=\left(L^{G_{1}}, L^{M_{1}}, \widehat{I}_{1}\right) \stackrel{(\alpha, \beta)}{\longrightarrow}\left(L^{G_{2}}, L^{M_{2}}, \widehat{I}_{2}\right)$, where

$\widehat{I}_{j}(s, t)= \begin{cases}\top_{L}, & (s, t) \text { is a formal protoconcept of } \mathscr{K}_{j} \\ \perp_{L}, & \text { otherwise. }\end{cases}$

Proof. The only thing that requires verification is nonfullness of the embeddings. We provide the explicit proof for the last item, which can be easily adapted for each of the remaining ones.

Consider the crisp case $\mathbf{L}=$ CBAlg, $L=2$, and let $G=M=|L|, I=G \times M$. We define (cf. Lemma 43) the $L$-FC ${ }^{D M R}$-object $\mathscr{K}=(G, M, I)$ where $I=\top_{L}$, and thus, $H=\mathrm{\top}_{L}$ and $K=\mathrm{\top}_{L}$. For every $S \in \mathscr{P}(\overline{G)}$ and every $T \in \overline{\overline{\mathscr{P}}(M)}, H \circ K(\overline{\overline{T)}}=M=H(S)$ and thus, $(S, T)$ is a formal protoconcept of $\mathscr{K}$. Thus, $\widehat{I}=\mathrm{\top}_{L}$, and, therefore, every pair of maps $\mathscr{P}(G) \stackrel{\alpha}{\rightarrow} \mathscr{P}(G), \mathscr{P}(M) \stackrel{\overrightarrow{\beta^{o p}}}{\longrightarrow} \mathscr{P}(M)$ is then an $L$-FC ${ }^{C}$-morphism $\left(H_{L D L C}(\mathscr{K})=(\mathscr{P}(G), \mathscr{P}(M), \widehat{I})\right) \stackrel{(\alpha, \beta)}{\longrightarrow}$ $H_{L D L C}(\mathscr{K})$. Define now $\mathscr{P}(G) \stackrel{\alpha}{\rightarrow} \mathscr{P}(G)=\mathscr{P}(G) \stackrel{1_{\mathscr{P}(G)}}{\longrightarrow}$ $\mathscr{P}(G), \mathscr{P}(M) \stackrel{\beta^{o p}}{\longrightarrow} \mathscr{P}(M)=\mathscr{P}(M) \stackrel{\emptyset}{\rightarrow} \mathscr{P}(M)$, and get an $L$-FC ${ }^{C}$-morphism $H_{L D L C}(\mathscr{K}) \stackrel{(\alpha, \beta)}{\longrightarrow} H_{L D L C}(\mathscr{K})$. However, $\beta^{o p} \circ H \circ \alpha(G)=\beta^{o p} \circ H(G)=\beta^{o p}(M)=\emptyset \neq M=H(G)$ and, therefore, $|\mathscr{K}| \stackrel{(\alpha, \beta)}{\longrightarrow}|\mathscr{K}|$ is not an $L-$ FC $^{D M R}$-morphism.

5.4.3. $L-F C^{C}$ versus $L-F^{D M R}$. The previous two subsections gave the functors $L-\mathbf{F C}_{*}^{C} \stackrel{H_{L C L D}}{\longrightarrow} L-\mathbf{F C}^{D M R}, L-\mathbf{F C}^{D M R} \stackrel{H_{L D L C}}{\longrightarrow}$ $L-\mathbf{F C}^{C}$.

Definition 98. Given an L-algebra $L, L-\mathrm{FC}_{*}^{D M R}$ is the (nonfull) subcategory of the category $L-F C^{D M R}$, with the same 
objects, whose morphisms $\mathscr{K}_{1} \stackrel{f}{\rightarrow} \mathscr{K}_{2}$ have the property that the maps $L^{G_{1}} \stackrel{\alpha}{\rightarrow} L^{G_{2}}, L^{M_{2}} \stackrel{\beta^{o p}}{\longrightarrow} L^{M_{1}}$ are surjective.

It is easy to see that there exist the restrictions $L-\mathrm{FC}_{*}^{C} \stackrel{H_{L C L D}^{*}}{\longrightarrow} L-\mathrm{FC}_{*}^{D M R}, L-\mathrm{FC}_{*}^{D M R} \stackrel{H_{L D L C}^{*}}{\longrightarrow} L-\mathrm{FC}_{*}^{C}$ of the previously mentioned two functors. A natural question could be whether the pair makes an adjoint situation. The following result, however, provides a negative answer.

Theorem 99. In general, $H_{L C L D}^{*}$ (resp., $\left.H_{L D L C}^{*}\right)$ is not a left adjoint to $H_{L D L C}^{*}$ (resp., $H_{L C L D}^{*}$ ).

Proof. Suppose that $H_{L C L D}^{*}$ is a left adjoint to $H_{L D L C}^{*}$. Then every object $\mathscr{K}$ of $L$-FC ${ }_{*}^{C}$ has an $H_{L D L C}^{*}$-universal map, that is, an $L$-FC $C_{*}^{C}$-morphism $\mathscr{K} \stackrel{\eta=(\alpha, \beta)}{\longrightarrow}\left(H_{L D L C}^{*} \circ H_{L C L D}^{*}(\mathscr{K})=\right.$ $\left.\left(L^{G}, L^{M}, \widehat{I}\right)\right)$ and, therefore, $I\left(g, \beta^{o p}(t)\right)=\widehat{I}(\alpha(g), t)$ for every $g \in G$ and every $t \in L^{M}$. It follows that $I\left(g, \beta^{o p}(t)\right) \in$ $\left\{\perp_{L}, T_{L}\right\}$. Define $G=\{g\}, M=\{m\}, L=([0,1], \bigvee, \Lambda)$ (the unit interval, with the standard lattice-theoretic structure) and $I=1 / 2$. There exists then a unique map $L^{M} \stackrel{!}{\rightarrow}$ $M$, and, moreover, one obtains that $1 / 2=I(g, !(1 / 2))=$ $\widehat{I}(\alpha(g), 1 / 2) \in\{0,1\}$, which is an obvious contradiction.

Suppose that $H_{L D L C}^{*}$ is a left adjoint to $H_{L C L D}^{*}$. Then every object $\mathscr{K}$ of $L-F_{*}^{D M R}$ has an $H_{L C L D}^{*}$-universal map, that is, an $L$-FC ${ }_{*}^{D M R}$-morphism $\mathscr{K} \stackrel{\eta=(\alpha, \beta)}{\longrightarrow}\left(H_{L C L D}^{*} \circ H_{L D L C}^{*}(\mathscr{K})=\right.$ $\left.\left(L^{G}, L^{M}, \widehat{I}\right)\right)$ and thus, $I\left(g, \beta^{o p}(t)\right)=\widehat{I}(\alpha(g), t)$ for every $g \in G$ and every $t \in L^{M}$. Similar to the aforementioned, we get a contradiction.

5.5. $L-F C^{C}$ versus $L-F_{a}^{D M R}$. The reader should notice that the results of Section 5.1 can be easily restricted to the case of the category $L-F_{a}^{D M R}$. In particular, each of Theorems 48 and 55 provides the following result.

Definition 100. Given an L-algebra $L, L-\mathbf{F C}_{*}^{C}$ is the (nonfull) subcategory of the category $L-F C^{C}$, with the same objects and with morphisms $\mathscr{K}_{1} \stackrel{f}{\rightarrow} \mathscr{K}_{2}$ such that the maps $G_{1} \stackrel{\alpha}{\rightarrow} G_{2}$, $M_{2} \stackrel{\beta^{o p}}{\longrightarrow} M_{1}$ are surjective.

Theorem 101. There exists the functor $L-\mathrm{FC}_{*}^{C} \stackrel{H_{L C L D a}}{\longrightarrow}$ $L-\mathrm{FC}_{a}^{D M R}$, which is defined by the formula $H_{L C L D a}\left(\mathscr{K}_{1} \stackrel{f}{\rightarrow}\right.$ $\left.\mathscr{K}_{2}\right)=\mathscr{K}_{1} \stackrel{\left(\alpha_{L},\left(\beta^{o p}\right)_{L}^{\leftarrow}\right)}{\longrightarrow} \mathscr{K}_{2}$.

Due to the already considerable size of the paper, the respective fixed-basis restrictions of the variable-basis setting of the previous subsections are left to the reader.

\section{Conclusion: Open Problems}

In this paper, we provided different approaches to a possible lattice-valued generalization of Formal Concept Analysis (FCA) of Ganter and Wille [31], motivated by the recent study of Denniston et al. [29] on this topic. In particular, we have constructed several categories, whose objects are latticevalued extensions of formal contexts of FCA and whose morphisms reflect the crisp setting of Pratt [13], the latticevalued setting of Denniston et al. [29], and the many-valued setting of Ganter and Wille [31] themselves. In the next step, we considered many possible functors between the newly defined categories. As a consequence, we embedded each of the constructed categories into its respective counterparts. The crucial difference of this paper from the motivating one of Denniston et al. [29] can be briefly formulated as follows.

(i) The underlying lattices of lattice-valued formal contexts are extensions of quantales, instead of restricted to precisely commutative quantales as in [29].

(ii) All the categories of lattice-valued formal contexts and almost all of their respective functors are made variable-basis (in the sense of [4]), instead of being fixed-basis as in [29].

(iii) Unlike [29], we consider the setting of many-valued formal contexts of Ganter and Wille [31].

In the wake of the constructed functors of this paper, we can single out the following important properties of the approach of Denniston et al. [29], which is based on the category of Galois connections of [32].

Firstly, their approach falls rather out of the standard settings of Pratt as well as Ganter and Wille. On one hand, there exists an obvious isomorphism between the subcategory 2-PGAL of the category GAL [32] of Galois connections on crisp powersets and the crisp category $2-\mathrm{FC}_{a}^{D M R}$ (cf. Definitions 31(1) and 37) of this paper (essentially due to Denniston et al. [29]). On the other hand, the result of Theorem 42 shows that given a unital quantale $L$, the categories $L$-PGAL and $L-\mathbf{F C}_{a}^{D M R}$ are, in general, nonisomorphic. The variablebasis approach complicates the case even more. Speaking metamathematically, while the notion of formal concept of FCA does involve an order-reversing Galois connection between the respective powersets in its definition, its main essence appears to come from a binary relation on two sets, that is, the set of objects $(G)$ and the set of their respective attributes $(M)$, which says whether a given object $g \in G$ has a given property $m \in M$. Thus, these are not the Galois connections, but their generating relations, which seem to play the main role. Moreover, Theorem 99 of the paper states that while a particular subcategory of the category of latticevalued formal contexts in the sense of Pratt is isomorphic to a nonfull subcategory of the category of Denniston et al., it does not provide a (co)reflective subcategory of the latter category with respect to the constructed functors (at the moment though, we do not know whether one can define different functors, which do give rise to a (co)reflective subcategory in question).

Secondly, the category of lattice-valued formal contexts, based on the category of Galois connections, tends to have the nature of a fixed-basis category. More precisely, while making the setting of Denniston et al. variable-basis in the current paper, we were unable to involve the morphisms of 
the underlying algebras of lattice-valued formal contexts in its definition, thereby providing a quasi-variable-basis setting.

We would like to end the section with several open problems related to the setting of this paper.

6.1. Proper Way of Building Lattice-Valued FCA. The previously mentioned discussion on the difference between the settings of binary relations and Galois connections in the lattice-valued case motivates the following open problem.

Problem 1. Is it possible to build a lattice-valued approach to FCA, which is based on order-reversing Galois connections on lattice-valued powersets, which are not generated by lattice-valued relations on their respective sets of objects and their attributes?

This problem seems especially important because we have established that using order-reversing Galois connections is more general than using relations. It may even seem that using order-reversing Galois connections with the corresponding Birkhoff operators is more robust and better than using relations. Therefore, it seems important to be able to understand how to use the Birkhoff operators when a corresponding relation may not exist. The answer may not depend on defining a relation. The answer may depend on being able to do formal concept analysis without relations.

Notice that although the notions of formal concept, protoconcept, and preconcept (cf. Definition 44) can be easily defined with the help of just a Galois connection in hand, it is their respective interpretation that poses the main problem. More precisely, what will be then in place of the crucial term "an object $g$ has an attribute $m$ "? It is our opinion that such an approach can still be developed, substituting the required single lattice-valued relation $G \times M \stackrel{I}{\rightarrow} L$ by a pair of lattice-valued relations $I_{1}(g, m)=\left(\alpha\left(\chi_{\{g\}}^{1_{L}}\right)\right)(m)$ and $I_{2}(g, m)=\left(\beta\left(\chi_{\{m\}}^{1_{L}}\right)\right)(g)$, where $L^{G} \underset{\beta}{\stackrel{\alpha}{\rightleftarrows}} L^{M}$ is a given orderreversing Galois connection, which violates the conditions of Theorem 42 (notice that the case of a commutative quantale, i.e., $\cdot \rightarrow_{l^{*}}=\cdot \rightarrow_{r}$, simplifies, but does not save the situation). In view of the discussion, a particular instance of Problem 1 is as follows.

Problem 2. What is the interpretation of the relations $I_{1}$ and $I_{2}$ and what kind of lattice-valued FCA can be developed through them?

An ad hoc approach could be to read $I_{1}(g, m)$ as the degree to which "an object $g$ has an attribute $m$ " and to read $I_{2}(g, m)$ as the degree to which "an attribute $m$ is shared by an object $g$," that is, distinguishing between the classically indistinguishable expressions "to have an attribute" and "an attribute is being shared."

6.2. Adjoint Situations between Possible Approaches to LatticeValued FCA. In the paper, we have constructed functorial embeddings of several categories of lattice-valued formal contexts into each other. However, we failed to construct a single adjoint situation between the categories in question, being able just to obtain a negative result in this respect (Theorem 99). In view of the discussion, our next problem could be formulated as follows.

Problem 3. Construct (if possible) an adjoint situation between the categories of lattice-valued formal contexts introduced in this paper.

Notice that the problem has a close relation to the discussion of the previous subsection. More precisely, an adjoint situation between the categories of lattice-valued formal contexts in the sense of Pratt and Denniston et al., respectively, can provide a partial answer to the problem of dispensing with binary relations in lattice-valued FCA and substituting them by order-reversing Galois connections.

6.3. Proper Morphisms of Lattice-Valued Formal Contexts. In the paper, we have dealt with both lattice-valued formal contexts and their respective morphisms. Whereas the former have already been treated in the fuzzy literature, the case of the latter is still not sufficiently clear. In particular, we have provided three possible approaches to (lattice-valued) formal context morphisms, that is, that of Pratt, that of Denniston et al., and that of Ganter and Wille. Taking apart the metamathematical discussion on the fruitfulness of any of the previously mentioned approaches, the last problem of the paper can be stated then as follows.

Problem 4. What is the most suitable (if any) way of defining the morphisms of lattice-valued FCA?

All the previously mentioned open problems will be addressed in our subsequent articles on the topic of latticevalued FCA. An interested reader is kindly invited to share the effort.

\section{Acknowledgments}

The author is grateful to Professor W. Tholen and Professor J. Steprans, who invited him to spend the first six months of the year 2012 at the Department of Mathematics and Statistics of York University (Toronto, ON, Canada), during which period the paper was prepared. The author would also like to thank J. T. Denniston, A. Melton (Kent State University), and S. E. Rodabaugh (Youngstown State University) for going through the draft version of the paper, pointing out its various deficiencies and suggesting their adjustments. This research was supported by the ESF Project no. CZ.1.07/2.3.00/20.0051 "Algebraic Methods in Quantum Logic" of the Masaryk University in Brno, Czech Republic. S of $\mathbf{L}, \mathbf{S}-\mathbf{F C}_{a}^{D M R}$ following

\section{References}

[1] S. A. Solovyov, "Variable-basis topological systems versus variable-basis topological spaces," Soft Computing, vol. 14, no. 10, pp. 1059-1068, 2010.

[2] S. Vickers, Topology via Logic, Cambridge University Press, Cambridge, UK, 1989. 
[3] J. T. Denniston and S. E. Rodabaugh, "Functorial relationships between lattice-valued topology and topological systems," Quaestiones Mathematicae, vol. 32, no. 2, pp. 139-186, 2009.

[4] S. E. Rodabaugh, "Categorical foundations of variable-basis fuzzy topology," in Mathematics of Fuzzy Sets: Logic, Topology and Measure Theory, U. Höhle and S. E. Rodabaugh, Eds., vol. 3 of The Handbooks of Fuzzy Sets Series, pp. 273-388, Kluwer Academic Publishers, Dordrecht, The Netherlands, 1999.

[5] C. Guido, "Attachment between fuzzy points and fuzzy sets," in Abstracts of the 30th Linz Seminar on Fuzzy Set Theory, U. Bodenhofer, B. De Baets, E. P. Klement, and S. Saminger-Platz, Eds., pp. 52-54, Johannes Kepler Universität, Linz, Austria, 2009.

[6] C. Guido, "Fuzzy points and attachment," Fuzzy Sets and Systems, vol. 161, no. 16, pp. 2150-2165, 2010.

[7] U. Höhle, "A note on the hypergraph functor," Fuzzy Sets and Systems, vol. 131, no. 3, pp. 353-356, 2002.

[8] J. T. Denniston, A. Melton, and S. E. Rodabaugh, "Lattice-valued topological systems," in Abstracts of the 30th Linz Seminar on Fuzzy Set Theory, U. Bodenhofer, B. De Baets, E. P. Klement, and S. Saminger-Platz, Eds., pp. 24-31, Johannes Kepler Universität, Linz, Austria, 2009.

[9] J. T. Denniston, A. Melton, and S. E. Rodabaugh, "Interweaving algebra and topology: Lattice-valued topological systems," Fuzzy Sets and Systems, vol. 192, pp. 58-103, 2012.

[10] T. Kubiak and A. Šostak, "Foundations of the theory of $(L, M)$ fuzzy topological spaces," in Abstracts of the 30th Linz Seminar on Fuzzy Set Theory, U. Bodenhofer, B. De Baets, E. P. Klement, and S. Saminger-Platz, Eds., pp. 70-73, Johannes Kepler Universität, Linz, Austria, 2009.

[11] J. T. Denniston, A. Melton, and S. E. Rodabaugh, "Lattice-valued predicate transformers and interchange systems," in Abstracts of the 31st Linz Seminar on Fuzzy Set Theory, P. Cintula, E. P. Klement, and L. N. Stout, Eds., pp. 31-40, Johannes Kepler Universität, Linz, Austria, 2010.

[12] E. W. Dijkstra, A Discipline of Programming, Prentice-Hall, Englewood, Ill, USA, 1976.

[13] V. Pratt, "Chu spaces," in School on Category Theory and Applications. Lecture Notes of Courses, Coimbra, Portugal, vol. 21 of Textos Matemáticas Series B, pp. 39-100, Universidade de Coimbra, Departamento de Matemática, Coimbra, Portugal, 1999.

[14] M. Barr, *-Autonomous Categories. With An Appendix By PoHsiang Chu, Springer-Verlag, New York, NY, USA, 1979.

[15] J. T. Denniston, A. Melton, and S. E. Rodabaugh, "Latticevalued predicate transformers, Chu games, and lattice-valued transformer systems," Fuzzy Sets and Systems. In press.

[16] D. Aerts, "Foundations of quantum physics: a general realistic and operational approach," International Journal of Theoretical Physics, vol. 38, no. 1, pp. 289-358, 1999.

[17] D. Aerts, E. Colebunders, A. Van Der Voorde, and B. Van Steirteghem, "State property systems and closure spaces: a study of categorical equivalence," International Journal of Theoretical Physics, vol. 38, no. 1, pp. 359-385, 1999.

[18] D. Aerts, E. Colebunders, A. Van Der Voorde, and B. Van Steirteghem, "On the amnestic modification of the category of state property systems," Applied Categorical Structures, vol. 10, no. 5, pp. 469-480, 2002.

[19] S. A. Solovyov, "On a generalization of the concept of state property system," Soft Computing, pp. 1-12, 2011.
[20] S. A. Solovyov, "Hypergraph functor and attachment," Fuzzy Sets and Systems, vol. 161, no. 22, pp. 2945-2961, 2010.

[21] S. A. Solovyov, "Categorical foundations of variety-based topology and topological systems," Fuzzy Sets and Systems, vol. 192, pp. 176-200, 2012.

[22] S. A. Solovyov, "Localification of variable-basis topological systems," Quaestiones Mathematicae, vol. 34, no. 1, pp. 11-33, 2011.

[23] J. C. Kelly, "Bitopological spaces," Proceedings London Mathematical Society, vol. 13, no. 3, pp. 71-89, 1963.

[24] C. J. Mulvey and J. W. Pelletier, "On the quantisation of points," Journal of Pure and Applied Algebra, vol. 159, no. 2-3, pp. 231295, 2001.

[25] C. J. Mulvey and J. W. Pelletier, "On the quantisation of spaces," Journal of Pure and Applied Algebra, vol. 175, no. 1-3, pp. 289325, 2002.

[26] S. Solovyov, "Composite variety-based topological theories," Fuzzy Sets and Systems, vol. 195, pp. 1-32, 2012.

[27] S. A. Solovyov, "Generalized fuzzy topology versus noncommutative topology," Fuzzy Sets and Systems, vol. 173, no. 1, pp. 100-115, 2011.

[28] S. Solovyov, "Lattice-valuedsoft algebras," Soft Computing. In press.

[29] J. T. Denniston, A. Melton, and S. E. Rodabaugh, "Formal concept analysis and lattice-valued Chu systems," Fuzzy Sets and Systems, vol. 216, pp. 52-90, 2013.

[30] J. T. Denniston, A. Melton, and S. E. Rodabaugh, "Formal concept analysis and lattice-valued interchange systems," in Abstracts of the 32nd Linz Seminar on Fuzzy Set Theory, D. Dubois, M. Grabisch, R. Mesiar, and E. P. Klement, Eds., pp. 41-47, Johannes Kepler Universität, Linz, Austria, 2011.

[31] B. Ganter and R. Wille, Formale Begriffsanalyse. Mathematische Grundlagen, Springer, Berlin, Germany, 1996.

[32] J. M. McDill, A. C. Melton, and G. E. Strecker, "A category of Galois connections," in Proceedings of the Conference on Category Theory and Computer Science, vol. 283 of Lecture Notes in Computer Science, pp. 290-300, Edinburgh, UK, 1987.

[33] R. B. Bêlohlávek, Fuzzy Relational Systems. Foundations and Principles, Kluwer Academic Publishers, New York, NY, USA, 2002.

[34] D. Kruml and J. Paseka, "Algebraic and categorical aspects of quantales," Handbook of Algebra, vol. 5, pp. 323-362, 2008.

[35] K. I. Rosenthal, Quantales and Their Applications, vol. 234 of Pitman Research Notes in Mathematics, Addison Wesley Longman, Boston, Mass, USA, 1990.

[36] B. Vormbrock and R. Wille, "Semiconcept and protoconcept algebras: the basic theorems," in Formal Concept Analysis. Foundations and Applications, B. Ganter, G. Stumme, and R. Wille, Eds., vol. 3626 of Lecture Notes in Computer Science, pp. 34-48, Springer, Berlin, Germany, 2005.

[37] M. Erné, J. Koslowski, A. Melton, and G. E. Strecker, "A primer on Galois connections," Annals of the New York Academy of Sciences, vol. 704, pp. 103-125, 1993.

[38] R. Bêlohlávek, "Fuzzy Galois connections," Mathematical Logic Quarterly, vol. 45, no. 4, pp. 497-504, 1999.

[39] G. Birkhoff, Lattice Theory, American Mathematical Society Publications, New York, NY, USA, 1940.

[40] J. G. García, I. Mardones-Pérez, M. A. de Prada Vicente, and D. Zhang, "Fuzzy Galois connections categorically," Mathematical Logic Quarterly, vol. 56, no. 2, pp. 131-147, 2010. 
[41] J. Adámek, H. Herrlich, and G. E. Strecker, Abstract and Concrete Categories: The Joy of Cats, Dover Publications, Mineola, NY, USA, 2009.

[42] H. Herrlich and G. E. Strecker, Category Theory, vol. 1 of Sigma Series in Pure Mathematics, Heldermann Verlag, Berlin, Germany, 3rd edition, 2007.

[43] S. Mac Lane, Categories For the Working Mathematician, Springer-Verlag, New York, NY, USA, 2nd edition, 1998.

[44] P. M. Cohn, Universal Algebra, D. Reidel Publishing Company, Dordrecht, The Netherlands, 1981.

[45] G. Richter, Kategorielle Algebra, Akademie-Verlag, Berlin, Germany, 1979.

[46] B. Banaschewski and E. Nelson, "Tensor products and bimorphisms," Canadian Mathematical Bulletin, vol. 19, no. 4, pp. 385402, 1976.

[47] E. G. Manes, Algebraic Theories, Springer-Verlag, New York, NY, USA, 1976.

[48] P. T. Johnstone, Stone Spaces, Cambridge University Press, Cambridge, UK, 1982.

[49] S. E. Rodabaugh, "Relationship of algebraic theories to powerset theories and fuzzy topological theories for Lattice-valued mathematics," International Journal of Mathematics and Mathematical Sciences, vol. 2007, Article ID 43645, 71 pages, 2007.

[50] G. Gierz, K. H. Hofmann, K. Keimel et al., Continuous Lattices and Domains, Cambridge University Press, Cambridge, UK, 2003.

[51] S. E. Rodabaugh, "Powerset operator based foundation for point-set lattice-theoretic (poslat) fuzzy set theories and topologies," Quaestiones Mathematicae, vol. 20, no. 3, pp. 463-530, 1997.

[52] S. E. . Rodabaugh, "Powerset operator foundations for poslat fuzzy set theories and topologies," in Mathematics of Fuzzy Sets: Logic, Topology and Measure Theory, U. Höhle and S. E. Rodabaugh, Eds., vol. 3 of The Handbooks of Fuzzy Sets Series, pp. 91-116, Kluwer Academic Publishers, Dordrecht, The Netherlands, 1999.

[53] S. E. Rodabaugh, "Relationship of algebraic theories to powersets over objects in Set and Set× C," Fuzzy Sets and Systems, vol. 161, no. 3, pp. 453-470, 2010.

[54] S. Solovyov, "Powerset operator foundations for catalg fuzzy set theories," Iranian Journal of Fuzzy Systems, vol. 8, no. 2, pp. 1-46, 2011.

[55] S. Krajči, "A categorical view at generalized concept lattices," Kybernetika, vol. 43, no. 2, pp. 255-264, 2007.

[56] G. Q. Zhang, "Chu spaces, concept lattices, and domains," in Proceedings of the 9th Conference on the Mathematical Foundations of Programming Semantics (Montreal '03), vol. 83 of Electronic Notes in Theoretical Computer Science, 2004.

[57] K. Deiters and M. Erné, "Sums, products and negations of contexts and complete lattices," Algebra Universalis, vol. 60, no. 4, pp. 469-496, 2009.

[58] M. Erné, Categories of Contexts, University of Hannover, Hanover, Germany, 1990.

[59] B. Ganter, "Relational Galois connections," in Proceedings of the 5th International Conference on Formal Concept Analysis, S. O. Kuznetsov, Ed., vol. 4390 of Lecture Notes in Computer Science, pp. 1-17, 2007.

[60] H. Mori, "Functorial properties of formal concept analysis," in Proceedings of the 15th International Conference on Conceptual Structures, U. Priss, Ed., vol. 4604 of Lecture Notes in Computer Science, pp. 505-508, 2007.
[61] L. Shen and D. Zhang, "The concept lattice functors," International Journal of Approximate Reasoning, vol. 54, no. 1, pp. 166183, 2013.

[62] A. Frascella, "Fuzzy Galois connections under weak conditions," Fuzzy Sets and Systems, vol. 172, no. 1, pp. 33-50, 2011.

[63] M. E. Della Stella and C. Guido, "L-relations and Galois triangles," in Algebraic Semantics for Uncertainty and Vagueness, Palazzo Genovese, Salerno, Italy, 2011.

[64] A. Frascella and C. Guido, "Transporting many-valued sets along many-valued relations," Fuzzy Sets and Systems, vol. 159, no. 1, pp. 1-22, 2008. 


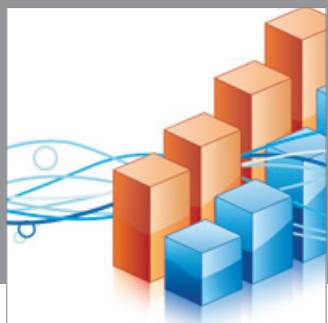

Advances in

Operations Research

mansans

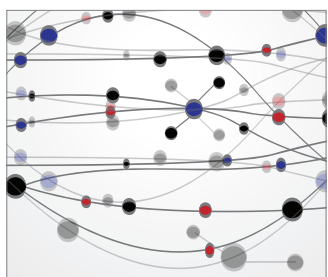

The Scientific World Journal
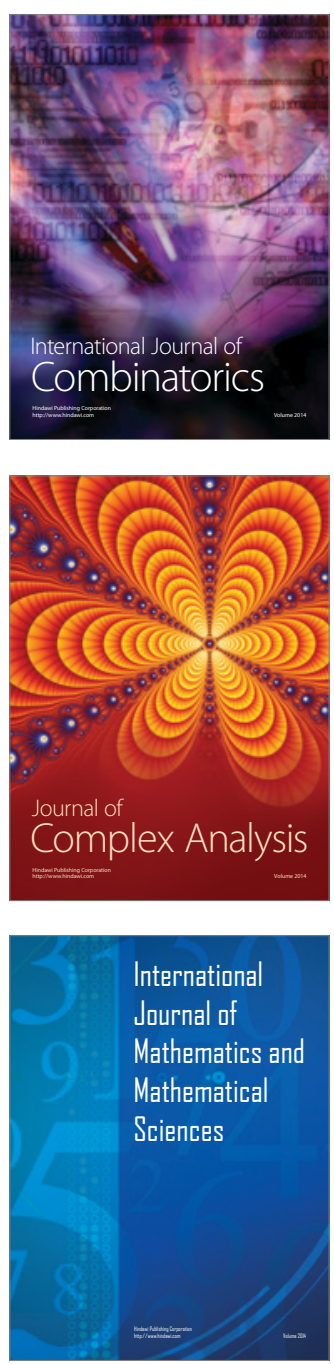
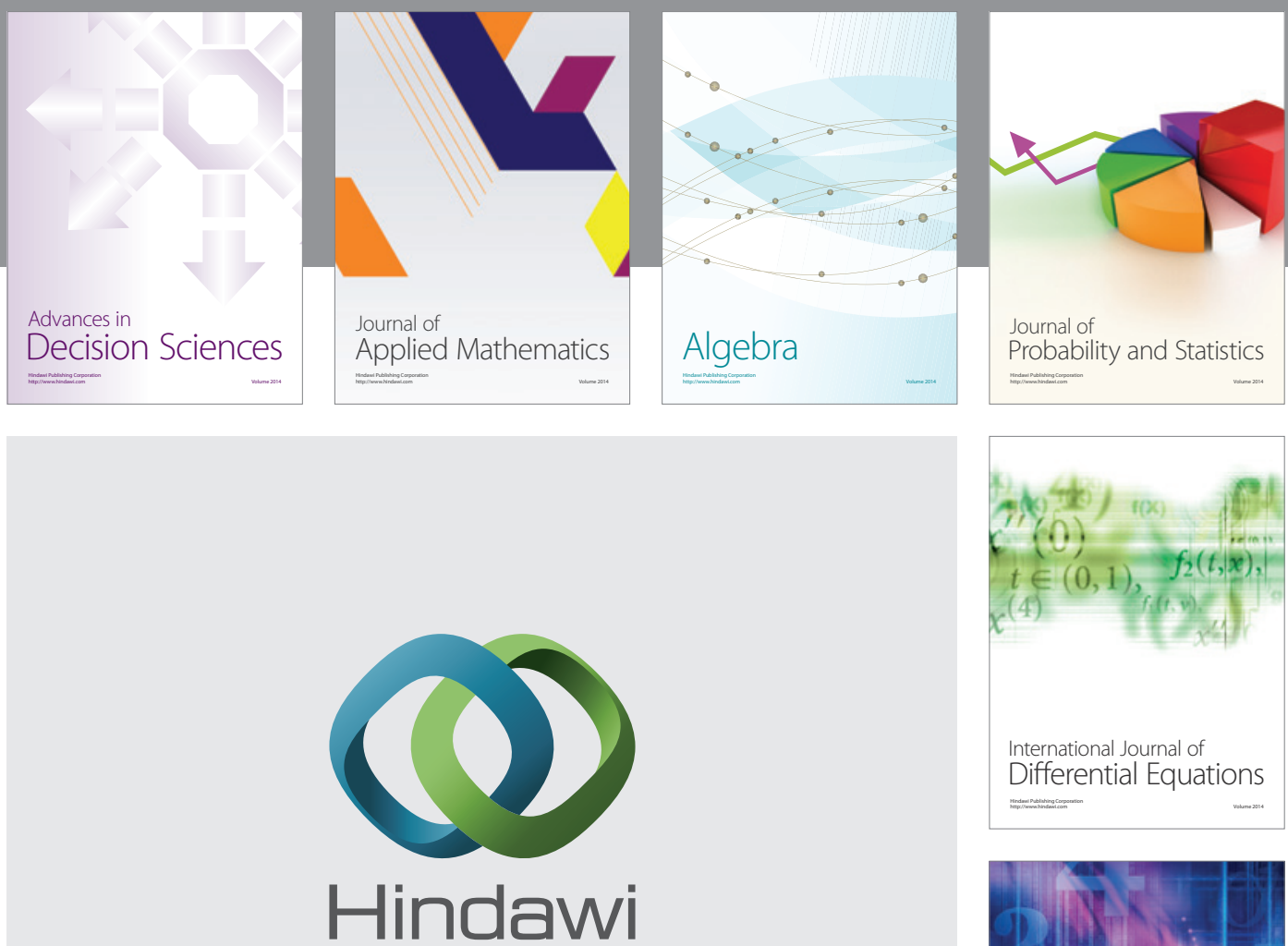

Submit your manuscripts at http://www.hindawi.com
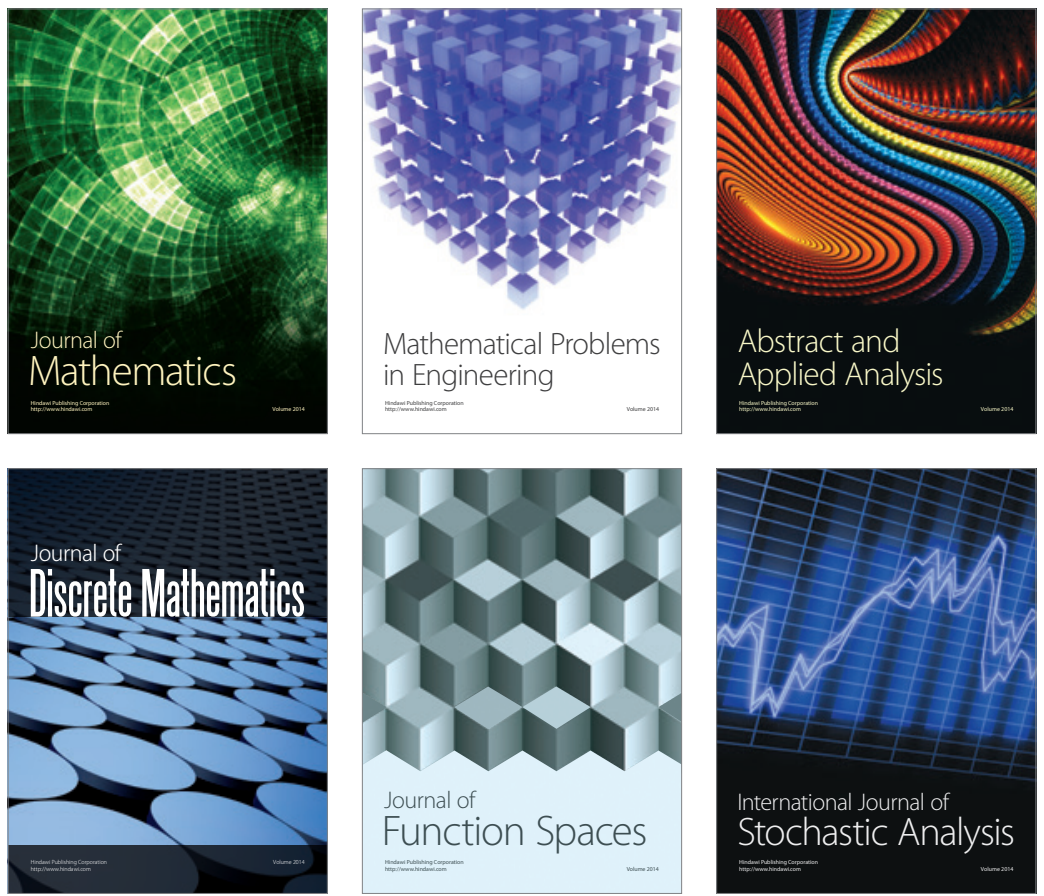

Journal of

Function Spaces

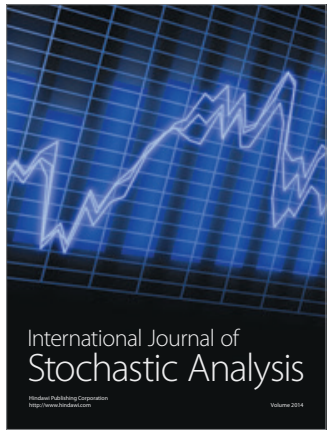

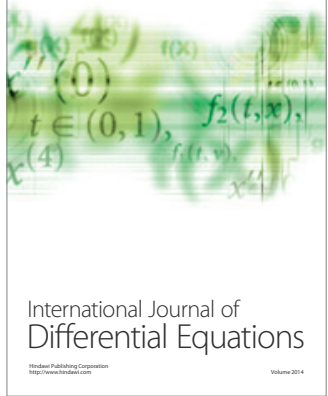
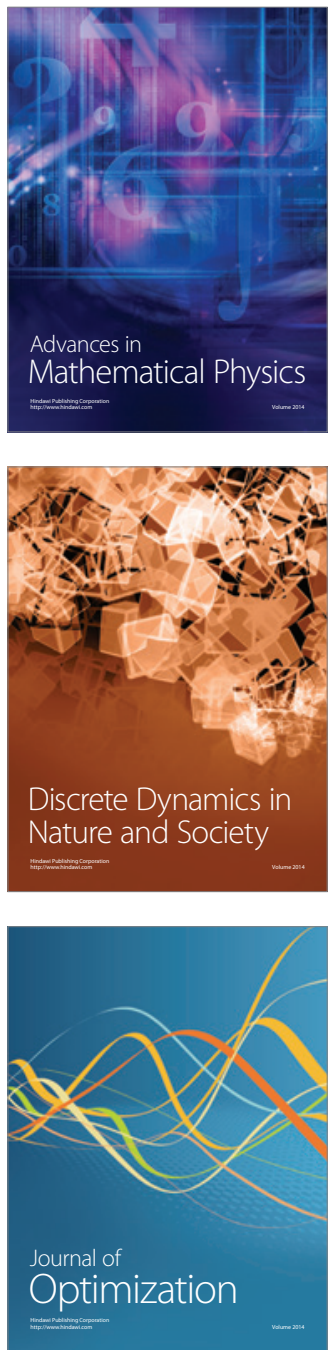